\title{
Charged complexity and the thermofield double state
}

\author{
Shira Chapman ${ }^{a, b}$ and Hong Zhe (Vincent) Chen ${ }^{c}$ \\ ${ }^{a}$ Department of Physics, Ben-Gurion University of the Negev, \\ Beer Sheva 84105, Israel \\ ${ }^{b}$ Institute for Theoretical Physics, University of Amsterdam, \\ Science Park 904, Postbus 94485, 1090 GL Amsterdam, The Netherlands \\ ${ }^{c}$ Perimeter Institute for Theoretical Physics, \\ 31 Caroline Street North, Waterloo, ON N2L $2 Y 5$, Canada \\ E-mail: schapman@bgu.ac.il, hchen2@perimeterinstitute.ca
}

ABSTRACT: We establish a systematic framework for studying quantum computational complexity of Gaussian states of charged systems based on Nielsen's geometric approach. We use this framework to examine the effect of a chemical potential on the dynamics of complexity. As an example, we consider the complexity of a charged thermofield double state constructed from two free massive complex scalar fields in the presence of a chemical potential. We show that this state factorizes between positively and negatively charged modes and demonstrate that this fact can be used to relate it, for each momentum mode separately, to two uncharged thermofield double states with shifted temperatures and times. We evaluate the complexity of formation for the charged thermofield double state, both numerically and in certain analytic expansions. We further present numerical results for the time dependence of complexity. We compare various aspects of these results to those obtained in holography for charged black holes.

KEywords: AdS-CFT Correspondence, Holography and condensed matter physics (AdS/CMT), Conformal Field Theory

ArXiv EPRINT: 1910.07508 


\section{Contents}

1 Introduction 1

2 Charged complexity from complex covariance matrices $\quad 6$

3 Complex scalar field theory $\quad 11$

$\begin{array}{lll}3.1 & \text { Preliminaries } & 12\end{array}$

\begin{tabular}{ll}
3.2 & Normal mode decomposition on the lattice \\
\hline
\end{tabular}

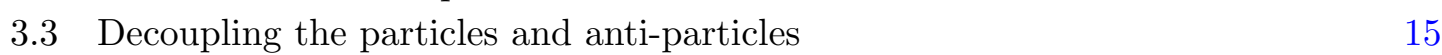

$\begin{array}{ll}3.4 & \text { The reference state } \\ \end{array}$

4 Complexity of the cTFD state $\quad 17$

$\begin{array}{lll}\text { 4.1 Properties of the uncharged thermofield double state } & 18\end{array}$

$\begin{array}{lll}4.2 & \text { cTFD of two complex harmonic oscillators } & 19\end{array}$

4.3 Complexity of the cTFD of two complex harmonic oscillators 21

4.4 cTFD states of the complex scalar 24

$\begin{array}{ll}4.5 & \text { Complexity of formation }\end{array}$

$\begin{array}{ll}\text { 4.5.1 Vanishing chemical potential } & 27\end{array}$

$\begin{array}{ll}\text { 4.5.2 High temperature limit } & 27\end{array}$

$\begin{array}{lll}\text { 4.5.3 Low temperature limit } & 28\end{array}$

$\begin{array}{lll}\text { 4.5.4 Numerical results } & 28\end{array}$

4.6 Time dependence 32

5 Comparison with holographic results 36

$\begin{array}{lll}6 & \text { Summary and outlook } & 40\end{array}$

A Basis of $\mathfrak{s p}(2 N, \mathbb{R})$ for real/complex phase space operators 41

B Time evolution of the cTFD state 44

C Derivation of the low temperature limits $\quad 46$

D Diagonalization of the relative covariance matrix at $t=0 \quad 49$ 


\section{Introduction}

Quantum computational complexity provides an estimate of the difficulty of constructing quantum states for the purpose of performing quantum computations [1,2]. Traditionally, complexity is defined for spin/qubit chains, using a universal set of unitary gates chosen such that each gate acts only on a small number of spins/qubits. Appropriately chosen sequences of these gates, i.e., circuits, are able to reproduce, to a given precision, arbitrary target states of the spin/qubit chain, starting from a simple unentangled reference state. The complexity of a given target state is then defined as the minimal number of gates required for such a circuit. The problem of finding the shortest circuits is challenging if one naively attempts an exhaustive check of all possible circuits. An alternative geometric approach, proposed by Nielsen, translates the problem of finding the minimal circuits to a geometric problem of finding geodesics in the manifold of unitaries equipped with the metric that naturally arises from the algebra on its tangent space [3-5].

Nielsen's approach was extended to Gaussian states of quantum field theories (QFTs) in [6], where the authors studied the complexity of the vacuum state of a free scalar QFT (see [7] for an alternative approach based on the Fubini-Study (FS) metric). Extensions for various classes of Gaussian states were also studied, see, e.g., [8-15]. Despite this progress, little is known beyond the free field theory regime, see however some recent progress in [16-19].

In holography, quantum complexity is proposed to be related to certain geometric bulk observables by means of the complexity=volume (CV) proposal $[20,21]$, relating the complexity to the volume of a maximal bulk slice anchored at the boundary time slice where the state is defined, and the complexity=action $(\mathrm{CA})$ proposal $[22,23]$, relating the complexity to the action of a certain region in the bulk - the Wheeler-DeWitt (WDW) patch bounded by light sheets and anchored at that same boundary time slice. Complexity comes about as part of a wider line of research studying the way in which quantum information notions are encoded in gravity, which dates back to the relation between entanglement entropy and Ryu-Takayanagi surfaces, see, e.g., [24, 25].

The properties of the complexity of Gaussian states of free QFTs turn out to have surprising qualitative similarities to the properties of holographic complexity. ${ }^{1}$ This is despite the fact that these free systems are significantly simpler than the strongly coupled theories which are of interest in holography. One similarity has to do with the structure of divergences in complexity [6, 7]. Complexity in QFT is divergent due to the necessity of establishing short range correlations in the QFT state. This is similar to what happens for entanglement entropy. The divergences have to be regulated by the introduction of a UV cutoff, for example, a lattice spacing $\delta$. It turns out that the structure of divergences of complexity is similar when comparing the free QFT results with those found in holography, see $[6,7,26,27]$ and the discussion of [28], with a leading divergence of the form of a volume law. For subregions of the vacuum state, one obtains in the complexity, in addition to the leading volume law divergence, also a subleading area law divergence proportional to the

\footnotetext{
${ }^{1}$ As is standard in the literature, we use "holographic complexity" here and elsewhere to mean the bulk quantities (volume or action) dual to the CFT complexity in the CV and CA conjectures.
} 
entanglement entropy, both in holography and QFT [15]. It is expected that the qualitative comparison of free field theory results to those obtained from gravity will provide hints toward a good definition of complexity on the field theory side applicable to the dual field theory of holographic systems.

Despite being very well studied on the holographic side of the correspondence (as we will explain below), the complexity of charged systems received very little attention on the quantum field theory side and in particular no models for complexity of Gaussian states in charged systems have been examined. The goal of this work is to fill this gap by establishing a systematic computational framework for evaluating the complexity in charged systems and for studying the effect of chemical potential on the dynamics of complexity in these systems. Our formalism utilizes complex phase space operators which turn out to provide a simpler description of Gaussian states of charged systems. We will use our newly developed formalism to study the complexity of the Gaussian charged version of the thermofield double (TFD) state constructed from two copies of a free complex scalar QFT.

This "charged thermofield double state" (cTFD) is a particularly symmetric purification of a mixed state in the grand canonical ensemble at finite temperature and chemical potential. The purification is obtained by constructing a pure state on two identical copies of the system on which the mixed state is defined and is given by the following expression, see e.g., $[29]^{2}$

$$
\left|\operatorname{cTFD}\left(t_{L}, t_{R}\right)\right\rangle=\frac{1}{\sqrt{Z_{\beta, \mu}}} \sum_{n, \sigma} e^{-\beta\left(E_{n}+\mu c_{\sigma}\right) / 2-i\left(E_{n}+\mu c_{\sigma}\right)\left(t_{L}+t_{R}\right)}\left|E_{n}, c_{\sigma}\right\rangle_{L}\left|E_{n},-c_{\sigma}\right\rangle_{R} .
$$

In the above expression, the two copies of the system are denote L (the "left" copy) and $\mathrm{R}$ (the "right" copy) and are both equipped with an identical Hamiltonian. $t_{L, R}$ are the times on the left and right copies, $\beta=1 / T$ is the inverse temperature, $\mu$ is the chemical potential and $\left|E_{n}, c_{\sigma}\right\rangle$ are a basis of energy and charge eigenstates where $E_{n}$ is the energy eigenvalue and $c_{\sigma}$ is the eigenstate of a conserved global $\mathrm{U}(1)$ charge. Finally, $Z_{\beta, \mu}$ is a normalization constant. The two states in each term were chosen to have opposite charges such that they are CPT conjugates [29]. It is apparent from this expression that the time evolution of the cTFD is governed by the deformed Hamiltonians $H_{L, R} \pm \mu C_{R, L}$, where $C_{R, L}$ are the $\mathrm{U}(1)$ charges on the left/right copies, respectively. The neutral thermofield double (TFD) state is simply obtained in the $\mu=0$ limit of the above expression.

TFD and cTFD states received special attention in holographic studies due to their duality to (neutral and charged) eternal black holes of the corresponding temperature [30] and chemical potential [31, 32]. Outside the context of holography, a number of works have been written on building the TFD state in the laboratory [33-37] and these further motivate focusing on the TFD for simple quantum mechanical systems. Since charge is very natural in an experimental setup, we expect that the cTFD state is also a natural state to study in the laboratory.

\footnotetext{
${ }^{2}$ We have traded the traditional symbol $Q$ for the charge in favour of $C$, in anticipation of using $Q$ as the position operator for the harmonic oscillators in the Nielsen construction. Consequently, we will use $c$ to denote eigenvalues of charge.
} 
The complexity of formation of the cTFD and TFD states [23, 38, 39] is defined as the additional complexity required in order to prepare the entangled (c)TFD state compared to preparing both copies of the field theory in their vacuum state. This quantity is UV finite. For the uncharged TFD state, it was found that the complexity of formation is proportional to the entropy with positive proportionality coefficient, both in free QFT and in holography ${ }^{3}[11,38]$. This provides another point of similarity between holography and free field theory. The complexity of formation in charged black hole backgrounds was also studied in holography in $[23,39]$ and was found to diverge in the extremal (zerotemperature) limit. This is a result of an IR divergence due to the infinitely long throat of the wormhole in the extremal limit. The interpretation suggested in [39] was that of a third law of complexity, namely that cTFD states at finite chemical potential and zero temperature are infinitely more complex compared to their finite temperature counterparts and cannot be formed by any physical process in a finite amount of time. ${ }^{4}$ We will use our formalism for charged complexity to verify the third law of complexity for simple Gaussian states and check how universal it is.

The time dependence of complexity of the TFD and cTFD states using the two holographic proposals was also studied. It was found that the complexity increases (approximately) linearly as a function of the time $t_{L}=t_{R}=t / 2$, for a very long time, much longer than the typical time it takes for other observables, e.g., the entanglement entropy [40], to saturate. In fact, it was suggested that holographic complexity keeps growing for an exponential amount of time, until the semi-classical gravity approximation breaks down [23]. This unusual time dependence captures the growth of the volume of the wormhole/EinsteinRosen bridge connecting the two sides of the geometry via the behind horizon region [41] and is also typical of complexity in spin chains of fast scrambling systems, e.g., [42, 43]. ${ }^{5}$ The linear growth of complexity (for long times) served as one of the original motivations for proposing the holographic conjectures for complexity (CV and CA). Additional evidence for the validity of these conjectures follows from the effects of perturbations on the complexity, and in particular from the manifestation of chaos and scrambling [21, 28, 42, 47, 48]. The simple Gaussian states in free QFTs fail to capture the properties of complexity typical to fast scrambling systems such as the time dependence of complexity and its reaction to perturbations.

For charged black holes, the rate of change in complexity over time was found to vanish in the extremal (vanishing temperature) limit at all times using both the CV and the CA complexity proposals [39]. It is interesting to verify how generic this property is - for

\footnotetext{
${ }^{3}$ The holographic result is valid for planar black holes in $d>2$ using both CV and CA, where $d$ is the number of boundary spacetime dimensions.

${ }^{4}$ It is worth mentioning that the gravitational setup with charged black holes is somewhat advantageous compared to the neutral setup especially with regards to evaluating the complexity with the CA proposal since this result is influenced by regions of the WDW patch which go arbitrarily near the singularity of the neutral black hole. This does not happen for charged black holes due to the different causal structure including two horizons.

${ }^{5}$ Fast scramblers are systems which spread the effects of localized perturbations in a time which is logarithmic in the entropy [44]. Holographic systems are expected to be fast scrambling [45, 46].
} 
example, we would like to understand whether this property would hold also for simpler Gaussian cTFD states in charged systems.

To test the effect of charge on properties of the complexity in a simple setup and as a specific example to our charged complexity formalism, we explore the complexity of Gaussian cTFD states constructed from two copies of a free complex scalar QFT. ${ }^{6}$ Our motivation to focus on the free cTFD state is twofold. First, we would like to qualitatively compare its complexity to the holographic results. Second, since charges are of experimental relevance in various quantum mechanical systems, we expect that those results will come to use also in the context of quantum information physics.

The complex scalar field factorizes to a set of complex harmonic oscillators with frequencies $\omega_{k}=\sqrt{k^{2}+m^{2}}$ where $m$ is the mass of the complex scalar and $k$ is the spatial momentum. Furthermore, each harmonic oscillator can be decomposed using particle and anti-particle modes. A very simple relation then connects the energy and charge to the number operators for the particle and anti-particle modes, i.e., the energy is proportional to the sum of the numbers of particles and anti-particles and the charge to their difference. It then becomes obvious that we can regard the cTFD state of each mode as a product of two independent TFD states - one which includes particles on the left copy and antiparticles on the right copy and another one which includes particles on the right copy and anti-particles on the left copy. These two TFD states are associated with effective shifted temperatures and times given $b^{7}$

$$
\beta_{k, \mathrm{eff}} \equiv \beta\left(1 \pm \frac{\mu}{\omega_{k}}\right), \quad t_{k, \mathrm{eff}} \equiv t\left(1 \pm \frac{\mu}{\omega_{k}}\right)
$$

respectively, see eq. (4.22)-(4.23), where $\omega_{k}$ are the frequencies of the oscillators. Note that we have a different effective temperature and time for each mode. It is worth emphasizing that while the cTFD state (for each momentum mode) factorizes to two uncharged TFD states, the complexity of the cTFD state cannot be directly related to the complexity of the two uncharged TFD states, see footnote 17. The effective temperatures in eq. (1.2) imply that the cTFD state is only well defined so long as $|\mu|<\omega_{k}$. As $\mu \rightarrow \omega_{k}$, one of the effective temperatures approaches infinity and this means that the associated mode will be

\footnotetext{
${ }^{6}$ A previous attempt to study the complexity of cTFDs was made in [49] using the Fubini-Study approach. However, in that work, the authors found that the complexity grows linearly for a long time for the free complex scalar QFT. We find that this is a gross overestimate of complexity since they have not identified correctly the optimal circuit. This is easy to see - while their control function in eq. (38) starts and stops at the right points, it periodically tracks back on itself over the course of the circuit when $t$ is large. Considering the $k$-th mode for concreteness, when $\left(\omega_{k}+\mu q\right) t$ is a multiple of $2 \pi$, the reference and target states coincide for that mode (see their eqs. (12) and (37)), so its complexity contribution should vanish eq. (38) fails this basic test as is clear from the non-vanishing integrand in the third line of (A-1) when $t$ is a multiple of $\frac{2 \pi}{\omega_{k}+\mu q}$. Hence the circuit trajectory is clearly not the optimal one.

${ }^{7}$ We have set the elementary unit of charge to one in eq. (1.2). This amounts to the replacement $\mu \leftrightarrow \mu c$ where $c$ is the elementary unit of charge in our results below.
} 
infinitely populated. This forces us to consider only ${ }^{8}|\mu|<m$ and prevents us from taking the conformal limit $m \rightarrow 0$ while holding the chemical potential fixed when studying the complexity of the full fledged complex scalar QFT. It would be interesting to verify if this problem can be avoided in a different setup. ${ }^{9}$ Furthermore, it would be interesting to include the condensate explicitly in evaluating the complexity. We leave these problems for the future.

In evaluating the complexity of the charged thermofield double state we find that the third law of complexity is not reproduced in the free charged scalar example. We do find however that the computation slows down as we decrease the temperature which is similar to what happens in holography.

This paper is organized as follows. In section 2, we present our framework for studying charged complexity in the context of quantum mechanical systems. We explain how to generalize Nielsen's complexity approach to circuits between Gaussian states using covariance matrices defined with respect to complex phase space operators which are useful in the presence of charge. In section 3, we review the various necessary ingredients for studying the complexity of states in a QFT of a complex scalar. In section 4, we explain how to evaluate the complexity of the cTFD state. We present our numerical results for the complexity of formation, as well as a number of analytic expansions for low and high temperatures. We then present numerical results for the time dependence of the complexity of the cTFD state. In section 5, we compare our results to those obtained in holography. We end with a summary and outlook in section 6 . We have left a number of technical details for appendices. In appendix A, we present the generators used to evaluate the complexity as explained in section 2. In appendix B, we present an explanation for the form of the time evolution of the cTFD state. Finally, in appendices $\mathrm{C}$ and $\mathrm{D}$, we present additional details required for evaluating the low temperature limits in section 4.5.3.

\footnotetext{
${ }^{8}$ In any dimension, taking $|\mu|>m$ leads to a non-normalizable state as all modes in the range $0 \leq|k| \leq$ $\sqrt{\mu^{2}-m^{2}}$ become infinitely populated. In $d \leq 2$, just taking the limit $|\mu| \rightarrow m$ leads to a non-normalizable state, as can be seen from the divergence of the (vacuum-subtracted) grand canonical potential (which is proportional to the logarithm of the normalization factor). In $d \geq 3$, when naively neglecting possible condensation in the zero mode $k=0$, the $|\mu| \rightarrow m$ state is normalizable, but perhaps still physically questionable at $d=3$ as it gives an infinite particle number density. Finally, in $d \geq 4$, states with $|\mu|=m$ correspond to a phase with Bose-Einstein condensation where the zero mode $k=0$ makes up a nontrivial fraction of the total (finite) particle number density. (The derivation of these facts follows from a straightforward extension of the standard statistical mechanics treatment of a Bose gas, except here we consider the relativistic spectrum $\omega_{k}=\sqrt{k^{2}+m^{2}}$ rather than the non-relativistic spectrum $\frac{k^{2}}{2 m}$.) In this paper, however, whenever we take the $|\mu| \rightarrow m$ limit, we shall assume that the contribution from the condensate is negligible. We expect this to be the case, for example, when the $|\mu| \rightarrow m$ limit and the limit of infinite spatial extent $L$ are taken such that we are on the boundary of (or just inside the) uncondensed phase, where the number density $\frac{1}{L^{d-1}} \frac{1}{e^{\beta(m-|\mu|)}-1}$ of the condensate vanishes.

${ }^{9}$ For example, by studying the theory in a (sufficiently small) finite volume with Dirichlet boundary conditions, one should be able to take the $m \rightarrow 0$ limit without encountering a condensate.
} 


\section{Charged complexity from complex covariance matrices}

We start by generalizing several aspects of Nielsen's geometric approach to the quantum complexity of Gaussian states using covariance matrices [11] to account for the possibility of complex phase space operators. Complex phase space operators are a simple rotation of real phase space operators as we demonstrate below, but as we will see, they provide a much more natural framework for studying charged systems. In particular, they are very commonly used when studying charged (complex) scalar field theory.

Gaussian states are completely characterized by the one and two-point functions of a set of phase space operators. When working with complex phase space operators, we start by listing the coordinates and their conjugates as follows ${ }^{10}$

$$
\xi_{\mathbb{C}}=\left(q_{1}, q_{1}^{\dagger}, \ldots, q_{N / 2}, q_{N / 2}^{\dagger}, p_{1}^{\dagger}, p_{1}, \ldots, p_{N / 2}^{\dagger}, p_{N / 2}\right)
$$

where we have specifically chosen to invert the order of the momentum operators $p_{i}^{\dagger}$ and $p_{i}$ compared to the order of the corresponding position operators $q_{i}$ and $q_{i}^{\dagger}$ and where $N$, the number of (real) degrees of freedom, is an even integer. The complex phase space operators can also be re-expressed in terms of real phase space operators

$$
\xi_{\mathbb{R}}=\left(q_{1}, \ldots, q_{N}, p_{1}, \ldots, p_{N}\right)
$$

where here, $N$ is the same (even integer) as in eq. (2.1). From now on we will use subscripts $\mathbb{C}$ and $\mathbb{R}$ to refer to the cases of complex and real phase space operators, respectively. Statements written without a subscript are correct for both types of operators. Note that $\xi$ and $\xi^{\dagger}$ are not independent from each other but are rather related according to

$$
\left(\xi^{a}\right)^{\dagger}=A^{a}{ }_{b} \xi^{b}
$$

where $A$ is the following matrix

$$
A_{\mathbb{R}}=\mathbb{1}_{2 N \times 2 N}, \quad A_{\mathbb{C}}=\bigoplus_{I=1 \ldots N}\left[\begin{array}{ll}
0 & 1 \\
1 & 0
\end{array}\right],
$$

for the cases of real and complex operators, respectively. $\bigoplus$ stands for a direct sum of 2 by 2 matrices acting on each pair of operators by their order of appearance in eq. (2.1). Note that both these transformations satisfy $A^{2}=1$ and $A^{\mathrm{T}}=A$.

The phase space operators satisfy canonical commutation relations given in terms of the symplectic form $\Omega$ as follows ${ }^{11}$

$$
\left[\xi^{a},\left(\xi^{b}\right)^{\dagger}\right]=i \Omega^{a b}, \quad \Omega=\left[\begin{array}{cc}
0 & \mathbb{1}_{N \times N} \\
-\mathbb{1}_{N \times N} & 0
\end{array}\right] .
$$

\footnotetext{
${ }^{10}$ Although we do not put hats on top of our phase space operators, here we always mean operators in the quantum mechanical system.

${ }^{11}$ In the first equality of eq. (2.5), $\Omega^{a b}$ is implicitly multiplying a unit operator in the Hilbert space.
} 
As mentioned earlier, we will focus on Gaussian states which are completely characterized by the two point functions of the phase space operators

$$
\left\langle\psi\left|\xi^{a}\left(\xi^{b}\right)^{\dagger}\right| \psi\right\rangle \equiv \frac{1}{2}\left(G^{a b}+i \Omega^{a b}\right) .
$$

In the above expression, the anti-symmetric part is the matrix $\Omega^{a b}$, and the symmetric part

$$
G^{a b}=G^{(a b)}=\left\langle\psi\left|\xi^{a}\left(\xi^{b}\right)^{\dagger}+\left(\xi^{b}\right)^{\dagger} \xi^{a}\right| \psi\right\rangle
$$

is known as the covariance matrix. Note that the matrix representation of the covariance matrix, i.e., $G$, is Hermitian, and therefore satisfies $\left(G^{\dagger}\right)^{a b}=\left(G^{b a}\right)^{\dagger}=G^{a b}$. Everywhere in this paper, we will only focus on Gaussian states with vanishing one point functions $\left\langle\psi\left|\xi^{a}\right| \psi\right\rangle=0$ which will be completely characterized by their covariance matrices. By restricting our attention to Gaussian states, i.e., selecting a reference and target state which are Gaussian and only considering circuits moving through the space of Gaussian states, we are able to make some progress in solving for the optimal circuits in the complexity geometry. The treatment of complexity using covariance matrices was proposed in [11] as an alternative to the wavefunction approach of [6]. This approach proves simpler in cases where the circuit utilizes gates which are quadratic in both the position and the momentum operators such as when constructing the TFD and cTFD states.

The action of a circuit moving between Gaussian states can be parameterized by the action of Hermitian generators which are quadratic in the canonical operators $\xi$,

$$
\begin{gathered}
|\tilde{\psi}\rangle=\hat{U}|\psi\rangle, \quad \hat{U}=e^{-i \hat{K}}, \\
\hat{K} \equiv \frac{1}{2}\left(\xi^{a}\right)^{\dagger} k_{a b} \xi^{b},
\end{gathered}
$$

where $k_{a b}$ is the matrix characterization of a given Hermitian generator $\hat{K}$. Note that in order for $\hat{K}$ to be Hermitian, the matrix $k_{a b}$ should satisfy

$$
k=k^{\dagger} \text {. }
$$

Furthermore, due to the relation (2.3), we can assume, without loss of generality, that $A \cdot k$ is symmetric, namely that the $k$ matrices satisfy

$$
k^{\mathrm{T}}=A \cdot k \cdot A .
$$

The action of the unitary in eq. (2.8) on the state can be represented directly as an operation on the covariance matrix. To see this, we start by exploring the effect of conjugating the canonical operators with the unitary operation (2.8)

$$
\tilde{\xi}^{a} \equiv \hat{U}^{\dagger} \xi^{a} \hat{U}=\sum_{n=0}^{\infty} \frac{1}{n !}\left[i \hat{K}, \xi^{a}\right]_{(n)}
$$

where the Taylor expansion is given in terms of the nested commutator defined recursively by $\left[i \hat{K}, \xi^{a}\right]_{(n)} \equiv\left[i \hat{K},\left[i \hat{K}, \xi^{a}\right]_{(n-1)}\right]$, where $\left[i \hat{K}, \xi^{a}\right]_{(0)}=\xi^{a}$. Using the commutation relations in eq. (2.5), we find

$$
\left[i \hat{K}, \xi^{a}\right]=\frac{1}{2}\left[\Omega \cdot\left(A \cdot k^{T} \cdot A+k\right)\right]_{b}^{a} \xi^{b}=(\Omega \cdot k)_{b}^{a} \xi^{b} \equiv K_{b}^{a} \xi^{b},
$$


where we have defined

$$
K \equiv \Omega \cdot k, \quad K^{\dagger}=-k \cdot \Omega .
$$

Resumming eq. (2.12), we obtain

$$
\tilde{\xi}^{a}=S^{a}{ }_{b} \xi^{b}, \quad S \equiv e^{K} .
$$

It is then straightforward to check that this induces the following transformations on the covariance matrix $(2.7)$

$$
\tilde{G}=S \cdot G \cdot S^{\dagger}
$$

where $\tilde{G}$ is the covariance matrix associated with the state $|\tilde{\psi}\rangle$. The unitary conjugation (2.12) does not modify the commutation relations since

$$
\left[\tilde{\xi}^{a}, \tilde{\xi}^{b}\right]=S(i \Omega) S^{\dagger}=(i \Omega)
$$

which is satisfied automatically by virtue of the identity $K \Omega+\Omega K^{\dagger}=0$, see eq. (2.14). Note however that this last condition is less restrictive than requiring $K=\Omega \cdot k$ for some $k$ satisfying (2.10)-(2.11).

The next step is to identify a basis of independent generators which span the space of possible Hermitian generators $\hat{K}$ in eq. (2.9). Naively, we could think that taking a basis for general Hermitian matrices $k_{a b}$ in eq. (2.9) would naturally induce a basis for the independent Hermitian generators. However, due to the relation (2.3), this would be over-counting and it is straightforward to see that the independent generators are instead given by matrices $k$ which satisfy the conditions (2.10)-(2.11).

Whether the operators are real or complex, the number of independent generators for these transformations between Gaussian states can be counted by counting the number of constraints in eqs. (2.10)-(2.11) and is equal to $N(2 N+1)$. This is due to the fact that the group of all $S$ in eq. (2.15), where $K=\Omega \cdot k$ and $k$ satisfies the conditions (2.10)-(2.11), is simply isomorphic to $\operatorname{Sp}(2 N, \mathbb{R})=\left\{e^{\bar{K}} \in M_{2 N \times 2 N}(\mathbb{R})\right.$ with $\left.\bar{K} \cdot \Omega+\Omega \cdot \bar{K}^{T}=0\right\}$. To see this, first note that the algebra of generators of $\operatorname{Sp}(2 N, \mathbb{R})$ can be recast as the algebra of real symmetric matrices $\bar{k}$ defined by $\bar{K}=\Omega \cdot \bar{k}$, since the condition that $\bar{k}$ be symmetric is satisfied if and only if $\bar{K} \cdot \Omega+\Omega \cdot \bar{K}^{T}=0$. Further note that we may then relate the generators $\bar{k}$ to the independent generators $k$ satisfying eqs. (2.10)-(2.11) according to $\bar{k}=k$ for real phase space operators and $\bar{k}=R \cdot k \cdot R^{\dagger}$, where

$$
R=\bigoplus_{I=1 \ldots N} \frac{1}{\sqrt{2}}\left[\begin{array}{cc}
1 & 1 \\
-i & i
\end{array}\right],
$$

for complex phase space operators, and these $\bar{k}$ will automatically be real and symmetric using eqs. (2.10)-(2.11) due to the identity $A_{\mathbb{C}} \cdot R^{\mathrm{T}}=R^{\dagger}$.

We will label by $K_{I}$ a complete basis of independent generators for the transformations above and will generally assume that they are orthonormal with respect to the inner product

$$
\frac{1}{2} \operatorname{tr}\left(K_{I} K_{J}^{\dagger}\right)=\delta_{I J}
$$


Explicit expressions for those generators using both real and complex operators can be found in appendix A.

At the next step, we will want to construct circuits through the space of Gaussian states. The circuits will act on the covariance matrices while leaving the symplectic form invariant, i.e.,

$$
G(\sigma)=S(\sigma) G_{\mathrm{ref}} S^{\dagger}(\sigma), \quad S(\sigma) \Omega S^{\dagger}(\sigma)=\Omega,
$$

where $\sigma \in[0,1]$ is a trajectory parameter. Boundary conditions are imposed such that the circuit $G(\sigma)$ moves between the covariance matrix of the reference state $G_{\text {ref }}$ at $\sigma=0$ and ends at the covariance matrix of the target state $G_{\text {target }}$ at $\sigma=1$. Explicitly, this means that $S(\sigma=0)$ acts trivially on $G_{\text {ref }}$, while

$$
S(\sigma=1) G_{\text {ref }} S^{\dagger}(\sigma=1)=G_{\text {target }} .
$$

The symplectic transformation $S(\sigma)$ can be decomposed according to

$$
S(\sigma) \equiv \overleftarrow{\mathcal{P}} \exp \int_{0}^{\sigma} d \sigma^{\prime} Y^{I}\left(\sigma^{\prime}\right) K_{I}
$$

where $K_{I}$ are the generators of the symplectic group, assumed to be orthonormal, as in eq. (2.19), and $\overleftarrow{\mathcal{P}}$ denotes right to left path ordering, i.e., the product integral of infinitesimal exponentials re-ordered from right to left in ascending order in $\sigma^{\prime}$. Eq. (2.22) may be interpreted as decomposing the path $S(\sigma)$ through the space of unitary operations on Gaussian states as a sequence of infinitesimal operations generated by $K_{I}$ turned on by the control functions $Y^{I}(\sigma)$. This is completely analogous to the usual quantum computation picture mentioned in section 1 , where a circuit is composed of a sequential application of gates chosen from some set of elementary gates - here $S(\sigma)$ is the circuit and infinitesimal exponentiation of the generators $K_{I}$ produces the elementary gates (or rather, their action on the covariance matrices).

This geometric approach was implemented in [11] using several different cost functions $F$ to evaluate the length

$$
d[S(\sigma)]=\int_{0}^{1} d \sigma F\left(S(\sigma), Y^{I}(\sigma)\right)
$$

of a given circuit. The cost functions considered were:

$$
F_{1}=\sum_{I}\left|Y^{I}\right|, \quad F_{2}=\sqrt{\sum_{I}\left(Y^{I}\right)^{2}}, \quad D_{\kappa}=\sum_{I}\left|Y^{I}\right|^{\kappa} .
$$

The complexity of the target state is given by the length of the shortest circuit, i.e., path $S(\sigma)$ through $\operatorname{Sp}(2 N, \mathbb{R})$, satisfying the boundary condition (2.21) (as well as acting trivially on the reference state at $\sigma=0)$, for a given choice of cost function,

$$
\mathcal{C} \equiv \min _{S(\sigma)} d[S(\sigma)]
$$


Note that the result for the complexity is basis dependent, i.e., in general the choice of basis $K_{I}$ influences this result. However, the $F_{2}$ and $\kappa=2$ cost functions remain unchanged when the two bases are related by an orthogonal transformation on the position operators and an identical orthogonal transformation on the momentum operators.

Before proceeding, let us comment on the dimensions of various quantities introduced thus far. In order for Nielsen's notion of complexity, defined in eqs. (2.23)-(2.25), to be sensible generalizations of gate counting, it must be dimensionless. This is achieved by considering dimensionless phase space operators $\xi$ (and hence dimensionless $F, Y^{I}$, $K_{I}, K$, and $k$ ). In order to absorb the dimensions intrinsic to the usual phase space operators of physical systems, it will therefore be necessary to introduce a dimensionful scale $\omega_{g}$, see e.g., section 2.2 .3 of [11]. In section 3.2, we shall use $\omega_{g}$ to translate between dimensionful physical operators and the dimensionless operators with respect to which complexity is defined.

A particularly simple circuit between the reference state and the target state is the 'straight line' circuit, obtained by exponentiating a constant Lie algebra element multiplied by $\sigma$. The straight line circuit can be obtained as follows. Given a target state covariance matrix $G_{\text {target }}$ and a reference state covariance matrix $G_{\text {ref }}$, the relative covariance matrix is defined to be their ratio:

$$
\Delta_{\text {target }} \equiv G_{\text {target }} G_{\text {ref }}^{-1}
$$

The straight line circuit is then given by

$$
S(\sigma)=e^{\sigma K}, \quad \text { where } \quad K=\frac{1}{2} \log \Delta_{\text {target }} .
$$

It was proved in [11] that, for the case of the $F_{2}$ and $D_{\kappa=2}$ cost functions, when the reference state scale and the gate scale (to be introduced below in section 3.2) are taken to be equal, the path of minimal cost is indeed the straight line circuit. With these scales equal to each other, it was further suggested in [11] that the straight line circuit provides a good approximation for the $F_{1}$ complexity. In other cases, [11] have suggested that the straight line circuit yields a non-trivial upper bound for the complexity.

Of the cost functions considered in [11], it was found that the $F_{1}$ cost produces results most similar to those obtained in holography using the holographic complexity proposals, for the structure of UV divergences in the complexity of the full boundary state [6] as well as that of mixed states [15]. Hence, in this note, we too will focus on the $F_{1}$ complexity, evaluating it on the straight line circuit. Furthermore, since the $F_{1}$ cost function depends on the choice of generators, two choices were proposed in [11]. The first choice retains the left-right coordinate split between the two sides of the TFD, while the second choice mixes the two into a "diagonal" basis. The first choice was found to yield properties more similar to those of holographic complexity, in particular in reproducing the proportionality between the complexity of formation and the entropy. We will therefore focus on this choice in this paper.

In order to obtain the $F_{1}$ complexity, we have to decompose the trajectory $(2.27)$ according to the expression (2.22) and extract the scalar coefficients $Y^{I}$, which appear in 
the cost function. Since we have assumed that our basis of generators $K_{I}$ is orthonormal, see eq. (2.19), we can do this by using the inner product

$$
Y^{I}=\frac{1}{2} \operatorname{tr}\left(K K_{I}^{\dagger}\right) \text {. }
$$

Finally, an upper bound $\mathcal{C}_{1}^{\mathrm{UB}}$ for the complexity $\mathcal{C}_{1}$ associated with the $F_{1}$ cost function is given by integrating $F_{1}$ along the straight line circuit. Since the generator of the trajectory is simply constant along the path, this yields

$$
\mathcal{C}_{1} \leq \mathcal{C}_{1}^{\mathrm{UB}}=\sum_{I}\left|Y^{I}\right|=\frac{1}{2} \sum_{I}\left|\operatorname{tr}\left(K K_{I}^{\dagger}\right)\right|=\frac{1}{4} \sum_{I}\left|\operatorname{tr}\left(\log \left(\Delta_{\text {target }}\right) \cdot K_{I}^{\dagger}\right)\right| .
$$

Before we end this section, let us mention that, in order to move between different bases of generators, it is possible to use a coordinate transformation

$$
\tilde{\xi}^{a}=R_{b}^{a} \xi^{b}
$$

where $R$ is a general complex matrix preserving the commutation relations

$$
\Omega=R \cdot \Omega \cdot R^{\dagger} .
$$

The covariance matrices, circuit (2.20) and generators (2.15) get rotated according to

$$
\tilde{G}=R \cdot G \cdot R^{\dagger}, \quad \tilde{S}=R \cdot S \cdot R^{-1}, \quad \tilde{K}=R \cdot K \cdot R^{-1} .
$$

One such useful transformation mentioned earlier is the one moving between the real and complex operators, i.e., $\xi_{\mathbb{R}}=R_{\mathbb{C} \rightarrow \mathbb{R}} \xi_{\mathbb{C}}$ where $R_{\mathbb{C} \rightarrow \mathbb{R}}$ is the same transformation as given in eq. (2.18). Note that not all such basis transformations can be represented as unitary transformations acting on the state $|\psi\rangle$ used to define the covariance matrix. Finally, let us point out that the inner product (2.28) has to be evaluated in the basis in which we want to compute the complexity. Alternatively, we may compute it in a different basis by using the rotated inner product

$$
Y^{I}=\frac{1}{2} \operatorname{tr}\left(\tilde{K} \tilde{G}_{\mathbb{1}} \tilde{K}_{I}^{\dagger} \tilde{G}_{\mathbb{1}}^{-1}\right),
$$

defined in terms of the positive symmetric matrix $\tilde{G}_{\mathbb{1}}=R R^{\dagger}$, where $R$ is the transformation matrix between the two bases.

\section{Complex scalar field theory}

In this section, we establish our notation for the complex scalar QFT and explain how to put it on a lattice. This translates the problem of studying the complexity of charged states in this QFT to a problem of studying the complexity of charged states of a set of coupled harmonic oscillators, which can then be addressed using the tools of section 2 . In subsection 3.3, we present a useful choice of basis which decouples the contributions of the particles and anti-particles to the Hamiltonian and charge. This basis is generally useful for studying the complexity of charged states, which are typically defined in terms of eigenstates of these two operators. We will demonstrate how to use all this machinery to study the specific example of the complexity of the cTFD state in the next section. 


\subsection{Preliminaries}

We will focus on a theory consisting of a complex scalar field in $d$ spacetime dimensions. The Hamiltonian of the system is given in terms of the fields $\phi, \phi^{\dagger}$ and conjugate momenta $\pi, \pi^{\dagger}$ according to

$$
H=\int d^{d-1} x\left(\pi^{\dagger} \pi+\vec{\nabla} \phi^{\dagger} \cdot \vec{\nabla} \phi+m^{2} \phi^{\dagger} \phi\right)
$$

The field and momentum operators obey the equal time commutation relations $[\phi(\vec{x}), \pi(\vec{y})]=\left[\phi^{\dagger}(\vec{x}), \pi^{\dagger}(\vec{y})\right]=i \delta^{d-1}(\vec{x}-\vec{y})$. The charge is given by

$$
C=i \int d^{d-1} x\left(\phi^{\dagger} \pi^{\dagger}-\phi \pi\right)
$$

In the above expression, we chose our convention such that the fundamental unit of charge is set to one, but of course, this dependence can be recovered later by redefining the chemical potential appropriately. The complex scalar field can be decomposed in terms of the following Fourier modes

$$
\begin{aligned}
& \phi(x)=\int \frac{d^{d-1} p}{(2 \pi)^{d-1}} \frac{1}{\sqrt{2 \omega_{p}}}\left(a_{\vec{p}} e^{-i \omega_{p} t+i \vec{p} \cdot \vec{x}}+\bar{a}_{\vec{p}}^{\dagger} e^{i \omega_{p} t-i \vec{p} \cdot \vec{x}}\right), \\
& \pi(x)=-i \int \frac{d^{d-1} p}{(2 \pi)^{d-1}} \sqrt{\frac{\omega_{p}}{2}}\left(\bar{a}_{\vec{p}} e^{-i \omega_{p} t+i \vec{p} \cdot \vec{x}}-a_{\vec{p}}^{\dagger} e^{i \omega_{p} t-i \vec{p} \cdot \vec{x}}\right),
\end{aligned}
$$

where $a_{\vec{p}}, a_{\vec{p}}^{\dagger}$ and $\bar{a}_{\vec{p}}, \bar{a}_{\vec{p}}^{\dagger}$ are annihilation and creation operators for particle and antiparticle modes, respectively, satisfying the following commutation relations

$$
\left[a_{\vec{p}}, a_{\vec{p}^{\prime}}^{\dagger}\right]=\left[\bar{a}_{\vec{p}}, \bar{a}_{\vec{p}^{\prime}}^{\dagger}\right]=(2 \pi)^{d-1} \delta\left(\vec{p}-\vec{p}^{\prime}\right),
$$

and where $\omega_{p} \equiv \sqrt{\vec{p}^{2}+m^{2}}$. In terms of those creation and annihilation operators, the Hamiltonian and charge are given by

$$
H=\int \frac{d^{d-1} p}{(2 \pi)^{d-1}} \omega_{p}\left(a_{\vec{p}}^{\dagger} a_{\vec{p}}+\bar{a}_{\vec{p}} \bar{a}_{\vec{p}}^{\dagger}\right), \quad C=\int \frac{d^{d-1} p}{(2 \pi)^{d-1}}\left(a_{\vec{p}}^{\dagger} a_{\vec{p}}-\bar{a}_{\vec{p}}^{\dagger} \bar{a}_{\vec{p}}\right) .
$$

This reflects the fact that particles and antiparticles contribute to the energy of a given state according to the sum of their number operators while contributing to the charge with opposite signs.

\subsection{Normal mode decomposition on the lattice}

As explained in section 1, the complexity is divergent and has to be regularized. This was done in $[6,11]$ by placing the theory on a spatial periodic lattice. Hence, we will start by briefly reviewing how to place the free complex scalar on such a lattice. The resulting theory will be a sum of harmonic oscillators for the different momentum modes.

We will use a periodic lattice of size $L$ in each space direction with $N^{d-1}$ sites and lattice spacing $\delta=L / N$. For convenience, we assume that $N$ is odd. The different sites will be labelled by indices

$$
\vec{a} \equiv\left(a_{1}, \ldots, a_{d-1}\right) \in\{-\tilde{N}, \ldots, \tilde{N}\}^{d-1}, \quad \text { where } \quad \tilde{N} \equiv \frac{N-1}{2} .
$$


The discretized versions of eqs. (3.1) and (3.2) take the form

$$
\begin{aligned}
& H=\sum_{\vec{a}}\left[\delta \hat{P}_{\vec{a}}^{\dagger} \hat{P}_{\vec{a}}+m^{2} \delta^{-1} \hat{Q}_{\vec{a}}^{\dagger} \hat{Q}_{\vec{a}}+\delta^{-3} \sum_{j}\left(\hat{Q}_{\vec{a}+\vec{e}_{j}}-\hat{Q}_{\vec{a}}\right)^{\dagger}\left(\hat{Q}_{\vec{a}+\vec{e}_{j}}-\hat{Q}_{\vec{a}}\right)\right], \\
& C=i \sum_{\vec{a}}\left(\hat{Q}_{\vec{a}}^{\dagger} \hat{P}_{\vec{a}}^{\dagger}-\hat{Q}_{\vec{a}} \hat{P}_{\vec{a}}\right),
\end{aligned}
$$

where we have defined

$$
\hat{Q}_{\vec{a}} \equiv \delta^{d / 2} \phi(\delta \cdot \vec{a}), \quad \hat{P}_{\vec{a}} \equiv \delta^{d / 2-1} \pi(\delta \cdot \vec{a}),
$$

$\vec{e}_{j}$ denotes the unit vector in the $j$-th direction and we have written the position and momentum operators with hats in order to keep the symbols $P, Q$ free for later use. These coordinates and momentum operators satisfy the commutation relations

$$
\left[\hat{Q}_{\vec{a}}, \hat{P}_{\vec{b}}\right]=i \delta_{\vec{a}, \vec{b}}
$$

We see that, on the lattice, the field theory reduces to a theory of coupled harmonic oscillators. To decouple these oscillators, we move into Fourier space by defining

$$
\begin{aligned}
\tilde{Q}_{\vec{n}} & \equiv N^{-\frac{d-1}{2}} \sum_{\vec{a}} e^{-\frac{2 \pi i \vec{n} \cdot \vec{a}}{N}} \hat{Q}_{\vec{a}}, \quad \tilde{P}_{\vec{n}} \equiv N^{-\frac{d-1}{2}} \sum_{\vec{a}} e^{\frac{2 \pi i \vec{n} \cdot \vec{a}}{N}} \hat{P}_{\vec{a}}, \\
n & \equiv\left(n_{1}, \ldots, n_{d-1}\right) \in\{-\tilde{N}, \ldots, \tilde{N}\}^{d-1} .
\end{aligned}
$$

Note that we have chosen opposite signs for the phases in the Fourier transforms of the position and momentum operators. These operators satisfy the commutation relations

$$
\left[\tilde{Q}_{\vec{n}}, \tilde{P}_{\vec{k}}\right]=i \delta_{\vec{n}, \vec{k}}
$$

The Hamiltonian and charge then read ${ }^{12}$

$$
\begin{aligned}
& H=\sum_{\vec{n}}\left(\delta \tilde{P}_{\vec{n}}^{\dagger} \tilde{P}_{\vec{n}}+\omega_{n}^{2} \delta^{-1} \tilde{Q}_{\vec{n}}^{\dagger} \tilde{Q}_{\vec{n}}\right), \quad \omega_{n}^{2} \equiv m^{2}+\frac{4}{\delta^{2}} \sum_{j} \sin ^{2}\left(\frac{n_{j} \pi}{N}\right), \\
& C=i \sum_{\vec{n}}\left(\tilde{Q}_{\vec{n}}^{\dagger} \tilde{P}_{\vec{n}}^{\dagger}-\tilde{Q}_{\vec{n}} \tilde{P}_{\vec{n}}\right) .
\end{aligned}
$$

In order to gain physical intuition, it is also instructive to consider the decomposition of the scalar field in terms of creation and annihilation operators. On the lattice, the complex scalar field and its conjugate momentum have mode expansions ${ }^{13}$

$$
\begin{aligned}
& \phi(\delta \cdot \vec{a}, t)=L^{-\frac{d-1}{2}} \sum_{\vec{n}} \frac{1}{\sqrt{2 \omega_{n}}}\left(a_{\vec{n}} e^{-i\left(\omega_{n} t-\frac{2 \pi \vec{n} \cdot \vec{a}}{N}\right)}+\bar{a}_{\vec{n}}^{\dagger} e^{i\left(\omega_{n} t-\frac{2 \pi \vec{n} \cdot \vec{a}}{N}\right)}\right), \\
& \pi(\delta \cdot \vec{a}, t)=-i L^{-\frac{d-1}{2}} \sum_{\vec{n}} \sqrt{\frac{\omega_{n}}{2}}\left(\bar{a}_{\vec{n}} e^{-i\left(\omega_{n} t-\frac{2 \pi \vec{n} \cdot \vec{a}}{N}\right)}-a_{\vec{n}}^{\dagger} e^{i\left(\omega_{n} t-\frac{2 \pi \vec{n} \cdot \vec{a}}{N}\right)}\right),
\end{aligned}
$$

\footnotetext{
${ }^{12}$ Throughout the following, we will stick to the convention where $\tilde{Q}_{\vec{n}}^{\dagger}$ is the complex conjugate of $\tilde{Q}_{\vec{n}}$, rather than being the Fourier transform of the coordinate $Q_{\vec{a}}^{\dagger}$, with conventions as in (3.10).

${ }^{13}$ The creation and annihilation operators in this section are dimensionless and are related to the ones in the previous section according to $a_{\vec{p}}^{\text {continuous }}=L^{\frac{d-1}{2}} a_{\vec{n}}^{\text {lattice }}$ where $\vec{p}=\frac{2 \pi}{L} \vec{n}$.
} 
where $\vec{a}, \vec{n}$ take values as indicated by eqs. (3.6) and (3.10), $\omega_{n}$ is defined in eq. (3.12), and

$$
\left[a_{\vec{n}}, a_{\vec{n}^{\prime}}^{\dagger}\right]=\left[\bar{a}_{\vec{n}}, \bar{a}_{\vec{n}^{\prime}}^{\dagger}\right]=\delta_{\vec{n} \vec{n}^{\prime}}
$$

with other creation and annihilation commutators vanishing. Using eqs. (3.13), (3.10) and (3.8) we can deduce

$$
\tilde{Q}_{\vec{n}}=\sqrt{\frac{\delta}{2 \omega_{n}}}\left(a_{\vec{n}}+\bar{a}_{-\vec{n}}^{\dagger}\right), \quad \tilde{P}_{\vec{n}}=-i \sqrt{\frac{\omega_{n}}{2 \delta}}\left(\bar{a}_{-\vec{n}}-a_{\vec{n}}^{\dagger}\right) .
$$

The $a_{\vec{n}}, \bar{a}_{\vec{n}}$ can be regarded as annihilation operators for particles and anti-particles respectively since the Hamiltonian and charge of the field are given by

$$
H=\sum_{\vec{n}} \omega_{n}\left(N_{\vec{n}}+\bar{N}_{\vec{n}}+1\right), \quad C=\sum_{\vec{n}}\left(N_{\vec{n}}-\bar{N}_{\vec{n}}\right),
$$

where the number operators are defined to be

$$
N_{\vec{n}} \equiv a_{\vec{n}}^{\dagger} a_{\vec{n}}, \quad \bar{N}_{\vec{n}} \equiv \bar{a}_{\vec{n}}^{\dagger} \bar{a}_{\vec{n}}
$$

Note that one usually normal-orders the Hamiltonian, removing the last term of the summand in the first equation of (3.16) representing the zero point energy.

As already mentioned in the discussion below eq. (2.25), the gates used in constructing quantum circuits for studying complexity in this paper and also those used to construct the TFD state in [11] consist of quadratic combinations of the coordinate and momentum operators. Since these are dimensionful operators we will have to introduce an additional scale $\omega_{g}$ (with inverse length dimensions) in our complexity model. This scale is used to rescale the position and momentum operators in such a way that they become dimensionless

$$
\tilde{q}_{\vec{n}} \equiv \omega_{g} \tilde{Q}_{\vec{n}}=\sqrt{\frac{1}{2 \lambda_{n}}}\left(a_{\vec{n}}+\bar{a}_{-\vec{n}}^{\dagger}\right), \quad \tilde{p}_{\vec{n}} \equiv \frac{\tilde{P}_{\vec{n}}}{\omega_{g}}=-i \sqrt{\frac{\lambda_{n}}{2}}\left(\bar{a}_{-\vec{n}}-a_{\vec{n}}^{\dagger}\right)
$$

where we have defined

$$
\lambda_{n} \equiv \frac{\omega_{n}}{\mu_{g}}, \quad \text { where } \quad \mu_{g} \equiv \delta \omega_{g}^{2},
$$

and we refer to $\mu_{g}$ as the gate scale.

Using these new dimensionless operators, we can express the Hamiltonian and charge in eq. (3.12) as

$$
H=\sum_{\vec{n}} \omega_{n}\left(\lambda_{n}^{-1} \tilde{p}_{\vec{n}}^{\dagger} \tilde{p}_{\vec{n}}+\lambda_{n} \tilde{q}_{\vec{n}}^{\dagger} \tilde{q}_{\vec{n}}\right), \quad C=i \sum_{\vec{n}}\left(\tilde{q}_{\vec{n}}^{\dagger} \tilde{p}_{\vec{n}}^{\dagger}-\tilde{q}_{\vec{n}} \tilde{p}_{\vec{n}}\right) .
$$

For later reference, we denote this basis of complex operators by:

$$
\xi_{\vec{n}}^{\mathbb{C}}=\left[\tilde{q}_{\vec{n}}, \tilde{q}_{\vec{n}}^{\dagger}, \tilde{p}_{\vec{n}}^{\dagger}, \tilde{p}_{\vec{n}}\right]^{\mathrm{T}} .
$$

Recall from eq. (2.5) that the unusual ordering of the operators and their conjugates was chosen such that $\left[\xi_{\vec{n}}^{\mathbb{C}},\left(\xi_{\vec{n}}^{\mathbb{C}}\right)^{\dagger}\right]=i \Omega$. 


\subsection{Decoupling the particles and anti-particles}

So far, we have reframed the theory of the complex scalar field as a theory of decoupled harmonic oscillators in the complex "C્C" basis defined in eq. (3.21). Since many states in this field theory are defined in terms of eigenvalues of the Hamiltonian and charge, it will be useful to perform an additional transformation to identify degrees of freedom associated with positive and negative charges. This transformation decouples the contributions of particles and anti-particles to the Hamiltonian and charge operators simultaneously. This transformation is given by the explicit expression

$$
\begin{aligned}
\xi_{\vec{n}}^{\mathbb{R}} \equiv R_{\mathbb{C} \rightarrow \mathbb{R}} \xi_{\vec{n}}^{\mathbb{n}}, \quad \xi_{\vec{n}}^{\mathbb{C}} \equiv\left[\begin{array}{c}
\tilde{q}_{\vec{n}} \\
\tilde{q}_{\vec{n}}^{\dagger} \\
\tilde{p}_{\vec{n}}^{\dagger} \\
\tilde{p}_{\vec{n}}^{\dagger}
\end{array}\right], \quad \xi_{\vec{n}}^{\mathbb{R}} \equiv\left[\begin{array}{c}
q_{\vec{n}} \\
\bar{q}_{\vec{n}} \\
p_{\vec{n}} \\
\bar{p}_{\vec{n}}
\end{array}\right], \\
R_{\mathbb{C} \rightarrow \mathbb{R}} \equiv \frac{1}{2}\left[\begin{array}{cccc}
1 & 1 & i \lambda_{n}^{-1} & -i \lambda_{n}^{-1} \\
1 & 1 & -i \lambda_{n}^{-1} & i \lambda_{n}^{-1} \\
-i \lambda_{n} & i \lambda_{n} & 1 & 1 \\
i \lambda_{n} & -i \lambda_{n} & 1 & 1
\end{array}\right] .
\end{aligned}
$$

This transformation of phase space operators does not modify the commutation relations (2.5) since it satisfies the condition (2.31). It is also easy to check explicitly that this transformation generates real operators $\xi_{n}^{\mathbb{R}}=\left(\xi_{n}^{\mathbb{R}}\right)^{\dagger} \cdot{ }^{14}$ By substituting this coordinate transformation into eq. (3.20), we obtain

$$
\begin{aligned}
H & =\frac{1}{2} \sum_{\vec{n}} \omega_{n}\left[\lambda_{n}^{-1}\left(p_{\vec{n}}^{2}+\bar{p}_{\vec{n}}^{2}\right)+\lambda_{n}\left(q_{\vec{n}}^{2}+\bar{q}_{\vec{n}}^{2}\right)\right], \\
C & =\frac{1}{2} \sum_{\vec{n}}\left[\lambda_{n}^{-1}\left(p_{\vec{n}}^{2}-\bar{p}_{\vec{n}}^{2}\right)+\lambda_{n}\left(q_{\vec{n}}^{2}-\bar{q}_{\vec{n}}^{2}\right)\right] .
\end{aligned}
$$

Here, we see that the oscillators remain decoupled in the expressions for the Hamiltonian and the charge, where the oscillators $\left(q_{\vec{n}}, p_{\vec{n}}\right)$ have positive charge while $\left(\bar{q}_{\vec{n}}, \bar{p}_{\vec{n}}\right)$ have negative charge, cf. eq. (3.16).

In order to gain physical intuition for this decomposition, it is instructive to consider the above transformations in terms of creation and annihilation operators. Written in terms of creation and annihilation operators, the phase space operators introduced previously read

$$
\begin{aligned}
q_{\vec{n}} & =\sqrt{\frac{1}{2 \lambda_{n}}}\left(a_{\vec{n}}+a_{\vec{n}}^{\dagger}\right), & p_{\vec{n}} & =-i \sqrt{\frac{\lambda_{n}}{2}}\left(a_{\vec{n}}-a_{\vec{n}}^{\dagger}\right), \\
\bar{q}_{-\vec{n}} & =\sqrt{\frac{1}{2 \lambda_{n}}}\left(\bar{a}_{\vec{n}}+\bar{a}_{\vec{n}}^{\dagger}\right), & \bar{p}_{-\vec{n}} & =-i \sqrt{\frac{\lambda_{n}}{2}}\left(\bar{a}_{\vec{n}}-\bar{a}_{\vec{n}}^{\dagger}\right) .
\end{aligned}
$$

Thus, $\left(q_{\vec{n}}, p_{\vec{n}}\right)$ and $\left(\bar{q}_{\vec{n}}, \bar{p}_{\vec{n}}\right)$ correspond to the real phase space operators for particles and anti-particles of the field theory, respectively. This explains the signs of the charge contributions in (3.23) from $\left(q_{\vec{n}}, p_{\vec{n}}\right)$ and $\left(\bar{q}_{\vec{n}}, \bar{p}_{\vec{n}}\right)$. Furthermore, the transformation in eq. (3.22)

\footnotetext{
${ }^{14}$ Here, when we write $\xi^{\dagger}$, we mean that we simply Hermitian conjugate the contents of $\xi$ without additionally transposing $\xi$ between being a row vector and a column vector.
} 
can be decomposed into a rotation of the complex phase space operators to real phase space operators as in (2.18), followed by a symplectic transformation which separates the particles' and anti-particles' creation and annihilation operators. The set of operators $\xi^{\mathbb{R}}$ in eq. (3.22) will allow us to directly utilize certain covariance matrices from [11] which we review in section 4.1 below, while the use of $\xi^{\mathbb{C}}$ will allow us to more naturally evaluate the covariance matrix for the reference state, see eq. (4.27) below. Finally, the transformations between these two bases will allow us to freely move between the different bases as we evaluate the complexity.

Before moving on, let us take a moment to compare the transformations introduced in this section and those introduced for the uncharged TFD problem for a real scalar field [11]. Due to the lack of charge and the reality of their field, the authors of [11] were content to stop at (3.20) after performing the Fourier transform - note that, modulo Hermitian conjugation, $H$ is decoupled in the tilde phase space operators there. ${ }^{15}$ It is the second part of the symplectic transformation (3.22), which splits the particles and anti-particles degrees of freedom, which was not needed for the case of the uncharged TFD. We will find this transformation crucial later to the decomposition of charged oscillator TFDs to uncharged TFDs.

\subsection{The reference state}

The complexity problem involves a reference state, see discussion around eq. (2.21). The reference state serves as the starting point for our quantum circuits and is usually chosen to be simple in the sense that it is unentangled. It was proposed in $[6,7]$ that a natural reference state for complexity in QFTs is the ground state of some Hamiltonian

$$
H_{\mathrm{ref}} \equiv \int d^{d-1} x\left(\pi^{\dagger} \pi+m_{\mathrm{ref}}^{2} \phi^{\dagger} \phi\right)
$$

given in terms of a different frequency $m_{\text {ref }}$, known as the reference state scale. ${ }^{16}$ Note that, due to the lack of the derivative term compared to eq. (3.1), this Hamiltonian only couples the various position and momentum degrees of freedom - $\phi(x), \pi(x)-$ at the same spatial point $x$ rather than introducing a more complicated structure where the degrees of freedom at different points are coupled to each other. Hence, the vacuum state for the Hamiltonian (3.26) is spatially unentangled - a desirable property for a 'simple' reference state.

Note that, in the absence of a UV regulator, e.g., a lattice, the energy of the reference state of the QFT diverges in the UV (with respect to the physical Hamiltonian) and therefore resides outside the 'physical' Hilbert space of states whose energy is UV-finite. The motivation behind this definition of the reference state in $[6,7]$ stems from the fact that physical states in QFT have correlations down to arbitrary short distance scales, and therefore it is expected that those states are infinitely more complex than the unentangled

\footnotetext{
${ }^{15}$ Actually, the Hamiltonian of [11] had a very similar form to (3.20), but with $n$ and $-n$ modes mixed. This is because for the case of a real field we have $q_{\vec{n}}^{\dagger}=q_{-\vec{n}}$ and $p_{\vec{n}}^{\dagger}=p_{-\vec{n}}$. These can be decoupled as in appendix D of [11] by performing a coordinate transformation of the type (2.18).

${ }^{16} m_{\text {ref }}$ was denoted $\mu$ in [11], which we have changed in order to reserve $\mu$ for the chemical potential.
} 
reference state. It is therefore sensible to have a reference state which is "infinitely far" from our target states. A finite notion of complexity can only be defined in the presence of a UV-regulator (e.g., on the lattice). This is similar to what happens for the entanglement entropy which is also a UV-divergent quantity in the continuum QFT. Once a lattice regulator is introduced, our reference and target states both live in the same Hilbert space. As discussed in the introduction, UV-divergences in the complexity can be regarded as a constant overhead which cancels when comparing the complexity of different states in the physical Hilbert space (e.g., when evaluating the complexity of formation).

The Hamiltonian in eq. (3.26) can be discretized and expressed in terms of the operators (3.18) yielding

$$
H_{\text {ref }}=\sum_{\vec{n}} m_{\text {ref }}\left[\lambda_{\text {ref }}^{-1} \tilde{p}_{\vec{n}}^{\dagger} \tilde{p}_{\vec{n}}+\lambda_{\text {ref }} \tilde{q}_{\vec{n}}^{\dagger} \tilde{q}_{\vec{n}}\right]
$$

where above we have defined

$$
\lambda_{\text {ref }} \equiv \frac{m_{\text {ref }}}{\mu_{g}} .
$$

Recall from the discussion around eq. (2.27) that the straight line trajectory is optimal with respect to the $F_{2}$ norm and provides a good approximation for the optimal trajectory with respect to the $F_{1}$ norm when the reference state scale and the gate scale are equal, i.e., when $\lambda_{\text {ref }}=1$ [11]. Applying the extra coordinate transformation (3.22) decoupling the particles and anti-particles unfortunately does not preserve the simple form of the reference Hamiltonian but rather yields:

$$
\begin{aligned}
H_{\text {ref }}= & \sum_{\vec{n}} m_{\text {ref }}\left[\lambda_{\text {ref }}^{-1} \tilde{p}_{\vec{n}}^{\dagger} \tilde{p}_{s, \vec{n}}+\lambda_{\text {ref }} \tilde{q}_{\vec{n}}^{\dagger} \tilde{q}_{\vec{n}}\right] \\
= & \frac{1}{4} \sum_{\vec{n}} m_{\text {ref }}\left\{\lambda_{\text {ref }}^{-1}\left(p_{\vec{n}}+\bar{p}_{\vec{n}}\right)^{2}+\frac{\lambda_{\text {ref }}}{\lambda_{n}^{2}}\left(p_{\vec{n}}-\bar{p}_{\vec{n}}\right)^{2}\right. \\
& \left.+\lambda_{\text {ref }}\left(q_{\vec{n}}+\bar{q}_{\vec{n}}\right)^{2}+\frac{\lambda_{n}^{2}}{\lambda_{\text {ref }}}\left(q_{\vec{n}}-\bar{q}_{\vec{n}}\right)^{2}\right\} .
\end{aligned}
$$

We will therefore be mostly using the complex coordinates $\xi^{\mathbb{C}}$ when discussing the reference state.

\section{Complexity of the cTFD state}

Having separately discussed complexity and the complex scalar field theory in sections 2 and 3, respectively, in this section, we combine the machinery developed thus far, in order to compute the complexity of the cTFD state of two copies of a complex scalar field theory. We begin in subsection 4.1 with a review of the properties of the uncharged TFD state of two harmonic oscillators [11], specifically noting the expression for its covariance matrix. In subsection 4.2, we next consider the cTFD state of two complex harmonic oscillators, finding that this state factorizes to two uncharged TFDs. The upshot is that we 
may reuse the covariance matrix of the uncharged TFD for the cTFD, by merely shifting the temperatures and times according to the chemical potential — we explain this in subsection 4.3. ${ }^{17}$ In subsection 4.4, we use the decomposition of the complex scalar field into harmonic oscillators, as discussed in subsections 3.2-3.3, to argue that the complexity of the complex scalar cTFD is simply the harmonic oscillator answer summed over the modes of the scalar. Finally, in subsections 4.5 and 4.6, we use these results to compute the complexity of formation and the time evolution of complexity for the complex scalar, respectively.

\subsection{Properties of the uncharged thermofield double state}

Before we consider the cTFD state, let us briefly summarize some useful results from [11] about the uncharged TFD state of two harmonic oscillators. The state is a purification of a mixed thermal state on a system with two copies, just like eq. (1.1), but without chemical potential and charge. More explicitly, it is defined as

$$
\left|\operatorname{TFD}\left(t_{L}, t_{R}\right)\right\rangle=\frac{1}{\sqrt{Z_{\beta}}} \sum_{n} e^{-\beta E_{n} / 2} e^{-i E_{n}\left(t_{L}+t_{R}\right)}\left|E_{n}\right\rangle_{L}\left|E_{n}\right\rangle_{R}
$$

where $t_{L}$ and $t_{R}$ are the times on the two identical copies, $\beta$ is the inverse temperature and $\left|E_{n}\right\rangle_{L}$ and $\left|E_{n}\right\rangle_{R}$ are energy eigenstates of the left and right harmonic oscillators respectively, with energy eigenvalues $E_{n} .{ }^{18}$ We will consider a single mode/oscillator in the left system with (dimensionless) position and momentum denoted $\left(q_{L}, p_{L}\right)$ and a single harmonic oscillator in the right system with position and momentum denoted $\left(q_{R}, p_{R}\right)$, both taken to have the same frequency $\omega$. The Hamiltonian for this system is given by

$$
H=\sum_{s \in[L, R]} \frac{\omega}{2}\left(\lambda^{-1} p_{s}^{2}+\lambda q_{s}^{2}\right)=\sum_{s \in[L, R]} \omega\left(a_{s}^{\dagger} a_{s}+\frac{1}{2}\right)
$$

where $\lambda$ is a parameter encoding the mass of the oscillators and where here the phase space operators are real and are related to the creation and annihilation operators according to

$$
q_{s}=\frac{1}{\sqrt{2 \lambda}}\left(a_{s}^{\dagger}+a_{s}\right), \quad p_{s}=i \sqrt{\frac{\lambda}{2}}\left(a_{s}^{\dagger}-a_{s}\right), \quad s \in[L, R]
$$

From now on, we will use a subscript $s \in[L, R]$ on our variables, indicating which copy we are referring to. Note that eq. (4.1) depends on the combination of times $t_{L}+t_{R}$ and so the full time dependence can be captured by setting, e.g., $t_{L}=t_{R}=t / 2$ as we do in the following. The time dependent TFD state of the two harmonic oscillators is given by the

\footnotetext{
${ }^{17}$ Note however that while we are able to adapt the TFD covariance matrices from [6] to the case of the cTFD by using the particle anti-particle factorization, the result for the complexity cannot be adapted directly from the uncharged case due to the non-trivial structure of the reference state of the charged system in the particle anti-particle basis, cf. eq. (3.29).

${ }^{18}$ Here again, we follow the common nomenclature of referring to the two harmonic oscillators as the "left" and "right" copies.
} 
following explicit expression

$$
\begin{aligned}
|\operatorname{TFD}(t)\rangle & =Z_{\beta}^{-1 / 2} \sum_{n=0}^{\infty} e^{-\frac{\beta \omega}{2}\left(n+\frac{1}{2}\right)} e^{-i \omega\left(n+\frac{1}{2}\right) t}|n\rangle_{L}|n\rangle_{R} \\
& =Z_{\beta}^{-1 / 2} e^{-\frac{\beta \omega}{4}} e^{-\frac{i}{2} \omega t} \sum_{n=0}^{\infty} \exp \left[e^{-\beta \omega / 2} e^{-i \omega t} a_{L}^{\dagger} a_{R}^{\dagger}\right]|0\rangle_{L}|0\rangle_{R}
\end{aligned}
$$

where the normalization factor is defined as $Z_{\beta} \equiv e^{-\beta \omega / 2}\left(1-e^{-\beta \omega}\right)^{-1}$.

The TFD state takes a simpler form in the "diagonal" \pm basis mentioned below eq. (2.27), mixing the two sides, defined according to

$$
q_{ \pm} \equiv \frac{1}{\sqrt{2}}\left(q_{L} \pm q_{R}\right), \quad p_{ \pm} \equiv \frac{1}{\sqrt{2}}\left(p_{L} \pm p_{R}\right),
$$

i.e.,

$$
\left[\begin{array}{l}
q_{+} \\
q_{-}
\end{array}\right]=R_{L R \rightarrow \pm}\left[\begin{array}{l}
q_{L} \\
q_{R}
\end{array}\right], \quad\left[\begin{array}{l}
p_{+} \\
p_{-}
\end{array}\right]=R_{L R \rightarrow \pm}\left[\begin{array}{l}
p_{L} \\
p_{R}
\end{array}\right], \quad R_{L R \rightarrow \pm} \equiv \frac{1}{\sqrt{2}}\left[\begin{array}{cc}
1 & 1 \\
1 & -1
\end{array}\right] .
$$

In this basis, the TFD state can be written as follows (see eqs. (35), (36) and (77) in [11])

$$
\begin{aligned}
|\operatorname{TFD}(t)\rangle & =e^{-i \alpha \hat{\mathcal{O}}_{+}(t)}|0\rangle_{+} \otimes e^{i \alpha \hat{\mathcal{O}}_{-}(t)}|0\rangle_{-} \\
\hat{\mathcal{O}}_{ \pm}(t) & \equiv \frac{1}{2} \cos (\omega t)\left(q_{ \pm} p_{ \pm}+p_{ \pm} q_{ \pm}\right)+\frac{1}{2} \sin (\omega t)\left(\lambda q_{ \pm}^{2}-\lambda^{-1} p_{ \pm}^{2}\right)
\end{aligned}
$$

where we have defined

$$
\alpha \equiv \frac{1}{2} \log \left(\frac{1+e^{-\beta \omega / 2}}{1-e^{-\beta \omega / 2}}\right) .
$$

The covariance matrix of the TFD state in the \pm basis is given by eq. (76) of [11], i.e.,

$$
G_{\mathrm{TFD}}^{ \pm}(t, \alpha) \equiv\left[\begin{array}{cc}
\lambda^{-1}[\cosh (2 \alpha) \pm \sinh (2 \alpha) \cos (\omega t)] & \mp \sinh (2 \alpha) \sin (\omega t) \\
\mp \sinh (2 \alpha) \sin (\omega t) & \lambda[\cosh (2 \alpha) \mp \sinh (2 \alpha) \cos (\omega t)]
\end{array}\right] .
$$

This TFD state consists of a single Harmonic oscillator of frequency $\omega$.

When studying the uncharged TFD state of a real scalar QFT, [11] have shown that the problem factorizes to evaluating the complexity of a product of different one-mode TFD states, each with a different frequency $\omega_{k}=\sqrt{k^{2}+m^{2}}$ where $k$ is the spatial momentum of the different modes and $m$ is the QFT mass.

\section{2 cTFD of two complex harmonic oscillators}

Next, we consider the charged thermofield double consisting of two complex or four real harmonic oscillators. We will label each oscillator as right or left $(R$ or $L)$ and particle or anti-particle (no overbar or overbar). The complete Hamiltonian is given by

$$
H=H_{R}+H_{L} ; \quad H_{s}=\frac{\omega}{2}\left[\lambda^{-1}\left(p_{s}^{2}+\bar{p}_{s}^{2}\right)+\lambda\left(q_{s}^{2}+\bar{q}_{s}^{2}\right)\right], \quad s \in[L, R] .
$$


This is to be representative of a single mode in eq. (3.23). Alternatively, in terms of complex phase space operators we have $H_{s}=\omega\left(\lambda^{-1} \tilde{p}_{s}^{\dagger} \tilde{p}_{s}+\lambda \tilde{q}_{s}^{\dagger} \tilde{q}_{s}\right)$. We may use similar creation and annihilation operators to those in eq. (3.24) to expand the Hamiltonian and charge, i.e., we take ${ }^{19}$

$$
\begin{aligned}
q_{s} & =\sqrt{\frac{1}{2 \lambda}}\left(a_{s}+a_{s}^{\dagger}\right), & p_{s} & =-i \sqrt{\frac{\lambda}{2}}\left(a_{s}-a_{s}^{\dagger}\right), \\
\bar{q}_{s} & =\sqrt{\frac{1}{2 \lambda}}\left(\bar{a}_{s}+\bar{a}_{s}^{\dagger}\right), & \bar{p}_{s} & =-i \sqrt{\frac{\lambda}{2}}\left(\bar{a}_{s}-\bar{a}_{s}^{\dagger}\right),
\end{aligned}
$$

and define the number operators as in (3.17)

$$
N_{s} \equiv a_{s}^{\dagger} a_{s}, \quad \bar{N}_{s} \equiv \bar{a}_{s}^{\dagger} \bar{a}_{s}
$$

In terms of creation $a_{s}^{\dagger}, \bar{a}_{s}^{\dagger}$, annihilation $a_{s}, \bar{a}_{s}$, and number $N_{s}, \bar{N}_{s}$, operators, we have

$$
H_{s}=\omega\left(N_{s}+\bar{N}_{s}+1\right) \text {. }
$$

Similarly, the total charge of a given side is given by

$$
C_{s}=\frac{1}{2}\left[\lambda^{-1}\left(q_{s}^{2}-\bar{q}_{s}^{2}\right)+\lambda\left(p_{s}^{2}-\bar{p}_{s}^{2}\right)\right]=N_{s}-\bar{N}_{s} .
$$

Alternatively, in terms of complex phase space operators we have $C_{s}=i\left(\tilde{q}_{s}^{\dagger} \tilde{p}_{s}^{\dagger}-\tilde{q}_{s} \tilde{p}_{s}\right)$. We denote eigenstates of the number operators by $|n, \bar{n}\rangle_{s}$, satisfying

$$
N_{s}|n, \bar{n}\rangle_{s}=n|n, \bar{n}\rangle_{s}, \quad \bar{N}_{s}|n, \bar{n}\rangle_{s}=\bar{n}|n, \bar{n}\rangle_{s}
$$

The creation and annihilation operators raise and lower number eigenvalues according to

$$
|n, \bar{n}\rangle_{s}=\frac{\left(a_{s}^{\dagger}\right)^{n}\left(\bar{a}_{s}^{\dagger}\right)^{\bar{n}}}{\sqrt{n ! \bar{n} !}}|0,0\rangle_{s}
$$

which have energy and charge eigenvalues given by

$$
\begin{aligned}
H_{s}|n, \bar{n}\rangle_{s} & =E_{n, \bar{n}}|n, \bar{n}\rangle_{s}, & E_{n, \bar{n}} & =\omega(n+\bar{n}+1), \\
C_{s}|n, \bar{n}\rangle_{s} & =c_{n, \bar{n}}|n, \bar{n}\rangle_{s}, & c_{n, \bar{n}} & =n-\bar{n} .
\end{aligned}
$$

The cTFD state, is defined in general by eq. (1.1). As in the uncharged case of subsection 4.1 , let us again set $t_{L}=t_{R}=t / 2$. Then, specializing eq. (1.1) to the present theory of harmonic oscillators, with energy and charge eigenstates and eigenvalues given in eq. (4.18), we find, in analogy to eq. (4.4),

$$
\begin{aligned}
& |\operatorname{cTFD}(\beta, \mu ; t, \omega)\rangle=Z_{\beta, \mu}^{-1 / 2} \sum_{n, \bar{n}=0}^{\infty} \exp \left\{-\left(\frac{\beta}{2}+i t\right)\left(E_{n, \bar{n}}+\mu c_{n, \bar{n}}\right)\right\}|n, \bar{n}\rangle_{L}|\bar{n}, n\rangle_{R} \\
& \quad=Z_{\beta, \mu}^{-1 / 2} e^{-\omega\left(\frac{\beta}{2}+i t\right)} \sum_{n, \bar{n}=0}^{\infty} \exp \left\{-\left(\frac{\beta}{2}+i t\right)[\omega(n+\bar{n})+\mu(n-\bar{n})]\right\}|n, \bar{n}\rangle_{L}|\bar{n}, n\rangle_{R} .
\end{aligned}
$$

\footnotetext{
${ }^{19}$ Here $a_{s}$ is identified with $a_{s, \vec{n}}$ while $\bar{a}_{s}$ is identified with $\bar{a}_{s,-\vec{n}}$ from the previous discussion around eq. (3.24).
} 
Note the ordering of $\bar{n}, n$ in writing $|\bar{n}, n\rangle_{R}$; by this, we mean the $\bar{n}$-th eigenstate of $N_{R}$ and the $n$-th eigenstate of $\bar{N}_{R}$. This was done in order to recover the structure in eq. (1.1) with opposite charges on the left and right sides. The normalization constant $Z_{\beta, \mu}$ is given by

$$
\begin{aligned}
Z_{\beta, \mu} & =e^{-\beta \omega} \sum_{n, \bar{n}=0}^{\infty} \exp \{-\beta[\omega(n+\bar{n})+\mu(n-\bar{n})]\} \\
& =e^{-\beta \omega}\left[1-e^{-\beta(\omega-\mu)}\right]^{-1}\left[1-e^{-\beta(\omega+\mu)}\right]^{-1} \\
& =e^{-\beta \omega}\left[1+e^{-2 \beta \omega}-2 e^{-\beta \omega} \cosh (\beta \mu)\right]^{-1}
\end{aligned}
$$

Comparison of (4.19) with (4.4) shows that this cTFD is just a product of two uncharged TFD states

$$
|\operatorname{cTFD}(\beta, \mu ; t, \omega)\rangle=\left|\operatorname{TFD}\left(\beta_{\bar{L} R}, \mu ; t_{\bar{L} R}\right)\right\rangle_{\bar{L} R} \otimes\left|\operatorname{TFD}\left(\beta_{L \bar{R}}, \mu ; t_{L \bar{R}}\right)\right\rangle_{L \bar{R}}
$$

at temperatures and times shifted by the chemical potential ${ }^{20}$

$$
\begin{aligned}
\beta_{\bar{L} R} \equiv \beta\left(1-\frac{\mu}{\omega}\right), & t_{\bar{L} R} \equiv t\left(1-\frac{\mu}{\omega}\right), \\
\beta_{L \bar{R}} \equiv \beta\left(1+\frac{\mu}{\omega}\right), & t_{L \bar{R}} \equiv t\left(1+\frac{\mu}{\omega}\right) .
\end{aligned}
$$

Thus, we see that we can use the covariance matrices of the uncharged TFD from reference [11], to study the complexity of the cTFD.

From eqs. (4.22) and (4.23), we already see something interesting. As $|\mu| \rightarrow \omega$, one of the effective temperatures $\beta_{\bar{L} R}^{-1}, \beta_{L \bar{R}}^{-1}$ blows up; further for $|\mu|>\omega$, we have an ill-defined negative-effective-temperature state in either $\bar{L} R$ or $L \bar{R}$. In particular, notice that, for $|\mu|>$ $\omega$, the cTFD state (4.19) becomes ill-defined since the normalization factor (4.20) diverges. This will force us to take all frequencies in our field theory construction, see eq. (3.12), to be at least as large as the chemical potential and, in particular, this implies that in our field theory setup we must have $m \geq|\mu| .{ }^{21}$ This means that we will not be able to reach the conformal limit for fixed $\mu \neq 0$ in this work. Moreover, note that in general replacing $\mu \leftrightarrow-\mu$ is equivalent to swapping $\bar{L} R \leftrightarrow L \bar{R}$, under which the complexity is invariant.

\subsection{Complexity of the cTFD of two complex harmonic oscillators}

We now have the necessary ingredients to compute the complexity of the charged thermofield double state (4.19) of the two complex harmonic oscillator system. Recall from the paragraph above eq. (2.28) that the complexity depends on the choice of basis, and

\footnotetext{
${ }^{20}$ Note that this cannot be rephrased purely as a shift in the frequencies $\omega_{L \bar{R}} \equiv \omega+\mu, \omega_{\bar{L} R} \equiv \omega-\mu$ since the states $|n, \bar{n}\rangle_{s}$ used to construct the cTFD in eq. (4.19) are created from the vacuum state of each side $|0, \overline{0}\rangle$ with the creation operators defined with respect to the Hamiltonian containing the original frequency of the theory. Therefore, in (4.9), it is the case that, while $\alpha$ becomes shifted due to the modified temperatures in eq. (4.22)-(4.23), the parameter $\lambda$ is defined with the frequency of the original theory rather than with the shifted frequencies.

${ }^{21}$ The case $m=|\mu|$ is rather singular, however as we explained in footnote 8 and revisit below in footnote 27 , this limit can be taken smoothly in the field theory in sufficiently high dimensions.
} 
that it was found in [11] that the $F_{1}$ cost function with a choice of basis which does not mix the left and right degrees of freedom was the one which reproduced best a number of qualitative features of complexity in holography. We have chosen to focus on a similar choice of bases below. We will consider two different bases, the complex left-right basis $L R_{\mathbb{C}}$ corresponding to operators

$$
\xi^{L R_{\mathbb{C}}} \equiv\left[\tilde{q}_{L}, \tilde{q}_{L}^{\dagger}, \tilde{q}_{R}, \tilde{q}_{R}^{\dagger}, \tilde{p}_{L}^{\dagger}, \tilde{p}_{L}, \tilde{p}_{R}^{\dagger}, \tilde{p}_{R}\right],
$$

and the real left-right basis $L R$ corresponding to the particle and anti-particle degrees of freedom

$$
\xi^{L R} \equiv\left[q_{L}, \bar{q}_{L}, q_{R}, \bar{q}_{R}, p_{L}, \bar{p}_{L}, p_{R}, \bar{p}_{R}\right],
$$

where $\xi^{L R_{\mathbb{C}}}$ is related to $\xi^{L R}$ by eq. (3.22). ${ }^{22}$ (To reuse the results of subsection 4.1, we will also need to relate the above to a "diagonal" \pm basis (cf. eq. (4.5)), as we do below; however, we will always transform back to the $\xi^{L R_{\mathbb{C}}}$ or $\xi^{L R}$ bases to compute complexity.)

For the reference state, we take the vacuum of a single mode of the complex scalar reference Hamiltonian (3.27):

$$
H_{R, \text { ref }}=m_{\text {ref }}\left(\lambda_{\text {ref }}^{-1} \tilde{p}_{R}^{\dagger} \tilde{p}_{R}+\lambda_{\text {ref }} \tilde{q}_{R}^{\dagger} \tilde{q}_{R}\right) .
$$

The covariance matrix of the reference state in the $L R_{\mathbb{C}}$ basis can be evaluated directly from the definition (2.7) by using creation and annihilation operators adapted to the reference state as in eq. (3.18) with the replacement $\lambda_{n} \rightarrow \lambda_{\text {ref }}$ which yields

$$
G_{\text {ref }}^{L_{\mathbb{C}}}=G_{\text {ref }}^{R_{\mathbb{C}}}=\operatorname{diag}\left(\lambda_{\text {ref }}^{-1}, \lambda_{\text {ref }}^{-1}, \lambda_{\text {ref }}, \lambda_{\text {ref }}\right),
$$

where we have used the superscripts $L_{\mathbb{C}}$ and $R_{\mathbb{C}}$ to denote the complex operators for the left and right copies (according to the order in eq. (3.22)), respectively. The full covariance matrix for the reference state will simply be the direct sum of those two 4 by 4 matrices according to the order of operators in eq. (4.24).

Using the decomposition (4.21) of the cTFD into uncharged TFDs, we can deduce the (target) covariance matrices of the cTFD. It is helpful here to define the symbol $\tilde{\oplus}$ to mean direct sum, followed by reordering rows and columns so that positions are listed before momenta. With this, the covariance matrix of the cTFD is given by

$$
G_{\mathrm{TFD}}^{+}\left(t_{\bar{L} R, n}, \alpha_{\bar{L} R, n}\right) \tilde{\oplus} G_{\mathrm{TFD}}^{+}\left(t_{L \bar{R}, n}, \alpha_{L \bar{R}, n}\right) \tilde{\oplus} G_{\mathrm{TFD}}^{-}\left(t_{\bar{L} R, n}, \alpha_{\bar{L} R, n}\right) \tilde{\oplus} G_{\mathrm{TFD}}^{-}\left(t_{L \bar{R}, n}, \alpha_{L \bar{R}, n}\right)
$$

where $G_{\mathrm{TFD}}^{ \pm}$is the uncharged TFD covariance matrix (4.9), with the shifted times $t_{\bar{L} R, n}, t_{L \bar{R}, n}$, and $\alpha_{\bar{L} R}, \alpha_{L \bar{R}}$ from eq. (4.8) defined in terms of the shifted temperatures $\beta_{\bar{L} R}, \beta_{L \bar{R}}$ given in eqs. (4.22)-(4.23). The covariance matrix obtained in this way will be given in the following basis of operators

$$
\xi^{ \pm} \equiv\left[q_{\bar{L} R}^{+}, q_{L \bar{R}}^{+}, q_{\bar{L} R}^{-}, q_{L \bar{R}}^{-}, p_{\bar{L} R}^{+}, p_{L \bar{R}}^{+}, p_{\bar{L} R}^{-}, p_{L \bar{R}}^{-}\right],
$$

\footnotetext{
${ }^{22}$ Of course, here we mean that the operators are related by a matrix constructed as a direct sum of (3.22) for the left and right copies.
} 
obtained from eq. (4.25) by the equivalent change of coordinates to the one in eqs. (4.5)(4.6), which mixes the $\bar{L} R$ operators and the $\bar{R} L$ operators separately, i.e.,

$$
\begin{array}{ll}
q_{\bar{L} R}^{ \pm} \equiv \frac{1}{\sqrt{2}}\left(\bar{q}_{L} \pm q_{R}\right), & p_{\bar{L} R}^{ \pm} \equiv \frac{1}{\sqrt{2}}\left(\bar{p}_{L} \pm p_{R}\right), \\
q_{L \bar{R}}^{ \pm} \equiv \frac{1}{\sqrt{2}}\left(q_{L} \pm \bar{q}_{R}\right), & p_{L \bar{R}}^{ \pm} \equiv \frac{1}{\sqrt{2}}\left(p_{L} \pm \bar{p}_{R}\right) .
\end{array}
$$

The relevant circuits will consist of $8 \times 8$ matrices acting on covariance matrices according to $(2.20)-(2.21)$ where $S(\sigma) \in \operatorname{Sp}(8, \mathbb{R})$. In particular, we shall consider the straight line circuit described around eqs. (2.26)-(2.27). We can compute the relative covariance matrix (2.26) for the cTFD state in, say the $L R$ basis, as follows

$$
\begin{aligned}
& \Delta_{\mathrm{CTFD}}^{L R}= \\
& R_{ \pm \rightarrow L R}\left[G_{\mathrm{TFD}}^{+}\left(t_{\bar{L} R}, \alpha_{\bar{L} R}\right) \tilde{\oplus} G_{\mathrm{TFD}}^{+}\left(t_{L \bar{R}}, \alpha_{L \bar{R}}\right) \tilde{\oplus} G_{\mathrm{TFD}}^{-}\left(t_{\bar{L} R}, \alpha_{\bar{L} R}\right) \tilde{\oplus} G_{\mathrm{TFD}}^{-}\left(t_{L \bar{R}}, \alpha_{L \bar{R}}\right)\right] R_{ \pm \rightarrow L R}^{\dagger} \\
& \quad \cdot\left[\left(R_{L_{\mathbb{C}} \rightarrow L} G_{\mathrm{ref}}^{L_{\mathbb{C}}} R_{L_{\mathbb{C}} \rightarrow L}^{\dagger}\right) \tilde{\oplus}\left(R_{R_{\mathbb{C}} \rightarrow R} G_{\mathrm{ref}}^{R_{\mathbb{C}}} R_{R_{\mathbb{C}} \rightarrow R}^{\dagger}\right)\right]^{-1}
\end{aligned}
$$

where

$$
\xi^{L R}=R_{ \pm \rightarrow L R} \xi^{ \pm}, \quad R_{ \pm \rightarrow L R}=R_{4} \oplus R_{4}, \quad R_{4}=\frac{1}{\sqrt{2}}\left[\begin{array}{cccc}
0 & 1 & 0 & 1 \\
1 & 0 & 1 & 0 \\
1 & 0 & -1 & 0 \\
0 & 1 & 0 & -1
\end{array}\right] \in \mathrm{SO}(4)
$$

inverts the transformation (4.30) and $R_{L_{\mathbb{C}} \rightarrow L}=R_{R_{\mathbb{C}} \rightarrow R}$ is given in eq. (3.22) applied to the left and right operators, respectively. We can easily transform $\Delta_{\text {cTFD }}^{L R}$ to another basis, say the $L R_{\mathbb{C}}$ basis as follows

$$
\Delta_{\mathrm{cTFD}}^{L R_{\mathbb{C}}}=\left(R_{L \rightarrow L_{\mathbb{C}}} \tilde{\oplus} R_{R \rightarrow R_{\mathbb{C}}}\right) \Delta_{\mathrm{cTFD}}^{L R}\left(R_{L \rightarrow L_{\mathbb{C}}} \tilde{\oplus} R_{R \rightarrow R_{\mathbb{C}}}\right)^{-1},
$$

where $R_{L \rightarrow L_{\mathbb{C}}}=R_{R \rightarrow R_{\mathbb{C}}}$ is given by inverting eq. (3.22). It only remains to compute (the upper bound for) the $F_{1}$ complexity via eq. (2.29) with an appropriate basis $K_{I}$ for $\mathfrak{s p}(8)$, see appendix A. We reiterate this equation here for convenience

$$
\mathcal{C}_{1}^{\mathrm{UB}}=\frac{1}{4} \sum_{I}\left|\operatorname{tr}\left(\log \left(\Delta_{\mathrm{cTFD}}\right) \cdot K_{I}^{\dagger}\right)\right|,
$$

where UB stands for "upper bound".

We have not written the explicit expression here, since this expression arising from the product of the $8 \times 8$ matrices above is cumbersome and not particularly illuminating, but it is easily obtained by substituting into eq. (4.34) the generators in the complex basis given in appendix A and the relative covariance matrix (4.33) given explicitly in terms of eqs. (3.22), (4.8)-(4.9), (4.22)-(4.23), (4.27) and (4.31)-(4.32). We will study various properties of this (upper bound on the) complexity analytically in certain limits as well as numerically. 


\section{4 cTFD states of the complex scalar}

The cTFD state for the complex scalar theory is defined according to eq. (1.1). As in section 4.1 we will set $t_{L}=t_{R}=t / 2$ (but the full time dependence can easily be recovered, given that the time dependence is simply dictated by the sum of the two times). The relevant state for our construction is therefore given by

$$
|\operatorname{cTFD}(\beta, \mu ; t)\rangle \equiv \frac{1}{\sqrt{Z_{\beta, \mu}}} \sum_{n, \sigma} e^{-\frac{\beta}{2}\left(E_{n}+\mu c_{\sigma}\right)-i\left(E_{n}+\mu c_{\sigma}\right) t}\left|E_{n}, c_{\sigma}\right\rangle_{L}\left|E_{n},-c_{\sigma}\right\rangle_{R},
$$

with $\left|E_{n}, c_{\sigma}\right\rangle$ denoting the Hamiltonian and charge eigenstates with eigenvalues $E_{n}, c_{\sigma}$, respectively. The time evolution in eq. (4.35) is motivated in appendix B. The goal of this section is to calculate the complexity of this state for free complex scalar field theory.

The decomposition (3.12) together with the commutation relations (3.11) teach us that the cTFD state in the complex scalar field theory factorizes into cTFD states of each of the momentum modes, i.e.,

$$
|\operatorname{cTFD}(\beta, \mu ; t)\rangle=\bigotimes_{\vec{n}}\left|\operatorname{cTFD}_{n}\left(\beta, \mu ; t, \omega_{n}\right)\right\rangle,
$$

where the frequencies $\omega_{n}$ were defined in eq. (3.12). Due to the mode factorization (4.36) of the complex scalar cTFD, the circuit constructing it will similarly factor into circuits which act separately on each mode $\vec{n}$. As described in sections 3.2-3.3, each of the momentum modes of the complex scalar is equivalent to a complex harmonic oscillator. Therefore, we may compute the complexity of the complex scalar cTFD by summing the complexities of harmonic oscillator cTFDs - the calculation of which was described in section $4.3-$ according to

$$
\mathcal{C}(|\operatorname{cTFD}(\beta, \mu ; t)\rangle)=\sum_{\vec{n}} \mathcal{C}\left(\left|\operatorname{cTFD}_{n}\left(\beta, \mu ; t, \omega_{n}\right)\right\rangle\right) .
$$

Eq. (4.37), with the sum over discrete and finitely-many $\vec{n}$-s, gives the complexity for the discretized and compactified theory of the complex scalar, see eq. (3.6) and the text above it. Recovering the continuum limit and decompactified space amounts to taking $\delta \rightarrow$ 0 and $L \rightarrow \infty$ respectively. The latter is implemented by replacing sums with integrals: ${ }^{23}$

$$
\sum_{\vec{n}} \rightarrow \int_{-\tilde{N}}^{\tilde{N}} d^{d-1} n=\left(\frac{L}{2 \pi}\right)^{d-1} \int_{-\pi / \delta}^{\pi / \delta} d^{d-1} k
$$

where we have switched from the discrete label $\vec{n}$ to the continuous label $\vec{k}$

$$
\vec{k} \equiv \frac{2 \pi \vec{n}}{L}
$$

cf. footnote 13 , and $\tilde{N}$ was defined in eq. (3.6). In this construction we also have to replace the frequency by the continuous frequency $\omega_{k}=\sqrt{k^{2}+m^{2}}$ (see the discussion in section 5.1 of [11] for more details). Similarly, the parameter $\lambda_{n}$ in eq. (3.19) will be replaced with

$$
\lambda_{k} \equiv \frac{\omega_{k}}{\mu_{g}}=\frac{\sqrt{k^{2}+m^{2}}}{\mu_{g}} .
$$

\footnotetext{
${ }^{23}$ There appears to be a typo in (188) of [11] in that the r.h.s. is missing the prefactor we have in (4.38).
} 
Furthermore, taking the continuum limit amounts to extending the domain of integration in eq. (4.38) to be the full $\mathbb{R}^{d-1}$. As already mentioned in the introduction, the complexity in QFT has UV divergences and needs to be regularized. This was the reason why we regulated the theory on the lattice in the first place. The divergences of the complexity for the cTFD state will be the same as those for a product state constructed from two copies of the vacuum state since the UV structure is similar in these two states. When working in the continuum limit, without a lattice, it is possible to regulate the UV divergences by introducing a momentum cutoff $|\vec{k}|<\Lambda$ as the limit of integration in eq. (4.38). For our calculations however, we will focus mostly on differences of complexities which yield finite quantities and therefore we will be able to simply take $\Lambda \rightarrow \infty$.

\subsection{Complexity of formation}

Following along the lines of section 5.3 of [11], we will now investigate the complexity of formation [38] of the complex scalar field in the charged thermofield double state

$$
\Delta \mathcal{C}_{\mathrm{cTFD}}(t=0) \equiv \mathcal{C}_{\mathrm{cTFD}}(t=0)-\mathcal{C}_{\mathrm{vac}(L \otimes R)},
$$

where $\mathcal{C}_{\mathrm{vac}(L \otimes R)}$ is the complexity of two copies of the vacuum state of the same complex scalar field theory. This UV-finite quantity compares how much harder it is to prepare the cTFD state at $t=0$ compared to preparing two copies of the vacuum state in the same complex scalar theory. We will evaluate this complexity difference using equation (4.34) which provides an upper bound for the $F_{1}$ complexity. However, similarly to what was done in [11], we will everywhere assume that the values of $\lambda_{\text {ref }}$ are such that the bound (4.34) is approximately saturated (see discussion below eqs. (2.26)-(2.27)), in particular, in all our numerical analysis below, we select $\lambda_{\text {ref }}=1 .^{24}$

By setting $t=0$ to compute the complexity of formation, it is possible to analytically diagonalize the relative covariance matrix (4.31), or (4.33), of each mode. We begin by noting that at $t=0,(4.9)$ simplifies significantly:

$$
G_{\mathrm{TFD}, k}^{ \pm}(t=0, \alpha)=\operatorname{diag}\left(\lambda_{k}^{-1} e^{ \pm 2 \alpha}, \lambda_{k} e^{\mp 2 \alpha}\right),
$$

where we have appended subscripts $k$, indicating that (4.42) gives the covariance matrix of the $k$-th momentum mode, and $\lambda_{k}$ is given in eq. (4.40). Further, transforming the covariance matrix of the reference state (4.27) also to the \pm basis, we find

$$
\begin{aligned}
& G_{\mathrm{ref}, k}^{ \pm}=G_{\mathrm{ref}, k}^{L R}=\left(R_{L_{\mathbb{C}} \rightarrow L} \tilde{\oplus} R_{R_{\mathbb{C}} \rightarrow R}\right) G_{\mathrm{ref}, k}^{L R_{\mathbb{C}}}\left(R_{L_{\mathbb{C}} \rightarrow L} \tilde{\oplus} R_{R_{\mathbb{C}} \rightarrow R}\right)^{\dagger}=C \oplus C \oplus D \oplus D, \\
& C=\frac{1}{2}\left[\begin{array}{cc}
\frac{1}{\lambda_{\text {ref }}}+\frac{\lambda_{\text {ref }}}{\lambda_{k}^{2}} \frac{1}{\lambda_{\text {ref }}}-\frac{\lambda_{\text {ref }}}{\lambda_{k}^{2}} \\
\frac{1}{\lambda_{\text {ref }}}-\frac{\lambda_{\text {ref }}}{\lambda_{k}^{2}} \frac{1}{\lambda_{\text {ref }}}+\frac{\lambda_{\text {ref }}}{\lambda_{k}^{2}}
\end{array}\right], \quad D=\frac{1}{2}\left[\begin{array}{c}
\lambda_{\text {ref }}+\frac{\lambda_{k}^{2}}{\lambda_{\text {ref }}} \lambda_{\text {ref }}-\frac{\lambda_{k}^{2}}{\lambda_{\text {ref }}} \\
\lambda_{\text {ref }}-\frac{\lambda_{k}^{2}}{\lambda_{\text {ref }}} \lambda_{\text {ref }}+\frac{\lambda_{k}^{2}}{\lambda_{\text {ref }}}
\end{array}\right],
\end{aligned}
$$

where the covariance matrix here is given in the $\xi^{ \pm}$basis from eq. (4.29) and $R_{L_{\mathbb{C}} \rightarrow L}=$ $R_{R_{\mathbb{C}} \rightarrow R}$ is given by eq. (3.22) with the replacement $\lambda_{n} \rightarrow \lambda_{k}$, applied to the left and

\footnotetext{
${ }^{24}$ We keep however the general $\lambda_{\text {ref }}$ dependence in some of the analytic expressions below, since [11] have shown that there exist some other values of $\lambda_{\text {ref }}$ for which the straight line circuit provides a good approximation of the optimal circuit. A full analysis of the range of $\lambda_{\text {ref }} \neq 1$ for which the straight line circuit provides a good approximation for the optimal circuit is beyond the scope of this work.
} 
right operators, respectively. The first equality is due to the fact that the reference state covariance matrix is stationary under the change of basis $R_{L R \rightarrow \pm}$, given in (4.32). We can then obtain the relative covariance matrix in the \pm basis as follows

$$
\begin{aligned}
& \Delta_{\mathrm{cTFD}, k}^{ \pm}= \\
& {\left[G_{\mathrm{TFD}, k}^{+}\left(t_{\bar{L} R}, \alpha_{\bar{L} R}\right) \tilde{\oplus} G_{\mathrm{TFD}, k}^{+}\left(t_{L \bar{R}}, \alpha_{L \bar{R}}\right) \tilde{\oplus} G_{\mathrm{TFD}, k}^{-}\left(t_{\bar{L} R}, \alpha_{\bar{L} R}\right) \tilde{\oplus} G_{\mathrm{TFD}, k}^{-}\left(t_{L \bar{R}}, \alpha_{L \bar{R}}\right)\right]\left(G_{\mathrm{ref}, k}^{ \pm}\right)^{-1} .}
\end{aligned}
$$

As we show in appendix $\mathrm{D}$, we can in fact analytically diagonalize $\Delta_{\mathrm{cTFD}, k}$ at $t=0$.

To evaluate the complexity of formation (4.41), we must also evaluate the complexity of the vacuum, which takes a relatively simple form in the $L R_{\mathbb{C}}$ basis, in which the covariance matrices of both the vacuum and the reference state are diagonal, cf. eq. (4.27): ${ }^{25}$

$$
\begin{aligned}
G_{\text {ref }, k}^{L R_{\mathbb{C}}} & =\operatorname{diag}\left(\lambda_{\text {ref }}^{-1} \mathbb{1}_{4 \times 4}, \lambda_{\text {ref }} \mathbb{1}_{4 \times 4}\right), \\
G_{\mathrm{vac}, k}^{L R_{\mathbb{C}}} & =\operatorname{diag}\left(\lambda_{k}^{-1} \mathbb{1}_{4 \times 4}, \lambda_{k} \mathbb{1}_{4 \times 4}\right), \\
\Delta_{\mathrm{vac}, k}^{L R_{\mathbb{C}}} & =\operatorname{diag}\left(\frac{\lambda_{\text {ref }}}{\lambda_{k}} \mathbb{1}_{4 \times 4}, \frac{\lambda_{k}}{\lambda_{\text {ref }}} \mathbb{1}_{4 \times 4}\right) .
\end{aligned}
$$

Transforming (4.44) and (4.47) to the $L R$ and $L R_{\mathbb{C}}$ bases, we are then able to evaluate the complexity of formation (4.41) for each mode using the methods described in subsection 4.3, then integrate over all modes, as described in subsection 4.4, to obtain the complexity of formation for the full complex scalar cTFD.

In $[11,38]$, it was found that the complexity of formation is proportional to the entropy in several cases (see discussion in section 1 ). Therefore, $[11,38]$ have argued that it is natural to consider the ratio of complexity of formation of the TFD state over the entanglement entropy between the two sides, or equivalently the thermal entropy of the thermal state obtained on each side after tracing out the other. The entropy for the uncharged TFD is obtained from the partition function with Bose-Einstein statistics by differentiating it with respect to the temperature (cf. eqs. (201)-(202) of [11]) and reads:

$$
S_{\mathrm{TFD}}=\operatorname{vol} \int \frac{d^{d-1} k}{(2 \pi)^{d-1}}\left(\frac{\beta \omega_{k}}{e^{\beta \omega_{k}}-1}-\log \left(1-e^{-\beta \omega_{k}}\right)\right)
$$

where $\omega_{k}=\sqrt{k^{2}+m^{2}}$ and vol $=L^{d-1}$ is an IR regulator for the volume of the field theory. For the cTFD state, we are dealing with two sets of modified temperatures and times, as indicated in eqs. (4.22)-(4.23). We therefore suggest that it is natural to normalize our result with respect to the following sum of entropies

$$
S_{\mathrm{cTFD}}=S_{\mathrm{TFD}}\left(\beta \rightarrow \beta_{L \bar{R}}\right)+S_{\mathrm{TFD}}\left(\beta \rightarrow \beta_{\bar{L} R}\right)
$$

This, of course, coincides with the entanglement entropy between the $L$ and $R$ sides of the cTFD. The entanglement entropy between the two sides of the cTFD of the complex scalar

\footnotetext{
${ }^{25}$ The vacuum covariance matrix is obtained by replacing $\lambda_{\text {ref }} \rightarrow \lambda_{k}$ in the reference covariance matrix, as is clear by comparison of the physical and reference Hamiltonians (3.20) and (3.27).
} 
field can be expressed as

$$
\begin{aligned}
& S_{\mathrm{cTFD}}=\frac{\mathrm{vol}}{\beta^{d-1}}[s(\beta m, \beta \mu)+s(\beta m,-\beta \mu)] \\
& s(x, y) \equiv \frac{\Omega_{d-2}}{(2 \pi)^{d-1}} \int_{0}^{\infty} d u u^{d-2}\left[\frac{g(u, x, y)}{e^{g(u, x, y)}-1}-\log \left(1-e^{-g(u, x, y)}\right)\right]
\end{aligned}
$$

where $g$ is given by

$$
g(u, x, y) \equiv \sqrt{u^{2}+x^{2}}-y
$$

In most plots below, we will consider the ratio

$$
\frac{\Delta \mathcal{C}_{1}(|\mathrm{cTFD}(t=0)\rangle)}{S_{\mathrm{cTFD}}} .
$$

However, since at low temperatures the entropy goes to zero, in order to study the low temperature limits we will sometimes present un-normalized plots.

\subsubsection{Vanishing chemical potential}

As we shall numerically verify (see figure 1 below), taking the limit of vanishing chemical potential $\beta \mu \rightarrow 0$ in either the $L R$ or $L R_{\mathbb{C}}$ bases yields a complexity $\mathcal{C}(t=0)$ which matches with the complexity of the real scalar uncharged TFD [11], up to additional proportionality factors. In the $L R$ basis, there is an extra overall factor of 2 , which can be attributed to the fact that each complex field decomposes into two real fields. For the same reason, we see from eq. (4.50) that entropy is similarly doubled for the complex scalar. In the $L R_{\mathbb{C}}$ basis, we find that complexity instead receives a factor of $\sqrt{2}$ compared to the result in [11], which can be attributed to the fact that the straight line circuit is better aligned with the elementary gates of this basis so that the circuit can be generated with fewer gates, see detailed discussion in footnote 41 below.

\subsubsection{High temperature limit}

Taking $\beta \rightarrow 0$, the dimensionless parameters of the theory $\beta m, \beta \mu \rightarrow 0$ vanish. Hence, we expect to recover results proportional to those of the uncharged TFD for a massless scalar in eq. (206) of [11] and indeed we find

$$
\frac{\Delta \mathcal{C}_{1}(\mathrm{cTFD})}{S_{\mathrm{CTFD}}}=\frac{2^{d}-1}{d} \times\left\{\begin{array}{ll}
1 & L R \text { basis } \\
2^{-1 / 2} & L R_{\mathbb{C}} \text { basis }
\end{array} \quad(\beta m=\beta \mu=0) .\right.
$$

Recall that the relative factor of $2^{-1 / 2}$ in the $L R_{\mathbb{C}}$ basis can be attributed to the fact that the straight line circuit is better aligned with the elementary gates of this basis, as described above in subsection 4.5.1, see also footnote 41 below. 


\subsubsection{Low temperature limit}

Here, we consider the low temperature (large $\beta$ ) limit. Focusing first on the neutral case with $\mu=0$, and taking $\beta m \gg 1$, gives the uncharged TFD at low temperatures, which was already treated in [11], see eq. (208) there,

$$
\frac{\Delta \mathcal{C}_{1}(\mathrm{cTFD})}{S_{\mathrm{cTFD}}} \approx \frac{2^{(d+1) / 2} e^{\beta m / 2}}{\beta m} \times\left\{\begin{array}{ll}
1 & L R \text { basis } \\
2^{-1 / 2} & L R_{\mathbb{C}} \text { basis }
\end{array} .\right.
$$

As in (4.53), we have an extra factor of $2^{-1 / 2}$ in the $L R_{\mathbb{C}}$ basis.

Next, we consider the low temperature limit with positive chemical potential, i.e., $\beta m \gg 1$ and $\beta \mu \gg 1$, where without loss of generality we have chosen $\mu>0 .{ }^{26}$ We are able to obtain analytic expressions for the low temperature limits in the case where we further assume $\beta(m-\mu) \gg 1$. For the complexity of formation we obtain (see appendices $\mathrm{C}$ and $\mathrm{D}$ for the detailed derivation):

$$
\begin{aligned}
& \Delta \mathcal{C}_{1}(\mathrm{cTFD}) \approx \operatorname{vol} \Omega_{d-2} \cdot\left(\frac{m}{\pi^{2} \beta}\right)^{\frac{d-1}{2}} \Gamma\left(\frac{d-1}{2}\right) e^{-\beta(m-\mu) / 2}
\end{aligned}
$$

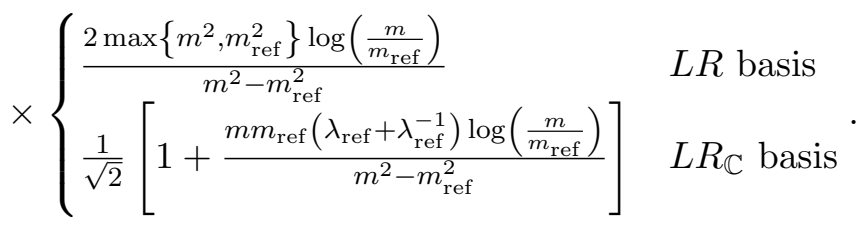

In that same limit, the entropy (4.50) of the cTFD is given by

$$
S_{\mathrm{cTFD}} \approx \operatorname{vol} \Omega_{d-2} \cdot\left(\frac{m}{2 \pi^{2} \beta}\right)^{\frac{d-1}{2}} \Gamma\left(\frac{d-1}{2}\right) e^{-\beta(m-\mu)}\left(\frac{\beta(m-\mu)}{2}\right) .
$$

Taking the ratio between complexity of formation (4.55) and entropy (4.56), we have

$$
\frac{\Delta \mathcal{C}_{1}(\mathrm{cTFD})}{S_{\mathrm{cTFD}}} \approx \frac{2^{(d-1) / 2} e^{\beta(m-\mu) / 2}}{\beta(m-\mu)}\left\{\begin{array}{ll}
\frac{4 \max \left\{m^{2}, m_{\mathrm{ref}}^{2}\right\} \log \frac{m}{m_{\mathrm{ref}}}}{m^{2}-m_{\mathrm{ref}}^{2}} & L R \text { basis } \\
\sqrt{2}\left[1+\frac{m m_{\mathrm{ref}}\left(\lambda_{\mathrm{ref}}+\lambda_{\mathrm{ref}}^{-1}\right) \log \frac{m}{m_{\mathrm{ref}}}}{m^{2}-m_{\mathrm{ref}}^{2}}\right] & L R_{\mathbb{C}} \text { basis }
\end{array} .\right.
$$

We will often be selecting $\lambda_{\text {ref }}=m_{\text {ref }} / \mu_{g}=1$, see comments around eqs. (2.26)-(2.27) and (3.28). Note that, in the low temperature limit, both the entropy and the complexity are suppressed by exponential factors, but the complexity goes to zero slower than the thermal entropy. Of course, as we go away from the large $\beta$ (low temperature) limit, this conclusion may change, as we will see in the numerics below.

\subsubsection{Numerical results}

In this section, we present numerical plots of the complexity of formation (4.41) of the complex scalar cTFD. In all the plots below, we have chosen $\lambda_{\text {ref }}=m_{\text {ref }} / \mu_{g}=1$, see related comments around eqs. (2.26)-(2.27) and (3.28). All our results here are invariant

\footnotetext{
${ }^{26}$ As noted at the end of subsection 4.2 , our results are symmetric under the change $\mu \rightarrow-\mu$.
} 


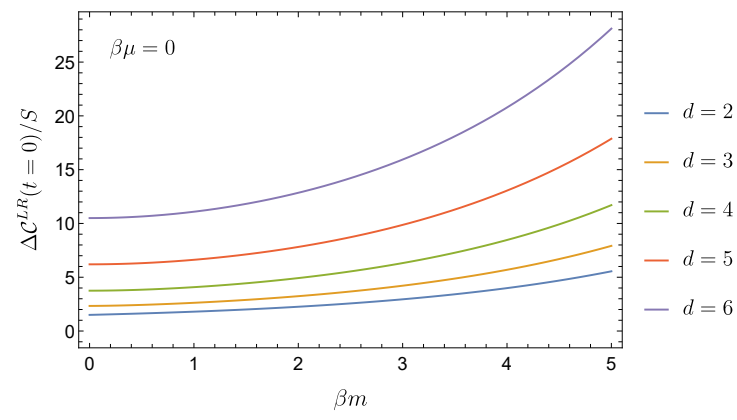

(a) $L R$ basis

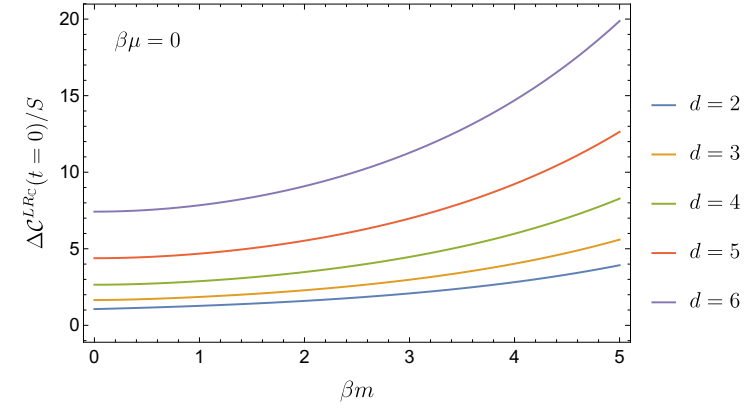

(b) $L R_{\mathbb{C}}$ basis

Figure 1. Complexity of formation, scaled by the entropy, as a function of $\beta m$, for the complex scalar cTFD in the special case $\mu=0$. Various dimensions $d$ are shown. The results for the $L R$ and $L R_{\mathbb{C}}$ bases are proportional to each other and to figure 9 in [11].

under the symmetry $\mu \rightarrow-\mu$ of the cTFD and will recover the neutral results (up to possible constants of proportionality) when $\mu=0$.

We begin with figure 1 , where we plot the case of vanishing chemical potential $\mu=0$ in both the $L R$ (figure 1a) and $L R_{\mathbb{C}}$ (figure 1b) bases. Recall that the $L R_{\mathbb{C}}$ basis was the original basis of complex operators (4.24) while the $L R$ basis is the set of operators adapted to the particle and anti-particle degrees of freedom (4.25). Note that the vanishing of $\mu$ reduces the cTFD to two uncharged TFDs. For this case, we see that the $L R$ and $L R_{\mathbb{C}}$ bases give proportional results, with a relative factor of $\sqrt{2}-$ see explanation in subsection 4.5.1 and footnote 41. In [11], the same figure (figure 9 therein) was produced for the uncharged TFD state of a real scalar field, using a basis analogous to $L R_{\mathbb{C}}$. The fact that figure $1 \mathrm{~b}$ is proportional to figure 9 of [11] provides a check of our numerics.

In figures 2 and 3 , we consider the complexity of formation for $\mu \neq 0$. We also present results for the complexity normalized by the entropy (4.50). We have added to these figures the low temperature approximations presented in subsection 4.5.3. As can be seen, moving left to right, in the right panels of subfigures $2 \mathrm{a}, 2 \mathrm{~b}, 3 \mathrm{a}$ and $3 \mathrm{~b}$, in this large $\beta$ limit, the ratio (4.52) between complexity of formation and entropy diverges exponentially for $|\mu|<m$ and appears to vary relatively slowly in the special case $^{27}|\mu|=m$. The corresponding left panels show that, for all $|\mu|<m$, the complexity becomes smaller in the low temperature limit; on the other hand, for the case $|\mu|=m$, it appears to increase. The latter case best resembles what happens in holography, where the complexity

\footnotetext{
${ }^{27}$ In the limiting case $|\mu| \rightarrow m$, the zero mode in either $\bar{L} R$ or $L \bar{R}$ becomes infinitely populated due to an unbounded effective temperature, cf. eqs. (4.22)-(4.23). However, as explained in footnote 8, in sufficiently high dimensions $d$, the full state of the scalar field may nonetheless be normalizable and have a finite particle number density. Indeed, in $d \geq 3$, the suppression by the low frequency density of states is sufficient to render the entropy integrals (4.48)-(4.50) finite as $|\mu| \rightarrow m$, which is reflective of our earlier claim that the grand canonical potential remains finite in the $|\mu| \rightarrow m$ limit for $d \geq 3$, at least when neglecting condensation. To have a physically reasonable $|\mu| \rightarrow m$ state with finite particle density would further require $d \geq 4$. In general, condensation in the zero mode $k=0$ may occur as $|\mu| \rightarrow m$, but we shall assume the condensate makes a negligible contribution such that the integral approximation (4.38) is exact in the $L \rightarrow \infty$ limit.
} 

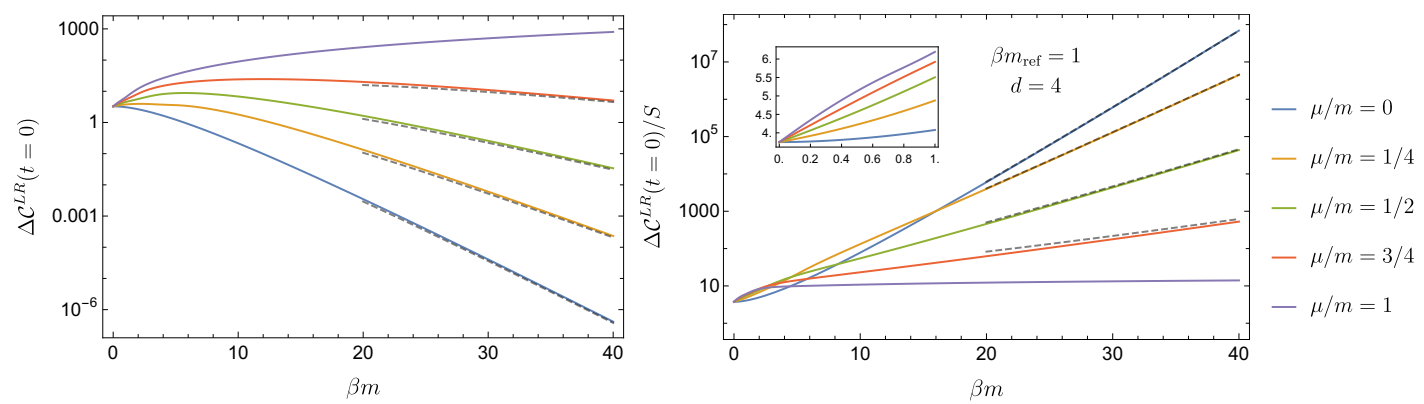

(a) Fixed $\beta m_{\text {ref }}$ and various $\mu / m$; complexity versus $\beta m$.
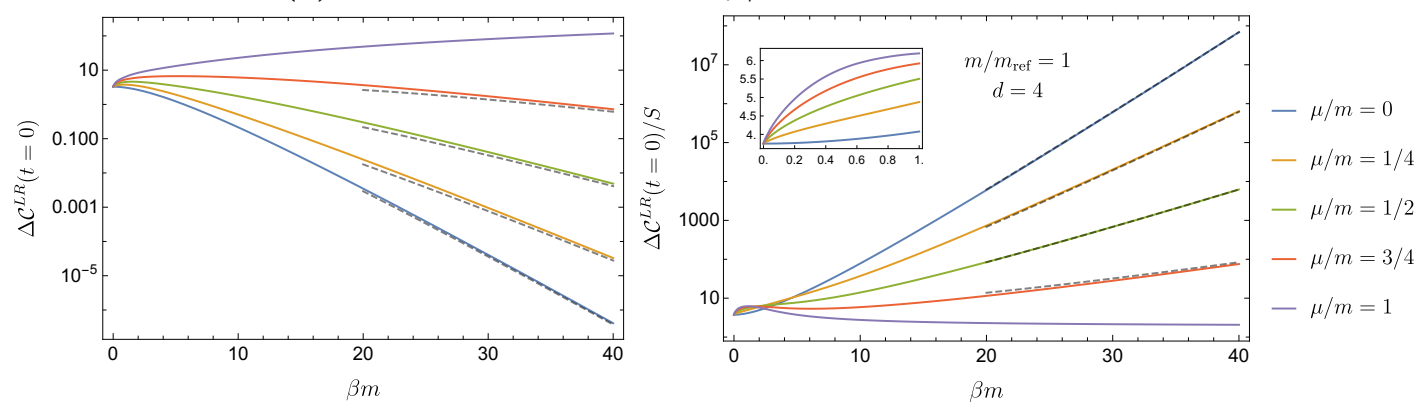

(b) Fixed $m / m_{\text {ref }}$ and various $\mu / m$; complexity versus $\beta m$.

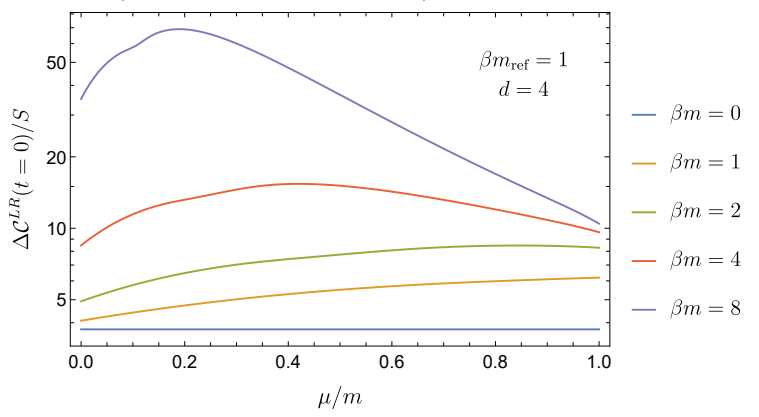

(c) Fixed $\beta m_{\text {ref }}$ and various $\beta m$; complexity versus $\mu / m$.

Figure 2. Complexity of formation in the $L R$ basis for the complex scalar cTFD in $d=4$. The curves are plotted as functions of $\beta m$ and $\mu / m$ for various fixed values of $\mu / m$ and $\beta m$, respectively (recall that $|\mu| \leq m$ ). The dashed curves in subfigures $2 \mathrm{a}$ and $2 \mathrm{~b}$ mark the low temperature limits given in subsection 4.5.3. The conformal neutral limit is obtained at the left-most points in subfigures $2 \mathrm{a}$ and $2 \mathrm{~b}$ since keeping $\mu / m$ fixed and sending $m \rightarrow 0$ means that we are also decreasing the chemical potential. We see that, in this case, the dependence on the ratio $\mu / m$ disappears and all the curves approach the same point (alternatively, this can be seen as a large temperature limit, where the chemical potential becomes negligible).

diverges in the low temperature limit at finite chemical potential, a phenomena known as the "third law of complexity" [39]. In the scalar theory, we see that this effect is not reproduced for $|\mu|<m$, as noted above and can also be seen from eq. (4.55). In general, moving from lower to higher curves in the left panels, we observe that the unnormalized complexity of formation increases as $|\mu| / m$ increases for fixed values of $\beta m$ and $\beta m_{\text {ref. Some }}$ intuition for the behaviour of the complexity of formation as a function of the chemical 

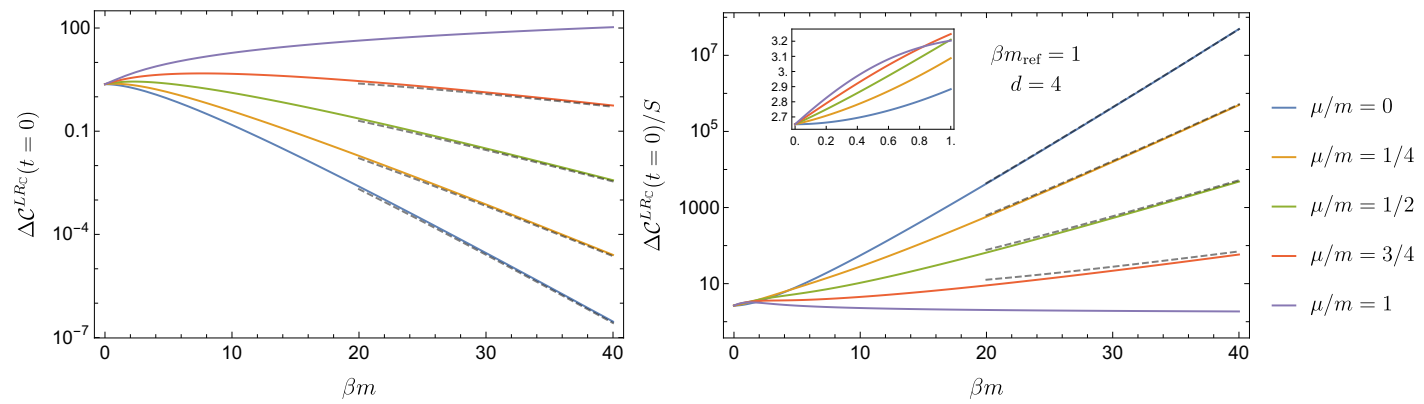

(a) Fixed $\beta m_{\text {ref }}$ and various $\mu / m$.
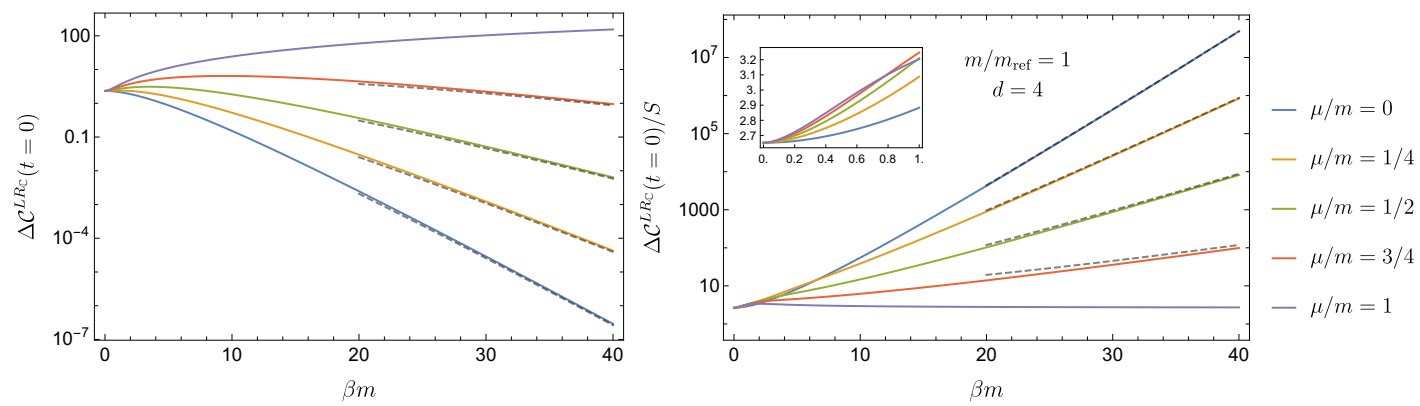

(b) Fixed $m / m_{\text {ref }}$, and various $\mu / m$.

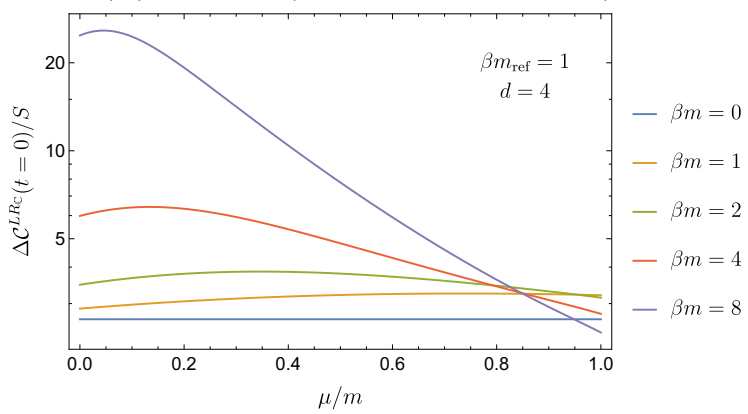

(c) Fixed $\beta m_{\text {ref }}$, and various $\beta m$.

Figure 3. Complexity of formation in the $L R_{\mathbb{C}}$ basis for the complex scalar cTFD in $d=4$. The curves are plotted as functions of $\beta m$ and $\mu / m$ for various fixed values of $\mu / m$ and $\beta m$, respectively. The dashed curves in subfigures $3 \mathrm{a}$ and $3 \mathrm{~b}$ mark the low temperature limits given in subsection 4.5.3. Note that although subfigure $3 \mathrm{a}$ fixes $\beta m_{\text {ref }}$ while subfigure $3 \mathrm{~b}$ fixes $m / m_{\text {ref }}$, the two figures are nearly identical - this is because, as shown below in figure $4 \mathrm{~b}$, the dependence on $\beta m_{\text {ref }}$ in the $L R_{\mathbb{C}}$ basis is very weak.

potential and temperature can be developed by considering the particle number density, which also increases with the temperature and chemical potential. Although the detailed dependence does not precisely match complexity (as to be expected), this perhaps suggests that states become more complex with increasing particle density. We leave it for future work to explore this correlation in greater detail. Figure 4 explores the dependence of the complexity of formation on the reference scale $\beta m_{\text {ref }}$ for general $|\mu| \leq m$. This dependence is weaker in the $L R_{\mathbb{C}}$ basis than in the $L R$ basis. We observe an approximate symmetry $\beta m_{\text {ref }} \rightarrow \varkappa /\left(\beta m_{\text {ref }}\right)$ for some constant $\varkappa$ in these figures. 


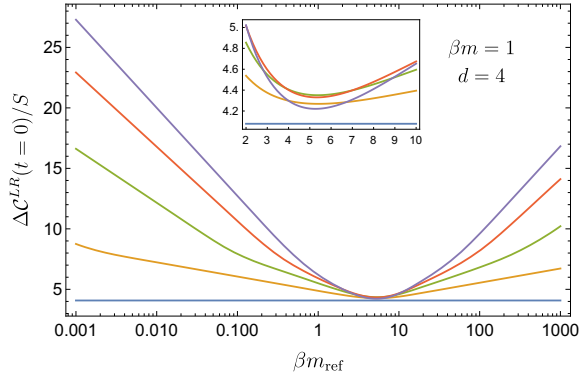

(a) $L R$ basis.

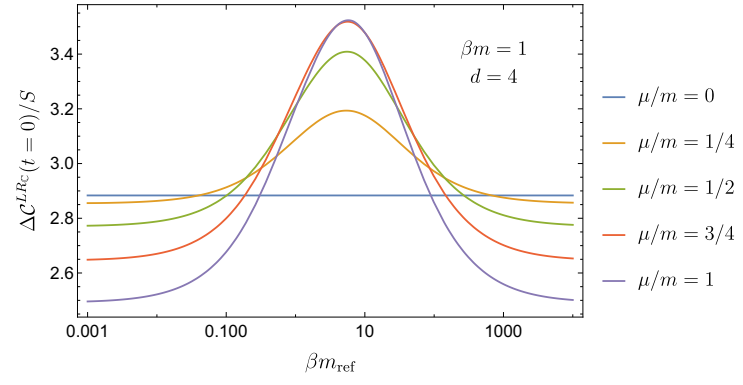

(b) $L R_{\mathbb{C}}$ basis.

Figure 4. Complexity of formation, scaled by entropy, as a function of the reference scale $\beta m_{\text {ref }}$, for the complex scalar cTFD with $\beta m=1$ in $d=4$. Various values of $\beta \mu$ are shown. The complexity of formation is independent of the reference scale when $\beta \mu=0$ as found in [11]. With non-vanishing $\mu$, the dependence is weaker in the $L R_{\mathbb{C}}$ basis than in the $L R$ basis. Note that these figures obey an approximate symmetry $\beta m_{\text {ref }} \rightarrow \varkappa /\left(\beta m_{\text {ref }}\right)$ for some constant $\varkappa$.

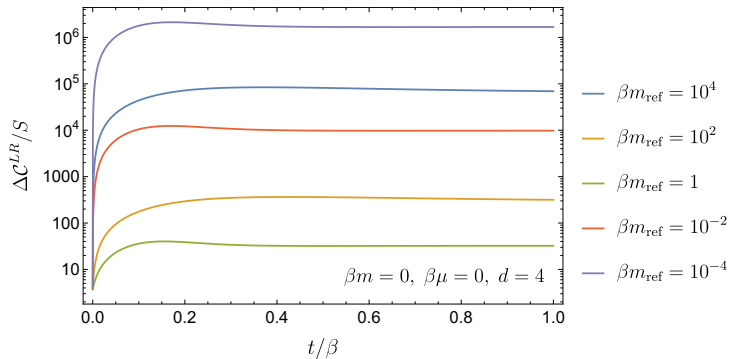

(a) $L R$ basis.

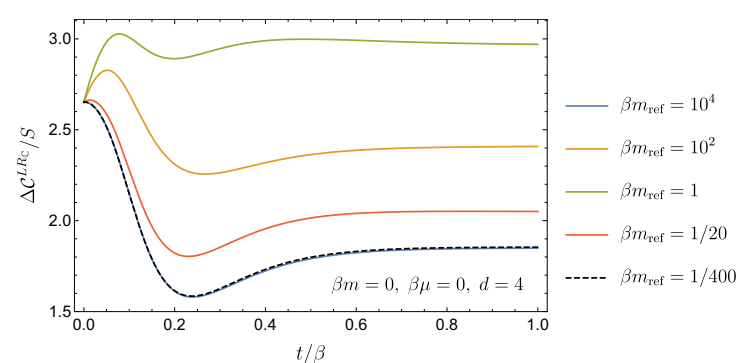

(b) $L R_{\mathbb{C}}$ basis.

Figure 5. Time dependence of the complexity, scaled by the entropy, for the complex scalar cTFD in $d=4$ with $\beta m=\beta \mu=0$. The curves for various fixed values of $\beta m_{\text {ref }}$ are plotted as functions of $t / \beta$.

\subsection{Time dependence}

Next, we consider the time dependence of complexity for the complex scalar cTFD. To calculate complexity at arbitrary times, we integrate eq. (4.34) over all modes, cf. eq. (4.38). At general times, it is cumbersome to write analytic expressions for these relative covariance matrices, so we immediately resort to numerics. We plot the complexity against time in the neutral limit (for both the $L R$ and the $L R_{\mathbb{C}}$ bases) in figure 5 and for general $|\mu| \leq m$ in the $L R$ basis in figures 6-7 and in the $L R_{\mathbb{C}}$ basis in figures 8-9.

Starting with the neutral $\mu=0$ limit in figure 5 , we observe that the complexity in the $L R$ basis (figure 5a) does not recover the results for the time dependence of the uncharged TFD of a real scalar given in [11]. In particular, note that the late time complexity becomes arbitrarily large as $\beta m_{\text {ref }}$ is taken to be very small or very large. This discrepancy with [11] is due to the fact that the ' $L R$ ' basis used for the real scalar there is more analogous to the $L R_{\mathbb{C}}$ basis of the original complex operators here. On the other hand, the complexity evaluated in the $L R_{\mathbb{C}}$ basis (figure $5 \mathrm{~b}$ ) recovers $\sqrt{2}$ times the time dependence of the 


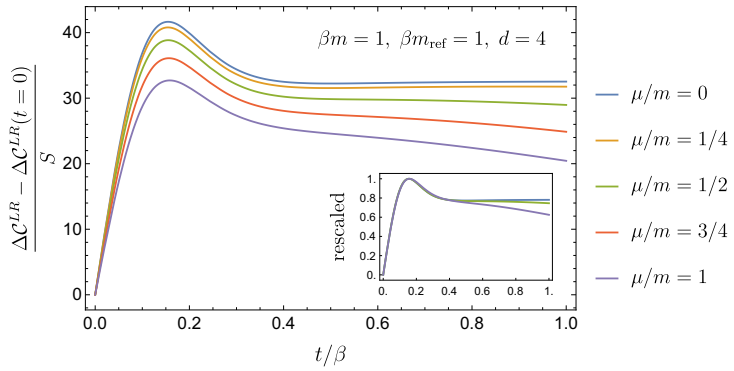

(a) $\beta m=1$.

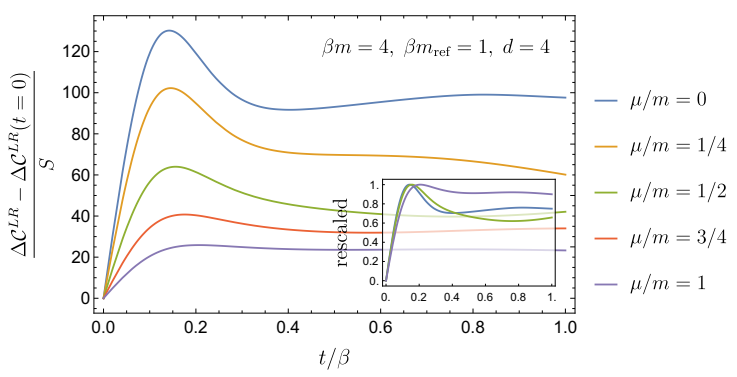

(c) $\beta m=4$.

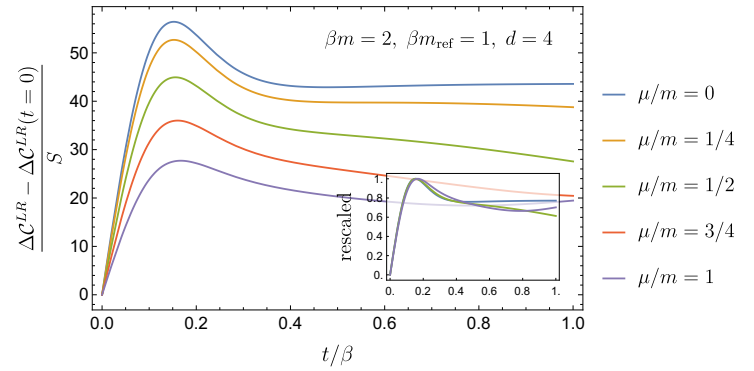

(b) $\beta m=2$.

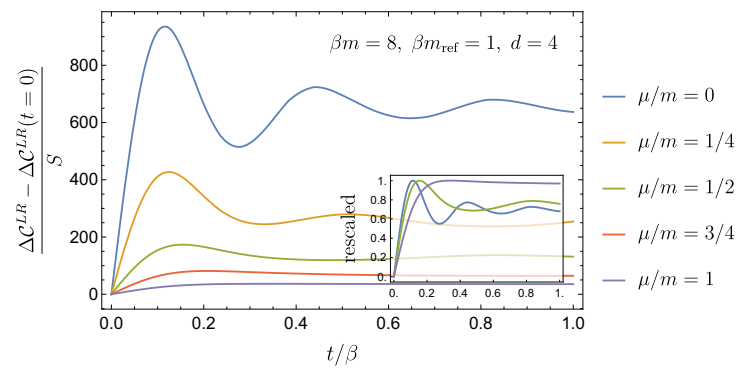

(d) $\beta m=8$.

Figure 6. Time dependence of the complexity in the $L R$ basis, scaled by the entropy, for the complex scalar cTFD in $d=4$. The subfigures correspond to $\beta m_{\mathrm{ref}}=1$ with different fixed values of $\beta m$. Curves for different fixed values of $\mu / m$ are plotted as functions of $t / \beta$.

complexity of one uncharged $\mathrm{TFD},{ }^{28}$ as given in the ' $L R$ ' basis of [11]. In particular, we see that taking extreme values of $\beta m_{\text {ref }}$ gives a finite limit for the complexity at all times in the $L R_{\mathbb{C}}$ basis.

In figures 6-7 and 8-9, we plot the time dependence of the complexity in the $L R$ and $L R_{\mathbb{C}}$ bases respectively for general values of $|\mu| \leq m$ and for various values of $\beta m>0$. Moving between the subfigures corresponding to different $\beta m$, we see that the complexity develops oscillations with a frequency proportional to $m$. This is naively to be expected: in order for the integral over single-mode (vacuum-subtracted) complexities to be convergent, the contribution of high-frequency modes must necessarily be suppressed. Hence, we expect the oscillations of the total (vacuum-subtracted) complexity to result from modes of low frequency, which are bounded from below by $\omega_{k=0}=m$.

In general, we note that, in the $L R$ basis, the complexity plotted in figures $5 \mathrm{a}$ and $6-7$ always initially increases and peaks at a global maximum, never drops below its initial value, and always saturates to a value fairly close to its global maximum. In the $L R_{\mathbb{C}}$ basis, on the other hand, the complexity does not typically stay above its initial value for all times and indeed sometimes saturates below its initial value, as shown in figures $5 \mathrm{~b}$ and $8-9$, in contrast to holographic complexity.

\footnotetext{
${ }^{28}$ We have consistently used $d=4$ across all our time-dependence plots. We have, however, separately verified that the $d=2$ analogue of the right panel of figure 5, upon subtracting off the initial value, matches figure 16 of [11] with an additional factor of $\frac{1}{\sqrt{2}}$, see subsection 4.5 .1 and footnote 41 . Recall that the entropy of the complex scalar here is double that of the real scalar in [11].
} 

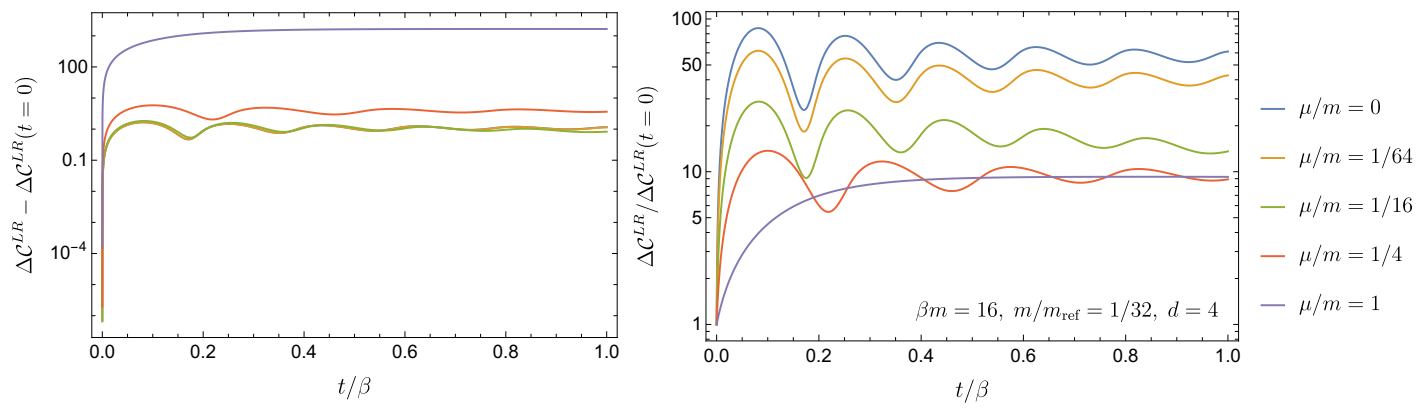

(a) $\beta m=16, m / m_{\text {ref }}=1 / 32$.
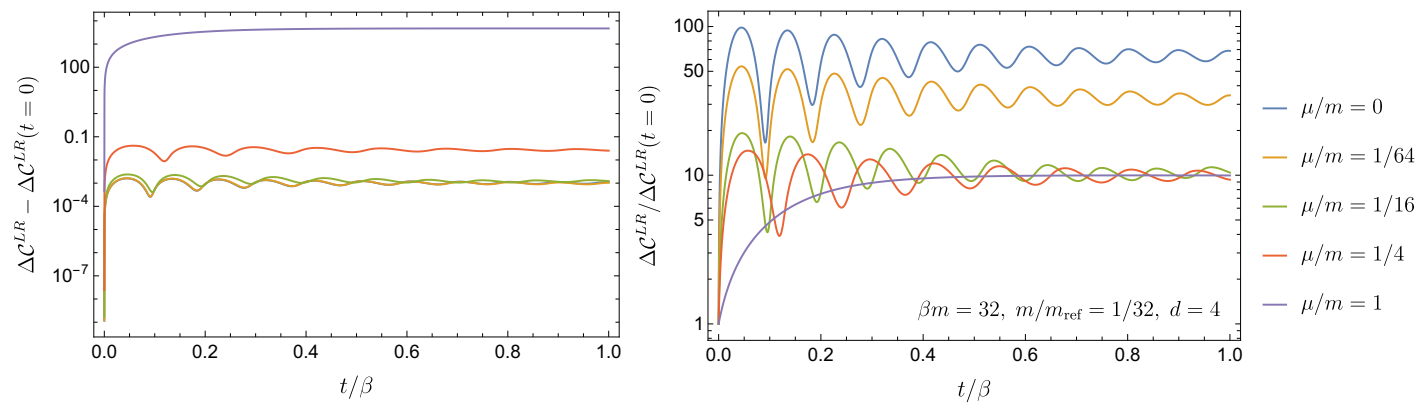

(b) $\beta m=32, m / m_{\text {ref }}=1 / 32$.

Figure 7. Oscillatory time dependence of the complexity in the $L R$ basis for the complex scalar cTFD in $d=4$ for large fixed values of $\beta m$ and even larger fixed values of $\beta m_{\text {ref. }}$. Curves for different fixed $\mu / m$ are plotted as functions of $t / \beta$. Note that the vertical axes and values of $\mu / m$ here differ from those in figure 6.

Similarly to what was found for the uncharged TFD in [11], we observe that the time dependence of complexity deviates significantly compared to the results in the holographic systems. In fact, the complexity for our Gaussian cTFD exhibits damped oscillations around some final value after times of the order of the inverse temperature. This is perhaps not surprising, since the free systems we consider here are not chaotic.

In the left panels of figures 7, 8 and 9, we observe that, in both bases, the time dependence of the (unnormalized) complexity decreases in the low temperature limit (moving between subfigures), ${ }^{29}$ keeping all the other parameters fixed, for all $|\mu|<m$. This effect is similar to the one observed in holography where the rate of computation comes to a halt as the temperature decreases. A precise comparison of the rate at which computation stops with the decrease of temperature is numerically challenging and we leave it for the future. Further, moving between the curves in each of the right panels, we observe that

\footnotetext{
${ }^{29}$ We expect that this will be the case for values of $\beta m$ larger than the value for which the unnormalized complexity of formation in the left panels of figures 2-3 starts decreasing. For instance, going from the left panel of figure $8 \mathrm{a}$ to the left panel of $8 \mathrm{~b}$, we see that only the blue, yellow and green curves appear to decrease as $\beta m$ goes from 2 to 8, cf. figure 3a. Moreover, the change in complexity compared to its initial value in the limiting $m=\mu$ case does not appear to decrease with temperature, as shown by the top curves in the left panels of figures 7 and 8 , as well as the right panels of figure 9 (this last case was not included in the left plot because of the very different orders of magnitude and it has to be rescaled with a factor of $\Delta \mathcal{C}^{L R_{\mathbb{C}}}(t=0)$, cf. the left panel of figure $3 \mathrm{~b}$, which increases with $\left.\beta m\right)$.
} 

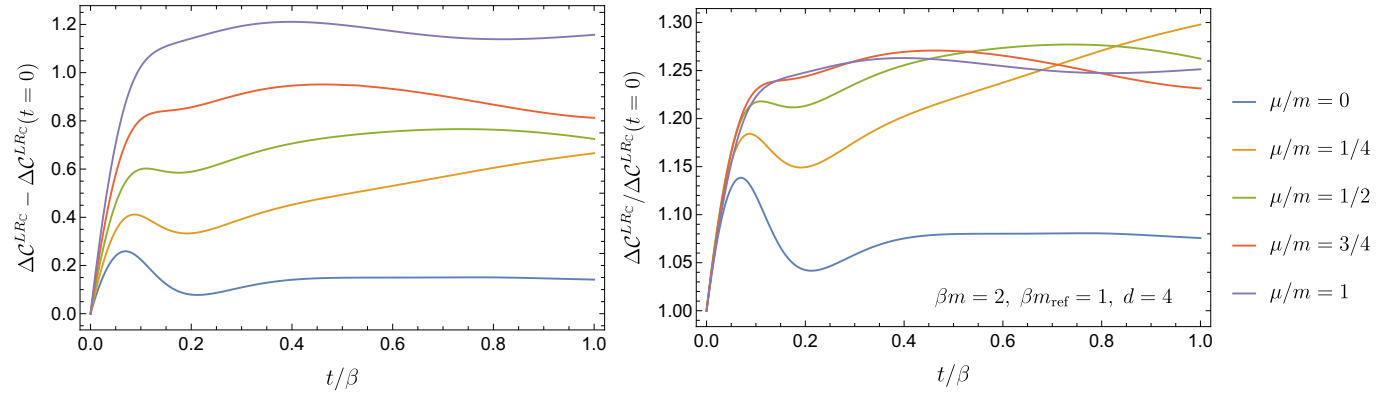

(a) $\beta m=2$.
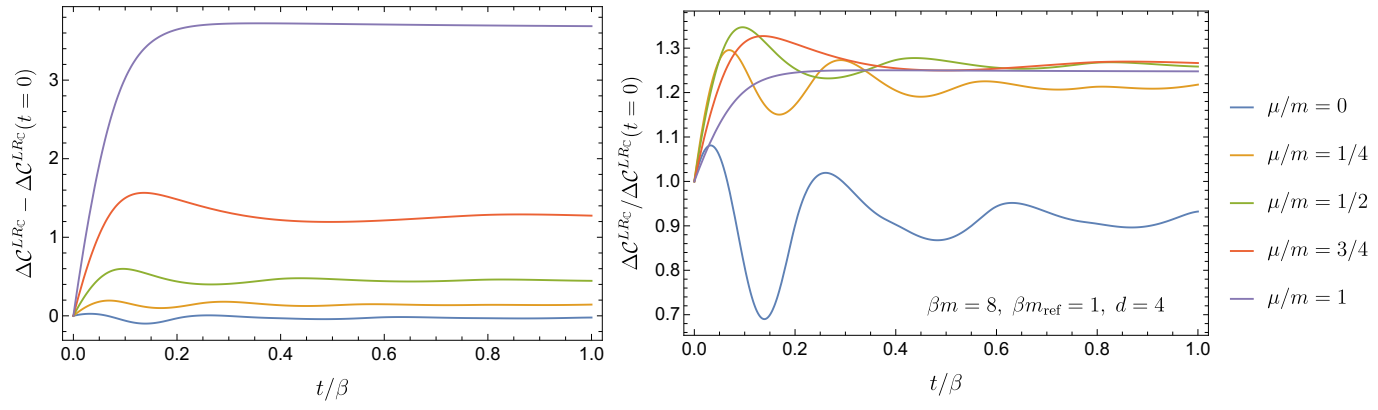

(b) $\beta m=8$.

Figure 8. Time dependence of the complexity in the $L R_{\mathbb{C}}$ basis for the complex scalar cTFD in $d=4$ with different fixed values of $\beta m$ and with $\beta m_{\text {ref }}=1$. Curves within each plot correspond to different fixed values of $\mu / m$ and are plotted as functions of $t / \beta$.
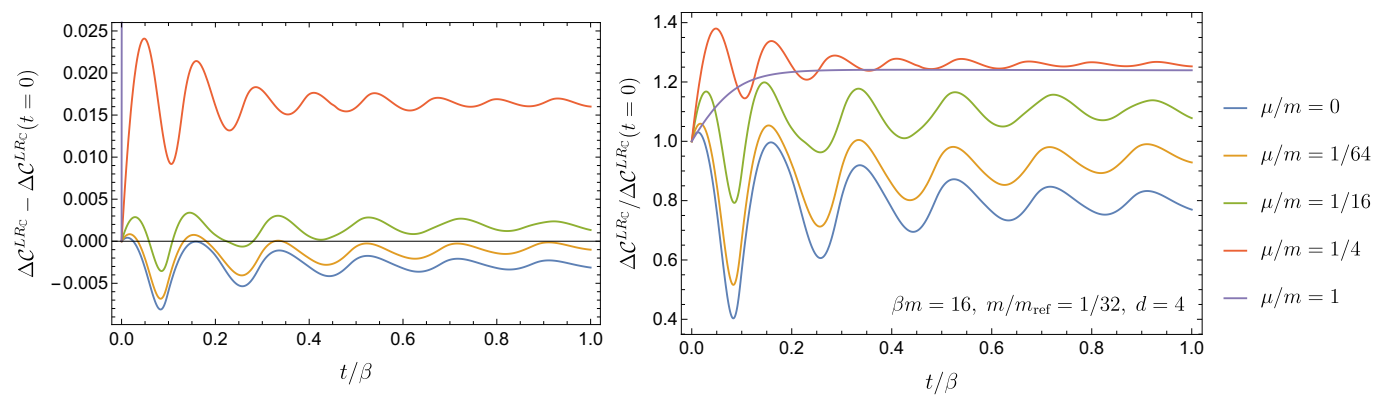

(a) $\beta m=16, m / m_{\text {ref }}=1 / 32$.
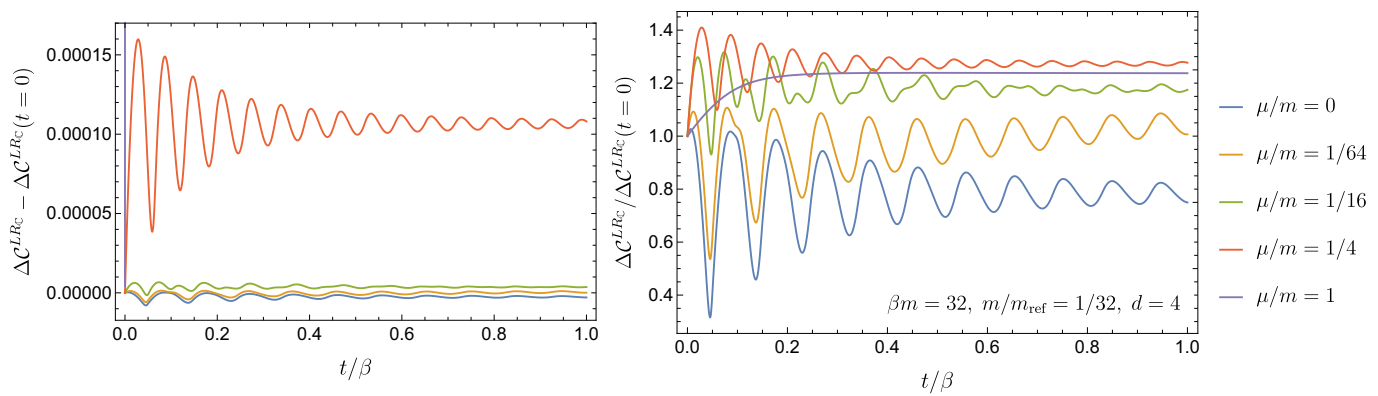

(b) $\beta m=32, m / m_{\text {ref }}=1 / 32$.

Figure 9. Oscillatory time dependence of the complexity in the $L R_{\mathbb{C}}$ basis for the cTFD in $d=4$ for large $\beta m$ and even larger $\beta m_{\text {ref. }}$. Curves for different fixed values of $\mu / m$ are plotted as functions of $t / \beta$. Note that the vertical axes and values of $\mu / m$ here differ from those in figure 8 . 
the amplitude of the fluctuations in complexity as a function of time, scaled by the initial complexity, increase as the chemical potential decreases.

\section{Comparison with holographic results}

As discussed in section 1, there are several conjectures for the gravitational dual of complexity in holography and it is expected that the qualitative comparison of free field theory results to those obtained from gravity will provide hints toward a good definition of complexity on the field theory side, applicable to the dual field theory of holographic systems. Therefore, in this section, we perform such a comparison. We start by reviewing known holographic results for the complexity of cTFDs for QFTs with holographic duals. We then compare those to our results in the previous section.

In holography, the cTFD state is dual to a charged eternal black hole, see, e.g., $[29,31,32,50]$. The complexity in this background was studied in holography in $[23,39]$ and we provide here a brief summary of those results.

The complexity of formation in the limit of small chemical potential $\mu / T \ll 1$ was studied for four boundary spacetime dimensions and was found to obey ${ }^{30}$

$$
\Delta \mathcal{C}_{A}=S\left(c_{1}+c_{2}\left(\frac{\mu}{T}\right)^{3} \ln \left(\frac{\mu}{T}\right)+\ldots\right), \quad \Delta \mathcal{C}_{V}=S\left(\tilde{c}_{1}+\tilde{c}_{2}\left(\frac{\mu}{T}\right)^{2}+\ldots\right)
$$

where $c_{1,2}$ and $\tilde{c}_{1,2}$ are order one positive constants, $S$ is the thermal entropy of each side (or the entanglement entropy between the two copies) and the subscripts $A$ and $V$ refer to the CA and CV proposals (see section 1), respectively. These expressions reproduce the neutral results (obtained for neutral eternal black holes) in the limit $\mu \rightarrow 0$. While this statement may seem obvious, checking it in holography is non-trivial, since neutral and charged eternal black holes have very different causal structures.

Furthermore, the complexity of formation of charged eternal black holes was found to be divergent in the opposite limit when the temperature is sent to zero, i.e., for $T / \mu \ll 1$ (using both the $\mathrm{CV}$ and the CA proposals) ${ }^{31}$

$$
\Delta \mathcal{C}_{A, V} \sim S \ln \left(\frac{\mu}{T}\right)+\ldots
$$

\footnotetext{
${ }^{30}$ In the result for the CA proposal, we have neglected a term of order $(\mu / T)^{3}$. The coefficient of this term was found in [39] to depend on the normalization constant $\alpha$ of the null-normals to the boundaries of the WDW patch. Our recent understanding, however, is that one should include a counter term [51] to remove this parametrization-dependence on the null-normals of the WDW patch. It would be interesting to examine the effect of this addition. In the CV result, we have neglected a term of order $(\mu / T)^{4}$. In the setup of [39], the chemical potential was assumed to be positive; however, since the definition of the cTFD state is invariant under changing $\mu \rightarrow-\mu$ as well as interchanging the left and right sides, we know that the result for negative chemical potential should take the same form with $\mu \rightarrow|\mu|$ in all the equations below.

${ }^{31}$ In these expressions, we have neglected a constant term. For the case of the CA complexity, this constant term depends on the normalization constant $\alpha$ of the null normals. It would be interesting to examine the effect of including the counter term restoring reparametrization invariance [51] on this constant. Note that this result is not valid for the case of spherical black holes with chemical potential below a certain critical value. In this case, for a sufficiently small chemical potential, pure AdS with a background gauge field is recovered in the zero temperature limit rather than an extremal black hole. As a consequence, the entanglement entropy and the complexity of formation, using both CV and CA, vanish.
} 
where again the proportionality coefficients in these relations are order one positive constants. This curious behaviour is a result of an IR divergence due to the infinitely long throat of the wormhole in the extremal (zero-temperature) limit. The interpretation suggested in [39] was that of a "third law of complexity", 32 namely that cTFD states at finite chemical potential and zero temperature are infinitely more complex compared to their finite temperature counterparts and cannot be formed by any physical process during a finite amount of time. Note that $S$ remains finite as $T \rightarrow 0$ in the holographic setup.

A number of results concerning the time dependence of complexity for charged eternal black holes are also available [23, 39]. Just like for neutral eternal black holes, also here the complexity exhibits a linear increase at late times. The authors of [23] have proposed that the late time rate of change in the CA complexity should obey a modified version of Lloyd's bound

$$
\frac{d \mathcal{C}_{A}}{d t} \leq \frac{2}{\pi}\left[(M-\mu C)-\left.(M-\mu C)\right|_{\mathrm{gs}}\right],
$$

where $M$ is the black hole mass and $C$ is its charge and where the subscript ground state (gs) indicates the thermodynamic quantities associated to the state minimizing $(M-\mu C)$ for a given value of the chemical potential. ${ }^{33}$ It was found that large or intermediate charged black holes ${ }^{34}$ violate this bound at late times, while small black holes only exhibit smaller violations in the approach to late times. In any event, the time evolution of complexity for charged black holes was found to behave more regularly than the neutral time evolution using the CA proposal. It was also found to approach the correct uncharged limit using both the $\mathrm{CA}$ and $\mathrm{CV}$ proposals. Furthermore, using both $\mathrm{CV}$ and $\mathrm{CA}$, holographic complexity was found to grow quadratically $\mathcal{C} \sim t^{2}$ at early times due to the time-reversal symmetry of the charged black hole geometry. ${ }^{35}$ Finally, the rate of change in complexity was found to vanish at all times in the extremal (vanishing temperature) limit using both the $\mathrm{CV}$ and the CA complexity proposals.

Our analysis of the cTFD in the previous section resulted in the following features which can be tested for consistency and qualitatively compared to the holographic results. First, all our results obey the symmetry $\mu \rightarrow-\mu$ of the cTFD state. Furthermore, for the complexity of formation in both the $L R$ and $L R_{\mathbb{C}}$ bases as well as for time evolution in the $L R_{\mathbb{C}}$ basis, we were able to demonstrate that the neutral results are recovered upon setting the chemical potential to zero, as is the case for holographic systems. On the other hand, we found that the complexity of formation increases with the chemical potential, see figures 2a, 2b, 3a and 3b. This is unlike what happens in holography, cf. figure 26 in [39].

\footnotetext{
${ }^{32}$ Note that the third law of thermodynamics takes a slightly different form for the free theory compared to the holographic system; the value of the entropy approached at zero temperature is zero for the free theory, while the entropy approaches a finite constant for the zero temperature limit of the holographic system.

${ }^{33}$ For the case of spherical black holes with small chemical potential, this is the vacuum state with a constant gauge field while, for spherical black holes of larger the chemical potential or planar and hyperbolic block holes, this is an extremal black hole at the same value of $\mu[23,39]$.

${ }^{34}$ I.e. charged black holes whose horizon radius is much larger, or of the same order as the AdS scale.

${ }^{35}$ The charged case smoothly interpolates to the neutral case, where CA interestingly gives a neighbourhood around $t=0$ where the rate of change in holographic complexity is exactly zero, then discontinuously becomes infinitely negative, only to quickly grow to a positive value in a short time [39].
} 
Figures 2a, 2b, 3a and 3b, further supported by an analytic low-temperature expansion, also demonstrate that only in the special case $m=|\mu|$ do we possibly have a "third law of complexity" for our simple scalar system - that is, when $m=|\mu|$, the complexity of formation appears to diverge with decreasing temperature. Note that, in the case of the complex scalar, we have to keep the relation $|\mu| \leq m$ and hence we never really approach the conformal limit at fixed chemical potential, which would be the most relevant limit for comparison with holography.

We further evaluated the time dependence of complexity and observed that the maximal (and average) change from the original value of the complexity increases with the chemical potential. Furthermore, we observe that this change decreases as the temperature decreases (except possibly for the limiting case $|\mu|=m$ ). This can be seen from figures 7 and 9. This effect is similar to the holographic observation that the rate of change in complexity vanishes when we approach the zero-temperature limit, i.e., the computation comes to a halt in this limit.

Similarly to previous works in the neutral case [11], we find that the time dependence of complexity deviates significantly compared to the results in the holographic systems. This is perhaps not surprising, since the free systems we consider do not enjoy the chaotic behaviour expected of holographic systems. Specifically, we find that complexity exhibits rapid changes followed by damped oscillations after a time of order the inverse temperature $\beta$, and seems to converge towards some final value either below or above the initial complexity. Furthermore, we find that the frequency of the damped oscillations scales approximately linearly with the field mass $m$.

It is easily verified (at least numerically) that the complexity of the complex scalar cTFD presented here is time-reversal-invariant, as in the holographic complexity conjectures. However, the complexity of the complex scalar cTFD does not have a vanishing rate of change in the early time limit; rather, it appears to vary linearly as $\sim|t|$, as in the uncharged case [11]. We would like to suggest that the contrast between this and the quadratic growth of holographic complexity may be due to the orientation of the basis of elementary gates chosen for evaluating the $F_{1}$ complexity.

For visual simplicity, let us consider a subset of elementary gates which mutually commute. Naively considering these gates alone, the $F_{1}$ complexity may be visualized as measuring lengths along grid lines denoting the action of these gates - see figure 10. In this picture, states of fixed $F_{1}$ complexity relative to the reference state live on a 'diamond' centered on the reference state with faces ${ }^{36}$ running diagonally with respect to the grid. Our result $\mathcal{C}_{1}-\mathcal{C}_{1}(t=0) \sim|t|$ then suggests that we have chosen elementary gates, so that the initial time evolution of the complex scalar cTFD has a component orthogonal to such a diamond. In particular, requiring 'smooth' time evolution and time-reversal invariance around $t=0$, this analysis would seem to require a trajectory of the cTFD under time evolution to intersect the diamond in the non-tangential manner shown by the red curve of figure 10. On the other hand, the initial quadratic growth of holographic complexity $\mathcal{C}_{1}-\mathcal{C}_{1}(t=0) \sim t^{2}$ (if it is indeed identified with an $F_{1}$ type complexity) seems to indicate

\footnotetext{
${ }^{36}$ In the case illustrated in figure 10 with two commuting elementary gates which act non-trivially on the reference state, the "faces" of the diamond are one-dimensional segments.
} 


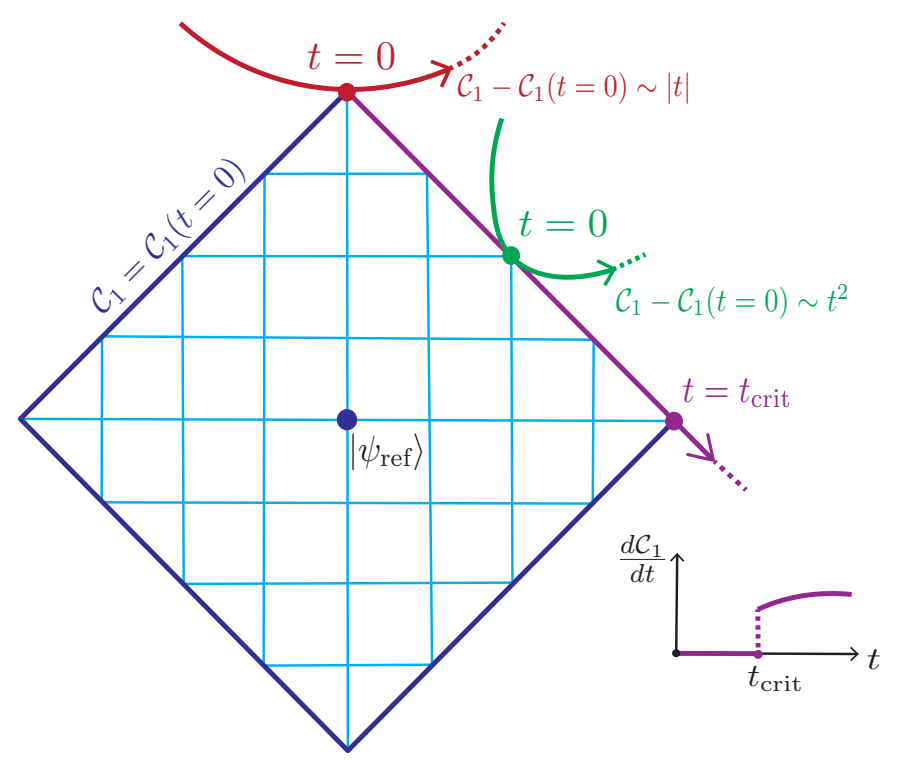

Figure 10. Illustration of the $\mathcal{C}_{1}$ complexity measuring distance from a reference state $\left|\psi_{\text {ref }}\right\rangle$ and various trajectories of target states under time evolution. (While points in this figure correspond to states, the notion of "space" here is borrowed from the circuit group manifold.) Here, the action of a set of commuting elementary gates induce motion in the north-south and east-west directions along paths shown in light blue. The dark blue diamond marks a surface of constant $\mathcal{C}_{1}$ from the reference state. Taking the target state to lie on this diamond at the initial time $t=0$, we show three possible time-reversal-invariant trajectories: a trajectory touching the diamond at $t=0$ non-tangentially, giving a $\sim|t|$ growth in complexity (red); a trajectory tangential to and touching the diamond (locally) only at $t=0$, producing a quadratic growth in complexity (green); and a trajectory which runs along a face of the diamond until a critical time $t_{\text {crit }}$ when it smoothly continues past the boundary of the face, producing a discontinuous rate of complexity growth shown in the inset plot (purple).

a time evolution that is initially tangential to the diamond, resembling the green curve. This picture may also partially provide a mechanism for the discontinuous rate of change in the CA holographic complexity of neutral black holes described by footnote 35 - see purple case in figure $10 .{ }^{37}$

While we have discussed commuting elementary gates for simplicity, we expect qualitative features of this discussion to also hold for a complete non-commuting set of elementary gates; for instance, we expect each surface of constant complexity to take the shape of a diamond with possibly warped faces and with vertices obtained by the action of single elementary gates on the reference state. It would be interesting to check whether an alternative set of gates to those chosen in this paper might be able to reproduce the quadratic growth exhibited by holographic complexity - we leave this for future work. ${ }^{38}$

\footnotetext{
${ }^{37}$ However, note that an explanation for the brief transient period where the complexity decreases, cf. footnote 35 , is still lacking.

${ }^{38}$ Note that this discussion is reminiscent of the 'first law of complexity' [52] where variations of the target state tangential to surfaces of equal complexity lead to a vanishing first order variation in complexity. In $[52,53]$, the normal to such a surface is interpreted as the 'momentum' of the optimal circuits. The leading variation of complexity is then of first (second) order in a perturbation of the target state which is parallel (orthogonal) to this momentum.
} 


\section{Summary and outlook}

In this paper, we presented a systematic framework for studying the complexity in charged system using the idea of complex phase space operators. We demonstrated how to use this machinery for the charged thermofield double state of two copies of a complex scalar field theory. We discovered that, by an appropriately chosen change of coordinates, the state can be brought to the form of a product of two uncharged thermofield double states at shifted temperatures and times. We evaluated, numerically and analytically in certain limits, the complexity of formation of the cTFD state and demonstrated that this system does not obey, in general, a third law of complexity. Hence we suggest that this rule might not hold generally outside the holographic setup. Furthermore, we presented numerical evaluations of the time dependence of the complexity and explored the effect of temperature and chemical potential. We found that the rate of change in complexity vanishes in the limit of vanishing temperature and at finite chemical potential, similarly to what happens in holographic systems. Finally, qualitatively drawing upon the early-time linear growth of complexity for the complex scalar cTFD and the quadratic growth of complexity for holographic cTFDs, we have speculated on the orientation of the early time evolution of these states relative to the elementary gates used to compute complexity.

There are a number of open questions which we leave for future studies. One direction is to conduct an analogous study of the complexity of cTFDs for fermions, which provide a setup more relevant for experimental studies. In particular, it would be interesting to explore if, in this setting, one can probe the conformal limit. Other obvious generalizations include studying the same problem using different measures of complexity, using e.g., different cost functions, the FS metric, or including penalty factors. Furthermore, it would be interesting to perform an analysis of the entanglement entropy in the cTFD state, similar to the one that was conducted in [11] for the uncharged TFD, and compare the dependence on the various parameters - size of the subregion, chemical potential and temperature to that found for holographic systems $[29,50]$. It would be interesting to explore if the factorization of each cTFD mode to two uncharged TFDs can be used to define a MERA tensor network prescription [54-56] for constructing cTFD states. In particular, one may consider combining ideas similar to those proposed in the context of the uncharged TFD state, e.g., see comments in [57] and appendix E of [38], with ideas on the incorporation of symmetries in MERA tensor networks, as in [58]. Finally, it would be interesting to evaluate the complexity in the presence of non-abelian charges.

\section{Acknowledgments}

We would like to thank Adam Chapman, Lucas Fabian Hackl, Adrian Franco-Rubio, Ben Freivogel, Timothy H. Hsieh, Rob C. Myers and Giuseppe Policastro for useful correspondence, comments and discussions. The work by HZC was done as part of the Perimeter Scholars International master's program at Perimeter Institute. Research at Perimeter Institute is supported in part by the Government of Canada through the Department of Innovation, Science and Economic Development Canada and by the Province of Ontario 
through the Ministry of Economic Development, Job Creation and Trade. SC acknowledges funding from the European Research Council (ERC) under starting grant No. 715656 (GenGeoHol) awarded to Diego M. Hofman and consolidator grant QUANTIVIOL awarded to Ben Freivogel.

\section{A Basis of $\mathfrak{s p}(2 N, \mathbb{R})$ for real/complex phase space operators}

Supplementing the discussion in section 2, we present here expressions for bases of $\mathfrak{s p}(2 N, \mathbb{R})$ used for real and complex phase space operators.

Let us begin with real phase space operators (2.2):

$$
\xi_{\mathbb{R}}=\left(q_{1}, \ldots, q_{N}, p_{1}, \ldots, p_{N}\right) .
$$

For these operators, we will use the same basis of generators as in [11]. Namely, written in terms of the operators $\hat{K}$ from eq. (2.9), we have

$$
\begin{array}{rlrl}
\hat{W}_{\mathbb{R} i j} & =\frac{1}{2}\left\{q_{i}, p_{j}\right\}, & (1 \leq i, j \leq N) \\
\hat{V}_{\mathbb{R} i j} & = \begin{cases}\frac{q_{i}^{2}}{\sqrt{2}} & \text { if } i=j \\
q_{i} q_{j} & \text { if } i<j\end{cases} \\
\hat{Z}_{\mathbb{R} i j} & = \begin{cases}\frac{p_{i}^{2}}{\sqrt{2}} & \text { if } i=j \\
p_{i} p_{j} & \text { if } i<j\end{cases} & (1 \leq i \leq j \leq N)
\end{array}
$$

where, $\{q, p\} \equiv q p+p q$ denotes symmetrization. These provide a choice of elementary gates which each contain as few operators as possible, while being Hermitian and bilinear in the operators. In terms of the $2 N \times 2 N$ matrices $k$, related to the above by eq. (2.9), we have

$$
\begin{array}{ll}
k_{a b}\left(\hat{W}_{\mathbb{R} i j}\right)=\delta_{a i} \delta_{b, j+N}+\delta_{b i} \delta_{a, j+N}, & (1 \leq i, j \leq N) \\
k_{a b}\left(\hat{V}_{\mathbb{R} i j}\right)=\left(\delta_{a i} \delta_{b j}+\delta_{b i} \delta_{a j}\right)\left\{\begin{array}{ll}
\frac{1}{\sqrt{2}} & \text { if } i=j \\
1 & \text { if } i<j
\end{array},\right. & (1 \leq i \leq j \leq N) \\
k_{a b}\left(\hat{Z}_{\mathbb{R} i j}\right)=\left(\delta_{a, i+N} \delta_{b, j+N}+\delta_{b, i+N} \delta_{a, j+N}\right)\left\{\begin{array}{ll}
\frac{1}{\sqrt{2}} & \text { if } i=j \\
1 & \text { if } i<j
\end{array} .\right. & (1 \leq i \leq j \leq N)
\end{array}
$$

Here and below, $1 \leq a, b \leq 2 N$ are matrix indices. Finally, we may multiply the above by $\Omega$, as in eq. (2.14), to obtain the corresponding $K$ :

$$
\begin{aligned}
& \left(W_{\mathbb{R} i j}\right)^{a}{ }_{b}=-\delta^{a}{ }_{i+N} \delta_{b, j+N}+\delta_{b i} \delta^{a}{ }_{j}, \quad(1 \leq i, j \leq N) \\
& \left(V_{\mathbb{R} i j}\right)^{a}{ }_{b}=-\left(\delta^{a}{ }_{i+N} \delta_{b j}+\delta_{b i} \delta^{a}{ }_{j+N}\right)\left\{\begin{array}{ll}
\frac{1}{\sqrt{2}} & \text { if } i=j \\
1 & \text { if } i<j
\end{array}, \quad(1 \leq i \leq j \leq N)\right. \\
& \left(Z_{\mathbb{R} i j}\right)^{a}{ }_{b}=\left(\delta^{a}{ }_{i} \delta_{b, j+N}+\delta_{b, i+N} \delta^{a}{ }_{j}\right)\left\{\begin{array}{ll}
\frac{1}{\sqrt{2}} & \text { if } i=j \\
1 & \text { if } i<j
\end{array} \quad \quad(1 \leq i \leq j \leq N)\right.
\end{aligned}
$$




\begin{tabular}{|c|c|c|c|c|}
\hline & $W_{\mathbb{R} q p}$ & $W_{\mathbb{R} q \bar{p}}$ & $W_{\mathbb{R} \bar{q} p}$ & $W_{\mathbb{R} \bar{q} \bar{p}}$ \\
\hline$\hat{K}$ & $\frac{q p+p q}{2}$ & $q \bar{p}$ & $\bar{q} p$ & $\frac{\bar{q} \bar{p}+\bar{p} \bar{q}}{2}$ \\
\hline$k$ & {$\left[\begin{array}{llll}0 & 0 & 1 & 0 \\
0 & 0 & 0 & 0 \\
1 & 0 & 0 & 0 \\
0 & 0 & 0 & 0\end{array}\right]$} & {$\left[\begin{array}{llll}0 & 0 & 0 & 1 \\
0 & 0 & 0 & 0 \\
0 & 0 & 0 & 0 \\
1 & 0 & 0 & 0\end{array}\right]$} & {$\left[\begin{array}{llll}0 & 0 & 0 & 0 \\
0 & 0 & 1 & 0 \\
0 & 1 & 0 & 0 \\
0 & 0 & 0 & 0\end{array}\right]$} & {$\left[\begin{array}{llll}0 & 0 & 0 & 0 \\
0 & 0 & 0 & 1 \\
0 & 0 & 0 & 0 \\
0 & 1 & 0 & 0\end{array}\right]$} \\
\hline$K$ & {$\left[\begin{array}{cccc}1 & 0 & 0 & 0 \\
0 & 0 & 0 & 0 \\
0 & 0 & -1 & 0 \\
0 & 0 & 0 & 0\end{array}\right]$} & {$\left[\begin{array}{cccc}0 & 0 & 0 & 0 \\
1 & 0 & 0 & 0 \\
0 & 0 & 0 & -1 \\
0 & 0 & 0 & 0\end{array}\right]$} & {$\left[\begin{array}{cccc}0 & 1 & 0 & 0 \\
0 & 0 & 0 & 0 \\
0 & 0 & 0 & 0 \\
0 & 0 & -1 & 0\end{array}\right]$} & {$\left[\begin{array}{cccc}0 & 0 & 0 & 0 \\
0 & 1 & 0 & 0 \\
0 & 0 & 0 & 0 \\
0 & 0 & 0 & -1\end{array}\right]$} \\
\hline
\end{tabular}

Table 1. Basis of $W$-type generators in $\mathfrak{s p}(4, \mathbb{R})$ for $\xi_{\mathbb{R}}=(q, \bar{q}, p, \bar{p})$.

\begin{tabular}{|c|c|c|c|c|c|c|}
\hline & $V_{\mathbb{R} q q}$ & $V_{\mathbb{R} q \bar{q}}$ & $V_{\mathbb{R} \bar{q} \bar{q}}$ & $Z_{\mathbb{R} p p}$ & $Z_{\mathbb{R} p \bar{p}}$ & $Z_{\mathbb{R} \bar{p} \bar{p}}$ \\
\hline$\hat{K}$ & $\frac{q^{2}}{\sqrt{2}}$ & $q \bar{q}$ & $\frac{\bar{q}^{2}}{\sqrt{2}}$ & $\frac{p^{2}}{\sqrt{2}}$ & $p \bar{p}$ & $\frac{\bar{p}^{2}}{\sqrt{2}}$ \\
\hline$k$ & {$\left[\begin{array}{cccc}\sqrt{2} & 0 & 0 & 0 \\
0 & 0 & 0 & 0 \\
0 & 0 & 0 & 0 \\
0 & 0 & 0 & 0\end{array}\right]$} & {$\left[\begin{array}{llll}0 & 1 & 0 & 0 \\
1 & 0 & 0 & 0 \\
0 & 0 & 0 & 0 \\
0 & 0 & 0 & 0\end{array}\right]$} & {$\left[\begin{array}{cccc}0 & 0 & 0 & 0 \\
0 & \sqrt{2} & 0 & 0 \\
0 & 0 & 0 & 0 \\
0 & 0 & 0 & 0\end{array}\right]$} & {$\left[\begin{array}{cccc}0 & 0 & 0 & 0 \\
0 & 0 & 0 & 0 \\
0 & 0 & \sqrt{2} & 0 \\
0 & 0 & 0 & 0\end{array}\right]$} & {$\left[\begin{array}{llll}0 & 0 & 0 & 0 \\
0 & 0 & 0 & 0 \\
0 & 0 & 0 & 1 \\
0 & 0 & 1 & 0\end{array}\right]$} & {$\left[\begin{array}{cccc}0 & 0 & 0 & 0 \\
0 & 0 & 0 & 0 \\
0 & 0 & 0 & 0 \\
0 & 0 & 0 & \sqrt{2}\end{array}\right]$} \\
\hline$K$ & {$\left[\begin{array}{ccccc}0 & 0 & 0 & 0 \\
0 & 0 & 0 & 0 \\
-\sqrt{2} & 0 & 0 & 0 \\
0 & 0 & 0 & 0\end{array}\right]$} & {$\left[\begin{array}{cccc}0 & 0 & 0 & 0 \\
0 & 0 & 0 & 0 \\
0 & -1 & 0 & 0 \\
-1 & 0 & 0 & 0\end{array}\right]$} & {$\left[\begin{array}{cccc}0 & 0 & 0 & 0 \\
0 & 0 & 0 & 0 \\
0 & 0 & 0 & 0 \\
0 & -\sqrt{2} & 0 & 0\end{array}\right]$} & {$\left[\begin{array}{cccc}0 & 0 & \sqrt{2} & 0 \\
0 & 0 & 0 & 0 \\
0 & 0 & 0 & 0 \\
0 & 0 & 0 & 0\end{array}\right]$} & {$\left[\begin{array}{llll}0 & 0 & 0 & 1 \\
0 & 0 & 1 & 0 \\
0 & 0 & 0 & 0 \\
0 & 0 & 0 & 0\end{array}\right]$} & {$\left[\begin{array}{cccc}0 & 0 & 0 & 0 \\
0 & 0 & 0 & \sqrt{2} \\
0 & 0 & 0 & 0 \\
0 & 0 & 0 & 0\end{array}\right]$} \\
\hline
\end{tabular}

Table 2. Basis of $V$ - and $Z$-type generators in $\mathfrak{s p}(4, \mathbb{R})$ for $\xi_{\mathbb{R}}=(q, \bar{q}, p, \bar{p})$.

In tables 1 and 2, we list as an example the $\hat{K}, k$, and $K$ representations of $W, V$, and $Z$ for real phase space operators in the case of $N=2$, i.e., $\mathfrak{s p}(4, \mathbb{R})$, where we have chosen $\xi_{\mathbb{R}}=(q, \bar{q}, p, \bar{p})$ as the basis of operators. In $\mathfrak{s p}(8, \mathbb{R})$, one repeats the exact same process to obtain the elementary generators written in terms of the real phase space operators in eq. (4.25):

$$
\xi^{L R} \equiv\left[q_{L}, \bar{q}_{L}, q_{R}, \bar{q}_{R}, p_{L}, \bar{p}_{L}, p_{R}, \bar{p}_{R}\right]
$$

in which we are interested in the main text.

Let us now consider the case of complex phase space operators (2.1):

$$
\xi_{\mathbb{C}}=\left(q_{1}, q_{1}^{\dagger}, \ldots, q_{N / 2}, q_{N / 2}^{\dagger}, p_{1}^{\dagger}, p_{1}, \ldots, p_{N / 2}^{\dagger}, p_{N / 2}\right)
$$

For these operators, we will use the following basis for $\mathfrak{s p}(2 N, \mathbb{R})$, written in terms of the operators $\hat{K}$ from eq. (2.9):

$$
\hat{W}_{\mathbb{C} i j}^{\ell}=\frac{1}{\sqrt{2}}\left\{\begin{array}{ll}
i\left(\left\{q_{i}^{\dagger}, p_{j}\right\}-\left\{q_{i}, p_{j}^{\dagger}\right\}\right) & \text { if } \ell=0 \\
i\left(\left\{q_{i}^{\dagger}, p_{j}^{\dagger}\right\}-\left\{q_{i}, p_{j}\right\}\right) & \text { if } \ell=1 \\
\left\{q_{i}^{\dagger}, p_{j}\right\}+\left\{q_{i}, p_{j}^{\dagger}\right\} & \text { if } \ell=2 \\
\left\{q_{i}^{\dagger}, p_{j}^{\dagger}\right\}+\left\{q_{i}, p_{j}\right\} & \text { if } \ell=3
\end{array}, \quad\left(1 \leq i, j \leq \frac{N}{2}\right)\right.
$$




$$
\begin{aligned}
& \hat{V}_{\mathbb{C} i j}^{\ell}=\left\{\begin{array}{ll}
\frac{1}{2} & \text { if } i=j \\
\frac{1}{\sqrt{2}} & \text { if } i<j
\end{array} \times\left\{\begin{array}{ll}
i\left(\left\{q_{i}^{\dagger}, q_{j}\right\}-\left\{q_{i}, q_{j}^{\dagger}\right\}\right) & \text { if } \ell=0 \text { and } i<j \\
i\left(\left\{q_{i}^{\dagger}, q_{j}^{\dagger}\right\}-\left\{q_{i}, q_{j}\right\}\right) & \text { if } \ell=1 \\
\left\{q_{i}^{\dagger}, q_{j}\right\}+\left\{q_{i}, q_{j}^{\dagger}\right\} & \text { if } \ell=2 \\
\left\{q_{i}^{\dagger}, q_{j}^{\dagger}\right\}+\left\{q_{i}, q_{j}\right\} & \text { if } \ell=3
\end{array} \quad, \quad\left(1 \leq i \leq j \leq \frac{N}{2}\right)\right.\right. \\
& \hat{Z}_{\mathbb{C}}{ }_{i j}=\left\{\begin{array}{ll}
\frac{1}{2} & \text { if } i=j \\
\frac{1}{\sqrt{2}} & \text { if } i<j
\end{array} \times\left\{\begin{array}{ll}
i\left(\left\{p_{i}^{\dagger}, p_{j}\right\}-\left\{p_{i}, p_{j}^{\dagger}\right\}\right) & \text { if } \ell=0 \text { and } i<j \\
i\left(\left\{p_{i}^{\dagger}, p_{j}^{\dagger}\right\}-\left\{p_{i}, p_{j}\right\}\right) & \text { if } \ell=1 \\
\left\{p_{i}^{\dagger}, p_{j}\right\}+\left\{p_{i}, p_{j}^{\dagger}\right\} & \text { if } \ell=2 \\
\left\{p_{i}^{\dagger}, p_{j}^{\dagger}\right\}+\left\{p_{i}, p_{j}\right\} & \text { if } \ell=3
\end{array} \quad\left(1 \leq i \leq j \leq \frac{N}{2}\right)\right.\right.
\end{aligned}
$$

These provide the simplest possible set of elementary gates ('simplest' in the sense described below eq. (A.4)). The corresponding $2 N \times 2 N$ matrices $k$, related to the above by eq. (2.9), are

$$
\begin{aligned}
& k_{a b}\left(\hat{W}_{\mathbb{C}}^{\ell}{ }_{i j}\right)=\frac{1}{\sqrt{2}}\left(i^{1-\lfloor\ell / 2\rfloor}\left[\delta_{a, 2 i-1} \delta_{b, 2 j-1+n+(\ell \bmod 2)}+\delta_{b, 2 i} \delta_{a, 2 j+n-(\ell \bmod 2)}\right]\right. \\
& \left.+(-i)^{1-\lfloor\ell / 2\rfloor}\left[\delta_{a, 2 i} \delta_{b, 2 j+n-(\ell \bmod 2)}+\delta_{b, 2 i-1} \delta_{a, 2 j-1+n+(\ell \bmod 2)}\right]\right), \\
& \left(1 \leq i, j \leq \frac{N}{2} \text { and } 0 \leq \ell \leq 3\right) \\
& k_{a b}\left(\hat{V}_{\mathbb{C} i j}^{\ell}\right)=\left\{\begin{array}{ll}
\frac{1}{2} & \text { if } i=j \\
\frac{1}{\sqrt{2}} & \text { if } i<j
\end{array} \times\left(i^{1-\lfloor\ell / 2\rfloor}\left[\delta_{a, 2 i-1} \delta_{b, 2 j-1+(\ell \bmod 2)}+\delta_{b, 2 i} \delta_{a, 2 j-(\ell \bmod 2)}\right]\right.\right. \\
& \left.+(-i)^{1-\lfloor\ell / 2\rfloor}\left[\delta_{a, 2 i} \delta_{b, 2 j-(\ell \bmod 2)}+\delta_{b, 2 i-1} \delta_{a, 2 j-1+(\ell \bmod 2)}\right]\right), \\
& \left(1 \leq i \leq j \leq \frac{N}{2} \text { and }\left\{\begin{array}{ll}
1 \leq \ell \leq 3 & \text { if } i=j \\
0 \leq \ell \leq 3 & \text { if } i<j
\end{array}\right)\right. \\
& k_{a b}\left(\hat{Z}_{\mathbb{C}}{ }_{i j}\right)=\left\{\begin{array}{ll}
\frac{1}{2} & \text { if } i=j \\
\frac{1}{\sqrt{2}} & \text { if } i<j
\end{array} \times\left(i^{1-\lfloor\ell / 2\rfloor}\left[\delta_{a, 2 i-1+n} \delta_{b, 2 j-1+n+(\ell \bmod 2)}+\delta_{b, 2 i+n} \delta_{a, 2 j+n-(\ell \bmod 2)}\right]\right.\right. \\
& \left.+(-i)^{1-\lfloor\ell / 2\rfloor}\left[\delta_{a, 2 i+n} \delta_{b, 2 j+n-(\ell \bmod 2)}+\delta_{b, 2 i-1+n} \delta_{a, 2 j-1+n+(\ell \bmod 2)}\right]\right) . \\
& \left(1 \leq i \leq j \leq \frac{N}{2} \text { and }\left\{\begin{array}{ll}
1 \leq \ell \leq 3 & \text { if } i=j \\
0 \leq \ell \leq 3 & \text { if } i<j
\end{array}\right)\right.
\end{aligned}
$$

Here, $\lfloor x\rfloor$ denotes the floor function (which rounds $x$ down to an integer) and ( $x \bmod 2$ ) means $x$ modulo 2 (taking values 0 and 1 for integer $x$ ). Finally, multiplying the above by 
$\Omega$ as in eq. (2.14), we find the corresponding matrices $K$ :

$$
\begin{aligned}
& \left(W_{\mathbb{C} i j}{ }^{\ell}{ }^{a}{ }_{b}=\frac{1}{\sqrt{2}}\left(i^{1-\lfloor\ell / 2\rfloor}\left[-\delta^{a}{ }_{2 i-1+n} \delta_{b, 2 j-1+n+(\ell \bmod 2)}+\delta_{b, 2 i} \delta^{a}{ }_{2 j-(\ell \bmod 2)}\right]\right.\right. \\
& \left.+(-i)^{1-\lfloor\ell / 2\rfloor}\left[-\delta^{a}{ }_{2 i+n} \delta_{b, 2 j+n-(\ell \bmod 2)}+\delta_{b, 2 i-1} \delta^{a}{ }_{2 j-1+(\ell \bmod 2)}\right]\right), \\
& \left(1 \leq i, j \leq \frac{N}{2} \text { and } 0 \leq \ell \leq 3\right) \\
& \left(V_{\mathbb{C} i j}{ }^{\ell}\right)_{a b}=-\left\{\begin{array}{ll}
\frac{1}{2} & \text { if } i=j \\
\frac{1}{\sqrt{2}} & \text { if } i<j
\end{array} \times\left(i^{1-\lfloor\ell / 2\rfloor}\left[\delta^{a}{ }_{2 i-1+n} \delta_{b, 2 j-1+(\ell \bmod 2)}+\delta_{b, 2 i} \delta^{a}{ }_{2 j+n-(\ell \bmod 2)}\right]\right.\right. \\
& \left.+(-i)^{1-\lfloor\ell / 2\rfloor}\left[\delta^{a}{ }_{2 i+n} \delta_{b, 2 j-(\ell \bmod 2)}+\delta_{b, 2 i-1} \delta^{a}{ }_{2 j-1+n+(\ell \bmod 2)}\right]\right), \\
& \left(1 \leq i \leq j \leq \frac{N}{2} \text { and }\left\{\begin{array}{ll}
1 \leq \ell \leq 3 & \text { if } i=j \\
0 \leq \ell \leq 3 & \text { if } i<j
\end{array}\right)\right. \\
& \left(Z_{\mathbb{C}}{ }^{\ell}{ }_{i j}\right)_{a b}=\left\{\begin{array}{ll}
\frac{1}{2} & \text { if } i=j \\
\frac{1}{\sqrt{2}} & \text { if } i<j
\end{array} \times\left(i^{1-\lfloor\ell / 2\rfloor}\left[\delta^{a}{ }_{2 i-1} \delta_{b, 2 j-1+n+(\ell \bmod 2)}+\delta_{b, 2 i+n} \delta^{a}{ }_{2 j-(\ell \bmod 2)}\right]\right.\right. \\
& \left.+(-i)^{1-\lfloor\ell / 2\rfloor}\left[\delta^{a}{ }_{2 i} \delta_{b, 2 j+n-(\ell \bmod 2)}+\delta_{b, 2 i-1+n} \delta^{a}{ }_{2 j-1+(\ell \bmod 2)}\right]\right) . \\
& \left(1 \leq i \leq j \leq \frac{N}{2} \text { and }\left\{\begin{array}{ll}
1 \leq \ell \leq 3 & \text { if } i=j \\
0 \leq \ell \leq 3 & \text { if } i<j
\end{array}\right)\right.
\end{aligned}
$$

In tables 3 and 4 , we list these generators in $\mathfrak{s p}(4, \mathbb{R})$ in the forms $\hat{K}, k$, and $K$. Note in this case that the subscripts $i, j$ above always take the value 1 and have therefore been omitted from the table. On the other hand, for the case of $\mathfrak{s p}(8, \mathbb{R})$ in which we are primarily interested in the main text, with phase space operators given by (4.24),

$$
\xi^{L R_{\mathbb{C}}} \equiv\left[\tilde{q}_{L}, \tilde{q}_{L}^{\dagger}, \tilde{q}_{R}, \tilde{q}_{R}^{\dagger}, \tilde{p}_{L}^{\dagger}, \tilde{p}_{L}, \tilde{p}_{R}^{\dagger}, \tilde{p}_{R}\right]
$$

the subscripts $i, j$ can take values 1,2 corresponding to the two sets of complex phase space operators $\left\{q_{L}, q_{L}^{\dagger}\right\}$ and $\left\{q_{R}, q_{R}^{\dagger}\right\}$ (and their conjugate momenta). The operators listed in tables 3 and 4 may then be interpreted as a subset of the full basis of $\mathfrak{s p}(8, \mathbb{R})$, upon appending subscript $L$ or $R$ to the phase space operators. All the matrix generators in this section are orthonormal with respect to the inner product (2.19).

\section{B Time evolution of the cTFD state}

The time evolution in eq. (1.1) might, at first sight, look strange due to the inclusion of the chemical potential. However, this is easily understood by coupling the uncharged TFD to an external U(1) gauge field capturing the effect of the chemical potential

$$
A_{\mu} d x^{\mu}=\mu d t
$$




\begin{tabular}{|c|c|c|c|c|}
\hline & $W_{\mathbb{C}}{ }^{0}$ & $W_{\mathbb{C}}^{1}$ & $W_{\mathbb{C}}{ }^{2}$ & $W_{\mathbb{C}}^{3}$ \\
\hline$\hat{K}$ & $\frac{i\left(-\tilde{q} \tilde{p}-\tilde{p} \tilde{q}+\tilde{q}^{\dagger} \tilde{p}^{\dagger}+\tilde{p}^{\dagger} \tilde{q}^{\dagger}\right)}{2 \sqrt{2}}$ & $\frac{i \sqrt{2}\left(-\tilde{q} \tilde{p}^{\dagger}+\tilde{q}^{\dagger} \tilde{p}\right)}{2}$ & $\frac{\tilde{q} \tilde{p}+\tilde{p} \tilde{q}+\tilde{q}^{\dagger} \tilde{p}^{\dagger}+\tilde{p}^{\dagger} \tilde{q}^{\dagger}}{2 \sqrt{2}}$ & $\frac{\sqrt{2}\left(\tilde{q}^{\dagger} \tilde{p}^{\dagger}+\tilde{q}^{\dagger} \tilde{p}\right)}{2}$ \\
\hline$k$ & $\frac{1}{\sqrt{2}}\left[\begin{array}{cccc}0 & 0 & i & 0 \\
0 & 0 & 0 & -i \\
-i & 0 & 0 & 0 \\
0 & i & 0 & 0\end{array}\right]$ & $\frac{1}{\sqrt{2}}\left[\begin{array}{cccc}0 & 0 & 0 & i \\
0 & 0 & -i & 0 \\
0 & i & 0 & 0 \\
-i & 0 & 0 & 0\end{array}\right]$ & $\frac{1}{\sqrt{2}}\left[\begin{array}{llll}0 & 0 & 1 & 0 \\
0 & 0 & 0 & 1 \\
1 & 0 & 0 & 0 \\
0 & 1 & 0 & 0\end{array}\right]$ & $\frac{1}{\sqrt{2}}\left[\begin{array}{llll}0 & 0 & 0 & 1 \\
0 & 0 & 1 & 0 \\
0 & 1 & 0 & 0 \\
1 & 0 & 0 & 0\end{array}\right]$ \\
\hline$K$ & $\frac{1}{\sqrt{2}}\left[\begin{array}{cccc}-i & 0 & 0 & 0 \\
0 & i & 0 & 0 \\
0 & 0 & -i & 0 \\
0 & 0 & 0 & i\end{array}\right]$ & $\frac{1}{\sqrt{2}}\left[\begin{array}{cccc}0 & i & 0 & 0 \\
-i & 0 & 0 & 0 \\
0 & 0 & 0 & -i \\
0 & 0 & i & 0\end{array}\right]$ & $\frac{1}{\sqrt{2}}\left[\begin{array}{cccc}1 & 0 & 0 & 0 \\
0 & 1 & 0 & 0 \\
0 & 0 & -1 & 0 \\
0 & 0 & 0 & -1\end{array}\right]$ & $\frac{1}{\sqrt{2}}\left[\begin{array}{cccc}0 & 1 & 0 & 0 \\
1 & 0 & 0 & 0 \\
0 & 0 & 0 & -1 \\
0 & 0 & -1 & 0\end{array}\right.$ \\
\hline
\end{tabular}

Table 3. Basis of $W$-type generators in $\mathfrak{s p}(4, \mathbb{R})$ for $\xi_{\mathbb{C}}=\left(q, q^{\dagger}, p^{\dagger}, p\right)$.

\begin{tabular}{|c|c|c|c|c|c|c|}
\hline & $V_{\mathbb{C}}^{1}$ & $V_{\mathbb{C}}^{2}$ & $V_{\mathbb{C}}^{3}$ & $Z_{\mathbb{C}}^{1}$ & $Z_{\mathbb{C}}^{2}$ & $Z_{\mathbb{C}}^{3}$ \\
\hline$\hat{K}$ & $\frac{i\left(-\tilde{q}^{2}+\left(\tilde{q}^{\dagger}\right)^{2}\right)}{2}$ & $\tilde{q} \tilde{q}^{\dagger}$ & $\frac{\tilde{q}^{2}+\left(\tilde{q}^{\dagger}\right)^{2}}{2}$ & $\frac{i\left(\tilde{p}^{2}-\left(\tilde{p}^{\dagger}\right)^{2}\right)}{2}$ & $\tilde{p} \tilde{p}^{\dagger}$ & $\frac{\tilde{\tilde{p}}^{2}+\left(\tilde{p}^{\dagger}\right)^{2}}{2}$ \\
\hline$k$ & {$\left[\begin{array}{cccc}0 & i & 0 & 0 \\
-i & 0 & 0 & 0 \\
0 & 0 & 0 & 0 \\
0 & 0 & 0 & 0\end{array}\right]$} & {$\left[\begin{array}{llll}1 & 0 & 0 & 0 \\
0 & 1 & 0 & 0 \\
0 & 0 & 0 & 0 \\
0 & 0 & 0 & 0\end{array}\right]$} & {$\left[\begin{array}{llll}0 & 1 & 0 & 0 \\
1 & 0 & 0 & 0 \\
0 & 0 & 0 & 0 \\
0 & 0 & 0 & 0\end{array}\right]$} & {$\left[\begin{array}{cccc}0 & 0 & 0 & 0 \\
0 & 0 & 0 & 0 \\
0 & 0 & 0 & i \\
0 & 0 & -i & 0\end{array}\right]$} & {$\left[\begin{array}{llll}0 & 0 & 0 & 0 \\
0 & 0 & 0 & 0 \\
0 & 0 & 1 & 0 \\
0 & 0 & 0 & 1\end{array}\right]$} & {$\left[\begin{array}{llll}0 & 0 & 0 & 0 \\
0 & 0 & 0 & 0 \\
0 & 0 & 0 & 1 \\
0 & 0 & 1 & 0\end{array}\right]$} \\
\hline$K$ & {$\left[\begin{array}{cccc}0 & 0 & 0 & 0 \\
0 & 0 & 0 & 0 \\
0 & -i & 0 & 0 \\
i & 0 & 0 & 0\end{array}\right]$} & {$\left[\begin{array}{cccc}0 & 0 & 0 & 0 \\
0 & 0 & 0 & 0 \\
-1 & 0 & 0 & 0 \\
0 & -1 & 0 & 0\end{array}\right]$} & {$\left[\begin{array}{cccc}0 & 0 & 0 & 0 \\
0 & 0 & 0 & 0 \\
0 & -1 & 0 & 0 \\
-1 & 0 & 0 & 0\end{array}\right]$} & {$\left[\begin{array}{cccc}0 & 0 & 0 & i \\
0 & 0 & -i & 0 \\
0 & 0 & 0 & 0 \\
0 & 0 & 0 & 0\end{array}\right]$} & {$\left[\begin{array}{llll}0 & 0 & 1 & 0 \\
0 & 0 & 0 & 1 \\
0 & 0 & 0 & 0 \\
0 & 0 & 0 & 0\end{array}\right]$} & {$\left[\begin{array}{llll}0 & 0 & 0 & 1 \\
0 & 0 & 1 & 0 \\
0 & 0 & 0 & 0 \\
0 & 0 & 0 & 0\end{array}\right]$} \\
\hline
\end{tabular}

Table 4. Basis of $V$ - and $Z$-type generators in $\mathfrak{s p}(4, \mathbb{R})$ for $\xi_{\mathbb{C}}=\left(q, q^{\dagger}, p^{\dagger}, p\right)$.

In holography, this would be interpreted as a turning on a source in the boundary field theory dual to a gauge field in the bulk. As a simple example, let us consider the effect of coupling the U(1) gauge field (B.1) to a free complex scalar field

$$
\mathscr{L}=-\left(D_{\mu} \phi\right)^{\dagger} D^{\mu} \phi-m^{2} \phi^{\dagger} \phi, \quad D_{\mu}=\partial_{\mu}-i A_{\mu},
$$

where we have set the elementary charge to one and the metric is in the mostly plus convention. Expanding out, we have

$$
\mathscr{L}=-\partial_{\mu} \phi^{\dagger} \partial^{\mu} \phi+i \mu \phi^{\dagger} \dot{\phi}-i \mu \dot{\phi}^{\dagger} \phi-\left(m^{2}-\mu^{2}\right) \phi^{\dagger} \phi,
$$

which yields the conjugate momenta

$$
\pi^{\dagger}=\frac{\partial \mathscr{L}}{\partial \dot{\phi}^{\dagger}}=\dot{\phi}-i \mu \phi, \quad \pi=\frac{\partial \mathscr{L}}{\partial \dot{\phi}}=\dot{\phi}^{\dagger}+i \mu \phi^{\dagger}
$$

and the electric charge density

$$
\mathscr{C}=i\left(\phi^{\dagger} \pi^{\dagger}-\pi \phi\right)
$$

We thus find that the effect of introducing the coupling to the $\mathrm{U}(1)$ gauge field is to deform the Hamiltonian density by $-\mu \mathscr{C}$ :

$$
\begin{aligned}
\mathscr{H} & =\dot{\phi}^{\dagger} \dot{\phi}+\vec{\nabla} \phi^{\dagger} \cdot \vec{\nabla} \phi+\left(m^{2}-\mu^{2}\right) \phi^{\dagger} \phi \\
& =\pi^{\dagger} \pi+\vec{\nabla} \phi^{\dagger} \cdot \vec{\nabla} \phi-i \mu \phi^{\dagger} \pi^{\dagger}+i \mu \pi \phi+m^{2} \phi^{\dagger} \phi \\
& =\pi^{\dagger} \pi+\vec{\nabla} \phi^{\dagger} \cdot \vec{\nabla} \phi+m^{2} \phi^{\dagger} \phi-\mu \mathscr{C} .
\end{aligned}
$$


Finally recall from eq. (1.1) that the two copies were selected to have opposite charges such that they are CPT conjugates. The time evolution in eq. (1.1) is obtained by evolving each copy according to $\mathscr{H}$ above, but with opposite values of the chemical potential, due to (once again) CPT invariance, see discussion below eq. (2.2) in [29].

\section{Derivation of the low temperature limits}

In this appendix, we present the derivation of the low temperature limits for the complexity and entropy in eqs. (4.55)-(4.57). It will be helpful in what follows to work in terms of a set of dimensionless variables $x, y, u, \bar{\gamma}$, defined by

$$
x \equiv \beta m, \quad y \equiv \beta \mu, \quad u \equiv \beta k, \quad \bar{\gamma} \equiv \frac{1}{\beta m_{\mathrm{ref}}} .
$$

In terms of these dimensionless variables, the low temperature limit is given by $x \gg 1$ and $y \gg 1$. Without loss of generality, we focus on positive chemical potentials $y>0$ (cf. comment at the end of subsection 4.2). We will see that further assuming $x-y \gg 1$ allows us to derive the low temperature limits for complexity analytically.

First, note that, for large $x, \alpha_{L \bar{R}}$, which is defined in eq. (4.8) using the modified temperature (4.23), is exponentially suppressed for all values of $u$

$$
\alpha_{L \bar{R}} \approx \exp \left\{-\frac{1}{2} g(u, x,-y)\right\} \ll 1, \quad(y \geq 0 \text { and } x \gg 1),
$$

where $g$ is defined in eq. (4.51). For $x-y \gg 1^{39}$ (e.g., when there is a finite difference between $m$ and $\mu$ while $\beta$ is large), $\alpha_{\bar{L} R}$ is also suppressed for all values of $u$

$$
\alpha_{\bar{L} R} \approx \exp \left\{-\frac{1}{2} g(u, x, y)\right\} \ll 1, \quad(x-y \gg 1) .
$$

When $y \gg 1$, the suppression in (C.2) is far stronger than the suppression in (C.3), so $\alpha_{L \bar{R}} \ll \alpha_{\bar{L} R}$. Utilizing the diagonalized form of the relative covariance matrix at $t=0$ obtained in appendix D, an analytic expression for the generator (2.27) of the straight line circuit for the cTFD can be obtained. Taking $\alpha_{L \bar{R}} \rightarrow 0$ and expanding to linear order in $\alpha_{\bar{L} R}$, we find, for a single mode of the cTFD,

$$
\begin{aligned}
& \mathcal{C}_{1}^{L R}(\mathrm{cTFD} 1 \text { mode }) \\
& \quad \approx 2\left|\log \frac{\lambda_{k}}{\lambda_{\text {ref }}}\right|+4 \alpha_{\bar{L} R} \frac{\max \left\{\lambda_{k}^{2}, \lambda_{\text {ref }}^{2}\right\}}{\lambda_{k}^{2}-\lambda_{\text {ref }}^{2}} \log \frac{\lambda_{k}}{\lambda_{\text {ref }}}, \\
& \mathcal{C}_{1}^{L R_{\mathbb{C}}}(\operatorname{cTFD} 1 \text { mode }) \\
& \quad \approx \sqrt{2}\left|\log \frac{\lambda_{k}}{\lambda_{\text {ref }}}\right|+\sqrt{2} \alpha_{\bar{L} R}\left[1+\frac{\lambda_{k}\left(1+\lambda_{\text {ref }}^{2}\right)}{\lambda_{k}^{2}-\lambda_{\text {ref }}^{2}} \log \frac{\lambda_{k}}{\lambda_{\text {ref }}}\right] .
\end{aligned}
$$

\footnotetext{
${ }^{39}$ Note that this does not necessarily imply $x \gg y$. For example $x=2 y$ can satisfy $x-y \gg 1$ but not $x \gg y$.
} 
The complexity of (two copies of) the vacuum is obtained in the limit ${ }^{40} \alpha_{\bar{L} R}, \alpha_{L \bar{R}} \rightarrow 0$ and is proportional to what was found in [11]:

$$
\mathcal{C}_{1}^{L R}(\operatorname{vac} 1 \text { mode })=2\left|\log \frac{\lambda_{k}}{\lambda_{\text {ref }}}\right|, \quad \mathcal{C}_{1}^{L R_{\mathbb{C}}}(\operatorname{vac} 1 \text { mode })=\sqrt{2}\left|\log \frac{\lambda_{k}}{\lambda_{\text {ref }}}\right|,
$$

where the proportionality factors of 2 in the $L R$ basis and $\sqrt{2}$ in the $L R_{\mathbb{C}}$ basis were explained in subsection 4.5.1. ${ }^{41}$ By subtracting these vacuum contributions, we find the complexity of formation for each mode

$$
\begin{aligned}
\Delta \mathcal{C}_{1}^{L R}(\text { cTFD } 1 \text { mode }) & \approx 4 \alpha_{\bar{L} R} \frac{\max \left\{\lambda_{k}^{2}, \lambda_{\text {ref }}^{2}\right\}}{\lambda_{k}^{2}-\lambda_{\text {ref }}^{2}} \log \frac{\lambda_{k}}{\lambda_{\text {ref }}}, \\
\Delta \mathcal{C}_{1}^{L R_{\mathbb{C}}}(\text { cTFD } 1 \text { mode }) & \approx \sqrt{2} \alpha_{\bar{L} R}\left[1+\frac{\lambda_{k}\left(1+\lambda_{\text {ref }}^{2}\right)}{\lambda_{k}^{2}-\lambda_{\text {ref }}^{2}} \log \frac{\lambda_{k}}{\lambda_{\text {ref }}}\right] .
\end{aligned}
$$

To obtain the total complexity of formation of the cTFD state, we integrate over all modes (with $u$ given by (C.1)):

$$
\Delta \mathcal{C}_{1}(\mathrm{cTFD})=\frac{\mathrm{vol}}{\beta^{d-1}} \cdot \frac{\Omega_{d-2}}{(2 \pi)^{d-1}} \int_{0}^{\infty} d u u^{d-2} \Delta \mathcal{C}_{1}(\mathrm{cTFD} 1 \text { mode })
$$

Note that the convergence of the integral is guaranteed by the exponential suppression of $\Delta \mathcal{C}_{1}$ (cTFD 1 mode) by $\alpha_{\bar{L} R}$. To continue, we apply the expansion

$$
g(u, x, y)=x-y+\frac{u^{2}}{2 x}+x \mathcal{O}\left(\frac{u^{4}}{x^{4}}\right)
$$

to $\alpha_{\bar{L} R}$ in eq. (C.3) and use it to approximate eq. (C.7). We find that, in the large $x$ limit, the suppression of $\Delta \mathcal{C}_{1}$ (cTFD 1 mode) due to $\alpha_{\bar{L} R}$ implies that the integral receives dominant contributions only when $u$ is of order at most $\vartheta \sqrt{x}$ with $\vartheta$ a large constant smaller than any positive power of $x .^{42}$ Since

$$
\lambda=\lambda_{\text {ref }} \bar{\gamma} \sqrt{u^{2}+x^{2}}
$$

\footnotetext{
${ }^{40}$ Note that, when $m>\mu$, the $\beta \rightarrow \infty$ limit of the cTFD (4.19) of each complex scalar mode reduces to the vacuum $|0,0\rangle_{L}|0,0\rangle_{R}$; in the same limit, $\alpha_{\bar{L} R}$ and $\alpha_{L \bar{R}}$ vanish, see eqs. (4.8) and (4.22)-(4.23).

${ }^{41}$ In fact, the vacuum complexity allows us to explain more explicitly what we meant in subsection 4.5.1 by saying that the relative factor of $1 / \sqrt{2}$ in the $L R_{\mathbb{C}}$ basis is due to the fact that straight line circuit is better aligned with the elementary gates of this basis; indeed, the straight line circuit for the vacuum is generated by two elementary gates, as opposed to the four used in the $L R$ basis. Specifically, in the $L R_{\mathbb{C}}$ basis, the straight line generator $\hat{K}$ for the vacuum is given by $-\frac{1}{\sqrt{2}} \log \frac{\lambda_{k}}{\lambda_{\text {ref }}}$ times the sum of the $W_{\mathbb{C}}{ }^{2}$ element in table 3 written for $L$ and $R$. In the $L R$ basis, the straight line generator $\hat{K}$ for the vacuum is $-\frac{1}{2} \log \frac{\lambda_{k}}{\lambda_{\text {ref }}}$ times the sum of the $W_{\mathbb{R} q \bar{p}}$ and the $W_{\mathbb{R} \bar{q} p}$ generators in table 1 written for both $L$ and $R$.

${ }^{42}$ This is since, for $u \gg \sqrt{x}$, the integrand becomes exponentially suppressed relative to its value for $u \lesssim \sqrt{x}$. Moreover, there is no further significant contribution in the UV, $u \gg x$, where the integrand decays exponentially as $e^{-u}$.
} 
is an approximate constant $\left.\lambda \approx \lambda\right|_{u=0}=\lambda_{\text {ref }} \bar{\gamma} x$ (with $\bar{\gamma}$ given by (C.1)) in this region, we obtain at leading order

$$
\begin{aligned}
\Delta \mathcal{C}_{1}(\mathrm{cTFD}) \approx & \frac{\operatorname{vol}}{\beta^{d-1}} \cdot \frac{\Omega_{d-2}}{(2 \pi)^{d-1}}\left(\int_{0}^{\infty} d u u^{d-2} \alpha_{\bar{L} R}\right) \\
& \times\left\{\begin{array}{ll}
\frac{4 \max \left\{(\bar{\gamma} x)^{2}, 1\right\} \log (\bar{\gamma} x)}{(\bar{\gamma} x)^{2}-1} & L R \text { basis }
\end{array} \quad(y \gg 1 \text { and } x-y \gg 1)\right. \\
\sqrt{2}\left[1+\frac{\bar{\gamma} x\left(\lambda_{\text {ref }}+\lambda_{\text {ref }}^{-1}\right) \log (\bar{\gamma} x)}{(\bar{\gamma} x)^{2}-1}\right] & L R_{\mathbb{C}} \text { basis } .
\end{aligned}
$$

We can evaluate the remaining integral using the approximation (C.3) with (C.8). The integral is simplified to a Gaussian moment:

$$
\begin{aligned}
\int_{0}^{\infty} d u u^{d-2} \alpha_{\bar{L} R} & \approx \int_{0}^{\infty} d u u^{d-2} e^{-\frac{1}{2}\left(x-y+\frac{u^{2}}{2 x}\right)} \quad(x-y \gg 1) \\
& =\frac{1}{2} \Gamma\left(\frac{d-1}{2}\right)(4 x)^{(d-1) / 2} e^{-(x-y) / 2} .
\end{aligned}
$$

Upon reverting to the dimensionful physical quantities of the theory (cf. eq. (C.1)) we obtain the result in equation (4.55).

We can apply a similar strategy to approximate the integral giving the entropy (4.50) of the cTFD. Inserting (C.8), then integrating the logarithmic term by parts, we obtain

$$
\begin{aligned}
s(x, y) & \approx \frac{\Omega_{d-2}}{(2 \pi)^{d-1}} \frac{1}{(d-1) x} \int_{0}^{\infty} d u\left(\frac{u^{d-2}}{2}\right) \frac{(d+1) u^{2}+2(d-1) x(x-y)}{e^{\frac{u^{2}}{2 x}+x-y}-1} \\
& \approx \frac{\Omega_{d-2}}{(2 \pi)^{d-1}} \begin{cases}\Gamma\left(\frac{d-1}{2}\right)(2 x)^{(d-1) / 2} e^{-(x-y)}\left(\frac{x-y}{2}\right) & \text { if } x-y \gg 1 \\
\left(\frac{1}{d-1}\right) \Gamma\left(\frac{d+3}{2}\right) \zeta\left(\frac{d+1}{2}\right)(2 x)^{(d-1) / 2} & \text { if } x=y\end{cases}
\end{aligned}
$$

where, in the case $x=y$, the integral was performed directly; and in the case $x-y \gg 1$, after selecting the leading contribution. Note that, in the $y \gg 1$ limit, $s(x, y) \gg s(x,-y)$ is the dominant term in the entropy (4.50); on the other hand, if $y=0$, then the two terms of (4.50) are equal. In the limits $y \gg 1$ and $x-y \gg 1$, we obtain (4.56) upon reverting to the dimensionful physical quantities of the theory (cf. eq. (C.1)).

Finally, taking the ratio between complexity of formation (C.10) and entropy (4.50), using the approximations (C.11) and (C.12), we have

$$
\begin{aligned}
& \frac{\Delta \mathcal{C}_{1}(\mathrm{cTFD})}{S_{\mathrm{cTFD}}} \\
& \approx \frac{2^{(d-1) / 2} e^{(x-y) / 2}}{x-y}\left\{\begin{array}{ll}
\frac{4 \max \left\{(\bar{\gamma} x)^{2}, 1\right\} \log (\bar{\gamma} x)}{(\bar{\gamma} x)^{2}-1} & L R \text { basis } \\
\sqrt{2}\left[1+\frac{\bar{\gamma} x\left(\lambda_{\mathrm{ref}}+\lambda_{\mathrm{ref}}^{-1}\right) \log (\bar{\gamma} x)}{(\bar{\gamma} x)^{2}-1}\right] & L R_{\mathbb{C}} \text { basis }
\end{array}, \quad(y \gg 1 \text { and } x-y \gg 1) .\right.
\end{aligned}
$$

This yields eq. (4.57) when using eq. (C.1) to revert to the dimensionful physical quantities of the theory. 


\section{Diagonalization of the relative covariance matrix at $t=0$}

Below, we present an additional coordinate transformation, used to derive the low temperature limits in appendix $\mathrm{C}$ and section 4.5.3. In this appendix, we have suppressed subscripts $k$ for simplicity of notation, though we still have in mind that we are treating the $k$-th momentum mode of the complex scalar (or alternatively, the cTFD of two complex harmonic oscillators). The reference and target state covariance matrices can be diagonalized by a transformation $R_{ \pm \rightarrow \Delta_{\text {cTFD }}} \in \operatorname{Sp}(4, \mathbb{R})$ to a basis which we shall call the $\Delta_{\text {cTFD }}$ basis

$$
\begin{aligned}
G_{\mathrm{ref}}^{\Delta_{\mathrm{cTFD}}}= & R_{ \pm \rightarrow \Delta_{\mathrm{cTFD}}} \cdot G_{\mathrm{ref}}^{ \pm} \cdot\left(R_{ \pm \rightarrow \Delta_{\mathrm{cTFD}}}\right)^{\dagger} \\
= & \frac{1}{4 \lambda \lambda_{\mathrm{ref}}} \operatorname{diag}\left[\left(a^{ \pm}+d^{ \pm}\right) e^{\mp 2\left(\alpha_{\bar{L} R}+\alpha_{L \bar{R}}\right)},\right. \\
& \left.\left(a^{ \pm}-d^{ \pm}\right) e^{\mp 2\left(\alpha_{\bar{L} R}+\alpha_{L \bar{R}}\right)}, a^{ \pm}-d^{ \pm}, a^{ \pm}+d^{ \pm}\right], \\
G_{\mathrm{cTFD}}^{\Delta_{\mathrm{cTFD}}=} & R_{ \pm \rightarrow \Delta_{\mathrm{cTFD}}} \cdot G_{\mathrm{cTFD}}^{ \pm} \cdot\left(R_{ \pm \rightarrow \Delta_{\mathrm{cTFD}}}\right)^{\dagger}=\mathbb{1},
\end{aligned}
$$

where ${ }^{43} G_{\mathrm{ref}}^{ \pm}$is given in eq. (4.43), $G_{\mathrm{cTFD}}^{ \pm}=G_{\mathrm{TFD}}^{ \pm}\left(t_{\bar{L} R}=0, \alpha_{\bar{L} R}\right) \tilde{\oplus} G_{\mathrm{TFD}}^{ \pm}\left(t_{L \bar{R}}=0, \alpha_{L \bar{R}}\right)$ is obtained from eq. (4.42), and

$$
\begin{aligned}
& R_{ \pm \rightarrow \Delta_{\mathrm{cTFD}}}=\left(R_{ \pm \rightarrow \Delta_{\mathrm{cTFD}}}^{(2)} \oplus R_{ \pm \rightarrow \Delta_{\mathrm{cTFD}}}^{(2)}\right) \cdot\left(G_{\mathrm{cTFD}}^{ \pm}\right)^{-1 / 2} \\
& R_{ \pm \rightarrow \Delta_{\mathrm{cTFD}}}^{(2)}=\left[\begin{array}{cc}
\cos \theta^{ \pm} & -\sin \theta^{ \pm} \\
\sin \theta^{ \pm} & \cos \theta^{ \pm}
\end{array}\right] \in \mathrm{SO}(2)
\end{aligned}
$$

with

$$
\begin{aligned}
& a^{ \pm}=\left(e^{ \pm 2 \alpha_{\bar{L} R}}+e^{ \pm 2 \alpha_{L \bar{R}}}\right)\left(\lambda^{2}+\lambda_{\text {ref }}^{2}\right), \\
& b^{ \pm}=\left(e^{ \pm 2 \alpha_{\bar{L} R}}-e^{ \pm 2 \alpha_{L \bar{R}}}\right)\left(\lambda^{2}+\lambda_{\text {ref }}^{2}\right), \\
& c^{ \pm}=2\left(\lambda^{2}-\lambda_{\text {ref }}^{2}\right) e^{ \pm\left(\alpha_{\bar{L} R}+\alpha_{L \bar{R}}\right)}, \\
& d^{ \pm}=\sqrt{\left(b^{ \pm}\right)^{2}+\left(c^{ \pm}\right)^{2}}, \\
& \theta^{ \pm}=\tan ^{-1}\left(\frac{c^{ \pm}}{b^{ \pm}-d^{ \pm}}\right) .
\end{aligned}
$$

Using eqs. (D.1)-(D.2), it is straightforward to demonstrate that we in fact have a diagonal relative covariance matrix $(2.26)$ in this basis

$$
\Delta_{\mathrm{CTFD}}^{\Delta_{\mathrm{cTFD}}}=\left(G_{\mathrm{ref}}^{\Delta_{\mathrm{cTFD}}}\right)^{-1} .
$$

Open Access. This article is distributed under the terms of the Creative Commons Attribution License (CC-BY 4.0), which permits any use, distribution and reproduction in any medium, provided the original author(s) and source are credited.

\footnotetext{
${ }^{43}$ Note that, unlike in eqs. (4.29) and (4.43), where \pm simply specifies the complete basis, here it enumerates two possibilities, i.e., $R_{+\rightarrow \Delta_{\text {cTFD }}}$ transforms the operators $\left[q_{\bar{L} R}^{+}, q_{L \bar{R}}^{+}, p_{\bar{L} R}^{+}, p_{L \bar{R}}^{+}\right]$to the new basis, while $R_{-\rightarrow \Delta_{\text {CTFD }}}$ transforms the operators $\left[q_{\bar{L} R}^{-}, q_{L \bar{R}}^{-}, p_{\bar{L} R}^{-}, p_{L \bar{R}}^{-}\right]$to the new basis. Note that the covariance matrices of these operators in the reference and cTFD states already factorize to + and - coordinate blocks $G_{\mathrm{ref}}^{+}, G_{\mathrm{ref}}^{-}$and $G_{\mathrm{cTFD}}^{+}, G_{\mathrm{cTFD}}^{-}$, see eqs. (4.28) and (4.43), and therefore it is possible to transform each of the blocks of the covariance matrices separately.
} 


\section{References}

[1] S. Aaronson, The Complexity of Quantum States and Transformations: From Quantum Money to Black Holes, 7, 2016 [arXiv: 1607.05256] [INSPIRE].

[2] M.A. Nielsen and I. Chuang, Quantum computation and quantum information, Cambridge University Press, Cambridge U.K. (2002).

[3] M.A. Nielsen, A geometric approach to quantum circuit lower bounds, quant-ph/0502070.

[4] M.A. Nielsen, M.R. Dowling, M. Gu and A.C. Doherty, Quantum computation as geometry, Science 311 (2006) 1133.

[5] M.R. Dowling and M.A. Nielsen, The geometry of quantum computation, quant-ph/0701004.

[6] R. Jefferson and R.C. Myers, Circuit complexity in quantum field theory, JHEP 10 (2017) 107 [arXiv: 1707.08570] [INSPIRE].

[7] S. Chapman, M.P. Heller, H. Marrochio and F. Pastawski, Toward a Definition of Complexity for Quantum Field Theory States, Phys. Rev. Lett. 120 (2018) 121602 [arXiv: 1707.08582] [INSPIRE].

[8] R. Khan, C. Krishnan and S. Sharma, Circuit Complexity in Fermionic Field Theory, Phys. Rev. D 98 (2018) 126001 [arXiv:1801.07620] [InSPIRE].

[9] L. Hackl and R.C. Myers, Circuit complexity for free fermions, JHEP 07 (2018) 139 [arXiv: 1803.10638] [INSPIRE].

[10] A.P. Reynolds and S.F. Ross, Complexity of the AdS Soliton, Class. Quant. Grav. 35 (2018) 095006 [arXiv:1712.03732] [INSPIRE].

[11] S. Chapman, J. Eisert, L. Hackl, M.P. Heller, R. Jefferson, H. Marrochio et al., Complexity and entanglement for thermofield double states, SciPost Phys. 6 (2019) 034 [arXiv: 1810.05151] [INSPIRE].

[12] J. Jiang and X. Liu, Circuit Complexity for Fermionic Thermofield Double states, Phys. Rev. D 99 (2019) 026011 [arXiv:1812.00193] [INSPIRE].

[13] H.A. Camargo, P. Caputa, D. Das, M.P. Heller and R. Jefferson, Complexity as a novel probe of quantum quenches: universal scalings and purifications, Phys. Rev. Lett. 122 (2019) 081601 [arXiv: 1807.07075] [INSPIRE].

[14] J. Jiang, J. Shan and J. Yang, Circuit complexity for free Fermion with a mass quench, Nucl. Phys. B 954 (2020) 114988 [arXiv:1810.00537] [INSPIRE].

[15] E. Caceres, S. Chapman, J.D. Couch, J.P. Hernández, R.C. Myers and S.-M. Ruan, Complexity of Mixed States in QFT and Holography, JHEP 03 (2020) 012 [arXiv: 1909.10557] [INSPIRE].

[16] A. Bhattacharyya, A. Shekar and A. Sinha, Circuit complexity in interacting QFTs and RG flows, JHEP 10 (2018) 140 [arXiv: 1808.03105] [INSPIRE].

[17] P. Caputa and J.M. Magan, Quantum Computation as Gravity, Phys. Rev. Lett. 122 (2019) 231302 [arXiv: 1807.04422 ] [INSPIRE].

[18] P. Caputa, N. Kundu, M. Miyaji, T. Takayanagi and K. Watanabe, Liouville Action as Path-Integral Complexity: From Continuous Tensor Networks to AdS/CFT, JHEP 11 (2017) 097 [arXiv: 1706.07056] [INSPIRE]. 
[19] A. Belin, A. Lewkowycz and G. Sárosi, Complexity and the bulk volume, a new York time story, JHEP 03 (2019) 044 [arXiv: 1811.03097] [INSPIRE].

[20] L. Susskind, Computational Complexity and Black Hole Horizons, Fortsch. Phys. 64 (2016) 24 [Addendum ibid. 64 (2016) 44] [arXiv:1403.5695] [INSPIRE].

[21] D. Stanford and L. Susskind, Complexity and Shock Wave Geometries, Phys. Rev. D 90 (2014) 126007 [arXiv:1406 . 2678] [INSPIRE].

[22] A.R. Brown, D.A. Roberts, L. Susskind, B. Swingle and Y. Zhao, Holographic Complexity Equals Bulk Action?, Phys. Rev. Lett. 116 (2016) 191301 [arXiv:1509.07876] [INSPIRE].

[23] A.R. Brown, D.A. Roberts, L. Susskind, B. Swingle and Y. Zhao, Complexity, action, and black holes, Phys. Rev. D 93 (2016) 086006 [arXiv: 1512.04993] [InSPIRE].

[24] S. Ryu and T. Takayanagi, Holographic derivation of entanglement entropy from AdS/CFT, Phys. Rev. Lett. 96 (2006) 181602 [hep-th/0603001] [INSPIRE].

[25] T. Nishioka, S. Ryu and T. Takayanagi, Holographic Entanglement Entropy: An Overview, J. Phys. A 42 (2009) 504008 [arXiv:0905.0932] [INSPIRE].

[26] D. Carmi, R.C. Myers and P. Rath, Comments on Holographic Complexity, JHEP 03 (2017) 118 [arXiv: 1612.00433] [INSPIRE].

[27] A. Reynolds and S.F. Ross, Divergences in Holographic Complexity, Class. Quant. Grav. 34 (2017) 105004 [arXiv:1612.05439] [INSPIRE].

[28] S. Chapman, H. Marrochio and R.C. Myers, Holographic complexity in Vaidya spacetimes. Part II, JHEP 06 (2018) 114 [arXiv: 1805.07262] [INSPIRE].

[29] T. Andrade, S. Fischetti, D. Marolf, S.F. Ross and M. Rozali, Entanglement and correlations near extremality: CFTs dual to Reissner-Nordström AdS $S_{5}$, JHEP 04 (2014) 023 [arXiv: 1312.2839] [INSPIRE].

[30] J.M. Maldacena, Eternal black holes in anti-de Sitter, JHEP 04 (2003) 021 [hep-th/0106112] [INSPIRE].

[31] A. Chamblin, R. Emparan, C.V. Johnson and R.C. Myers, Charged AdS black holes and catastrophic holography, Phys. Rev. D 60 (1999) 064018 [hep-th/9902170] [INSPIRE].

[32] S.A. Hartnoll, Lectures on holographic methods for condensed matter physics, Class. Quant. Grav. 26 (2009) 224002 [arXiv: 0903.3246] [INSPIRE].

[33] W. Cottrell, B. Freivogel, D.M. Hofman and S.F. Lokhande, How to Build the Thermofield Double State, JHEP 02 (2019) 058 [arXiv: 1811.11528] [inSPIRE].

[34] J. Wu and T.H. Hsieh, Variational Thermal Quantum Simulation via Thermofield Double States, Phys. Rev. Lett. 123 (2019) 220502 [arXiv:1811.11756] [INSPIRE].

[35] D. Zhu et al., Generation of thermofield double states and critical ground states with a quantum computer, Proc. Nat. Acad. Sci. 117 (2020) 25402 [arXiv:1906. 02699] [INSPIRE].

[36] J. Martyn and B. Swingle, Product Spectrum Ansatz and the Simplicity of Thermal States, Phys. Rev. A 100 (2019) 032107 [arXiv: 1812.01015] [inSPIRE].

[37] J. Maldacena and X.-L. Qi, Eternal traversable wormhole, arXiv:1804.00491 [INSPIRE].

[38] S. Chapman, H. Marrochio and R.C. Myers, Complexity of Formation in Holography, JHEP 01 (2017) 062 [arXiv:1610.08063] [INSPIRE]. 
[39] D. Carmi, S. Chapman, H. Marrochio, R.C. Myers and S. Sugishita, On the Time Dependence of Holographic Complexity, JHEP 11 (2017) 188 [arXiv:1709.10184] [INSPIRE].

[40] T. Hartman and J. Maldacena, Time Evolution of Entanglement Entropy from Black Hole Interiors, JHEP 05 (2013) 014 [arXiv: 1303.1080] [INSPIRE].

[41] L. Susskind, Entanglement is not enough, Fortsch. Phys. 64 (2016) 49 [arXiv:1411.0690] [INSPIRE].

[42] A.R. Brown, L. Susskind and Y. Zhao, Quantum Complexity and Negative Curvature, Phys. Rev. D 95 (2017) 045010 [arXiv: 1608.02612] [INSPIRE].

[43] V. Balasubramanian, M. Decross, A. Kar and O. Parrikar, Quantum Complexity of Time Evolution with Chaotic Hamiltonians, JHEP 01 (2020) 134 [arXiv:1905.05765] [INSPIRE].

[44] Y. Sekino and L. Susskind, Fast Scramblers, JHEP 10 (2008) 065 [arXiv:0808.2096] [INSPIRE].

[45] S.H. Shenker and D. Stanford, Black holes and the butterfly effect, JHEP 03 (2014) 067 [arXiv: 1306.0622] [INSPIRE].

[46] D.A. Roberts, D. Stanford and L. Susskind, Localized shocks, JHEP 03 (2015) 051 [arXiv: 1409.8180] [INSPIRE].

[47] L. Susskind and Y. Zhao, Switchbacks and the Bridge to Nowhere, arXiv:1408.2823 [INSPIRE].

[48] S. Chapman, H. Marrochio and R.C. Myers, Holographic complexity in Vaidya spacetimes. Part I, JHEP 06 (2018) 046 [arXiv: 1804.07410] [INSPIRE].

[49] M. Sinamuli and R.B. Mann, Holographic Complexity and Charged Scalar Fields, Phys. Rev. D 99 (2019) 106013 [arXiv: 1902.01912] [INSPIRE].

[50] S. Kundu and J.F. Pedraza, Aspects of Holographic Entanglement at Finite Temperature and Chemical Potential, JHEP 08 (2016) 177 [arXiv: 1602.07353] [INSPIRE].

[51] L. Lehner, R.C. Myers, E. Poisson and R.D. Sorkin, Gravitational action with null boundaries, Phys. Rev. D 94 (2016) 084046 [arXiv: 1609.00207] [InSPIRE].

[52] A. Bernamonti, F. Galli, J. Hernandez, R.C. Myers, S.-M. Ruan and J. Simón, First Law of Holographic Complexity, Phys. Rev. Lett. 123 (2019) 081601 [arXiv: 1903.04511] [InSPIRE].

[53] A. Bernamonti, F. Galli, J. Hernandez, R.C. Myers, S.-M. Ruan and J. Simón, Aspects of The First Law of Complexity, arXiv: 2002.05779 [INSPIRE].

[54] G. Vidal, Entanglement Renormalization, Phys. Rev. Lett. 99 (2007) 220405 [cond-mat/0512165] [INSPIRE].

[55] G. Vidal, Class of Quantum Many-Body States That Can Be Efficiently Simulated, Phys. Rev. Lett. 101 (2008) 110501 [quant-ph/0610099] [INSPIRE].

[56] G. Vidal, Entanglement renormalization: an introduction, arXiv:0912.1651.

[57] G. Evenbly and G. Vidal, Tensor network renormalization yields the multiscale entanglement renormalization ansatz, Phys. Rev. Lett. 115 (2015) 200401 [arXiv:1502.05385].

[58] S. Singh and G. Vidal, Symmetry protected entanglement renormalization, Phys. Rev. B 88 (2013) 121108 [arXiv:1303.6716] [INSPIRE]. 